\begin{abstract}
Title of Document:

EFFICIENT SIMULATION OF ELECTRON TRAPPING IN LASER AND PLASMA WAKEFIELD ACCELERATION
\end{abstract}

Sepehr Morshed, PhD., 2009

Directed By:

Professor Thomas M. Antonsen, Jr.

Department of Electrical and Computer

Engineering

Plasma based laser Wakefield accelerators (LWFA) have been a subject of interest in the plasma community for many years. In LWFA schemes the laser pulse must propagate several centimeters and maintain its coherence over this distance, which corresponds to many Rayleigh lengths. These Wakefields and their effect on the laser can be simulated in the quasistatic approximation. The 2D, cylindrically symmetric, quasistatic simulation code, WAKE is an efficient tool for the modeling of short-pulse laser propagation in under dense plasmas [P. Mora \& T.M. Antonsen Phys. Plasmas 4, 1997]. The quasistatic approximation, which assumes that the driver and its wakefields are undisturbed during the transit time of plasma electrons, through the pulse, cannot, however, treat electron trapping and beam loading. 
Here we modify WAKE to include the effects of electron trapping and beam loading by introducing a population of beam electrons. Background plasma electrons that are beginning to start their oscillation around the radial axis and have energy above some threshold are removed from the background plasma and promoted to "beam" electrons. The population of beam electrons which are no longer subject to the quasistatic approximation, are treated without approximation and provide their own electromagnetic field that acts upon the background plasma. The algorithm is benchmarked to OSIRIS (a standard particle in cell code) simulations which makes no quasistatic approximation. We also have done simulation and comparison of results for centimeter scale $\mathrm{GeV}$ electron accelerator experiments from LBNL. These modifications to WAKE provide a tool for simulating $\mathrm{GeV}$ laser or plasma wakefield acceleration on desktop computers. 


\title{
EFFICIENT SIMULATION OF ELECTRON TRAPPING IN LASER AND PLASMA WAKEFIELD ACCELERATION
}

\author{
By
}

Sepehr Morshed

Dissertation submitted to the Faculty of the Graduate School of the University of Maryland, College Park, in partial fulfillment of the requirements for the degree of Doctorate in Philosophy 2009

Advisory Committee:

Professor Thomas M. Antonsen, Jr., Chair

Professor Howard M. Milchberg

Professor Victor L. Granatstein

Dr. Parvez Guzdar

Professor Adil B. Hassam 
C Copyright by Sepehr Morshed 2009 


\section{Dedication}

To the loving memory of my father, Hassan Morshed. 


\section{Acknowledgements}

I would like to thank Prof. Antonsen who has guided me in every step of the way throughout my work with him. Especially during the review process of my dissertation Prof. Antonsen was most helpful in examination of my work in the timeliest manner. He has shown incredible amount of patience and understanding which I am forever grateful for them.

I also want to thank Prof. Howard Milchberg, Prof. Victor Granatstein, Dr. Parvez Guzdar and Prof. Adil Hassam for serving on my dissertation committee.

I am much in debt of gratitude to my colleague Dr. John Palastro who has helped enormously to advance my understanding of my field of research when I started in this area of work. Also I want to acknowledge my other colleague Andrew Pearson for his support.

I want to thank my mom for her unconditional support throughout my life and especially during the hard times after my father passed away. Without her encouragement and love I would not be at this point in my life. I also want to thank my brother and sister during these years of graduate school for their help.

Finally, I want to thank my wife, Sarah, whose encouragements, love and support in every step of the way paved my way to gain my degree and more importantly fall in love with her even more as we go through this journey of life. 


\section{Table of Contents}

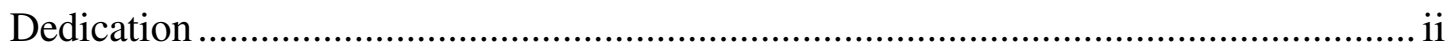

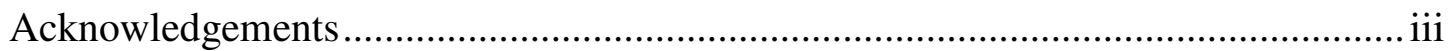

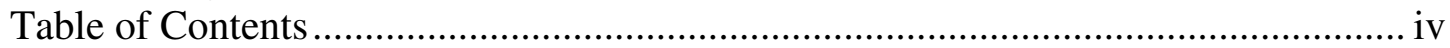

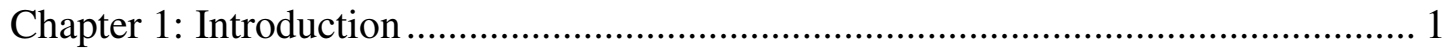

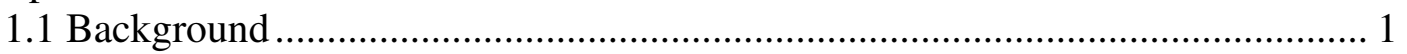

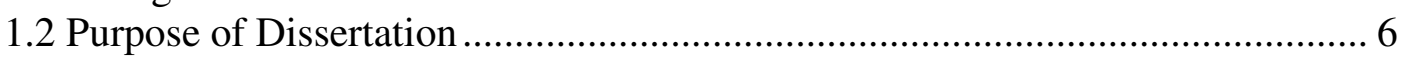

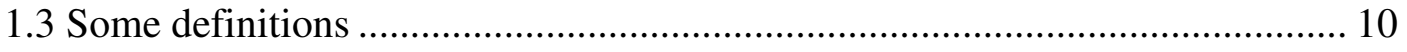

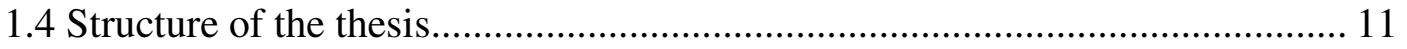

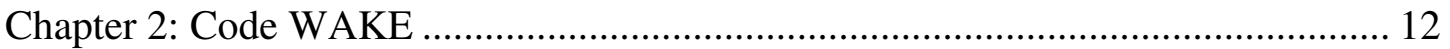

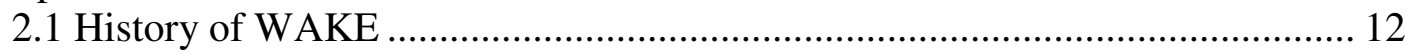

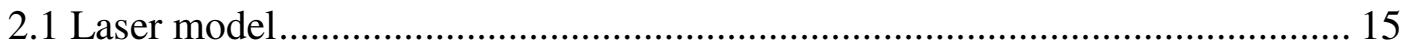

2.3 Governing equations of the code for plasma particles ....................................... 16

2.4 Equations of the code with beam particles added to the system........................ 23

2.5 Algorithm of Self-consistent beam dynamics................................................ 24

2.6 Externally injected particles simulation in WAKE and Benchmarking ........... 26

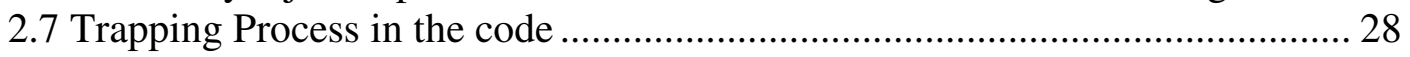

2.8 Laser Wakefield Acceleration in WAKE .................................................... 32

2.8.1 Study of particle trajectories ................................................................... 32

2.8.2 Study of the effects of selecting different thresholds................................. 34

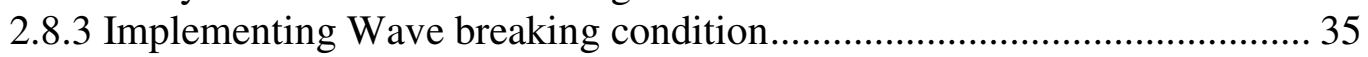

2.8.4 Simultaneous implementation of promotion conditions .............................. 36

2.9. Summary of the chapter .......................................................................... 38

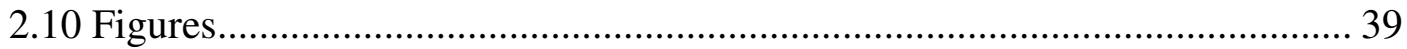

Chapter 3: Comparing WAKE to OSIRIS simulation .............................................. 59

3.1. The Conditions of OSIRIS simulation........................................................ 59

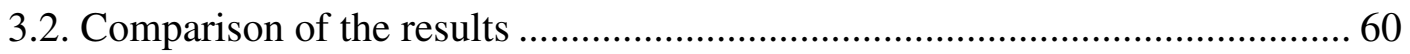

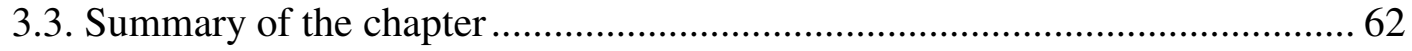

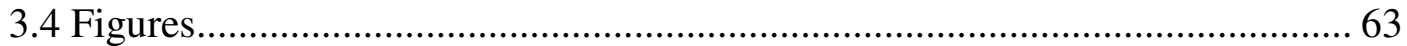

Chapter 4: Simulation of LNBL experiment for $1 \mathrm{GeV}$ electron beam........................ 67

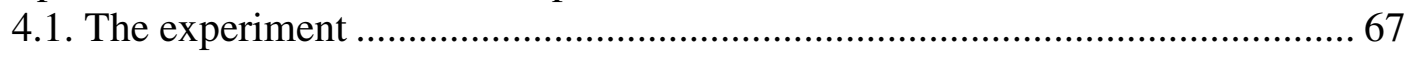

4.2. Using Externally injected particles to simulate the experiment.........................68 68

4.3. Self-trapping using only threshold trapping condition ......................................... 69

4.4 Self-trapping with implementation of all trapping conditions .......................... 71

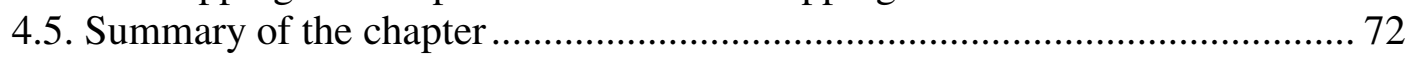

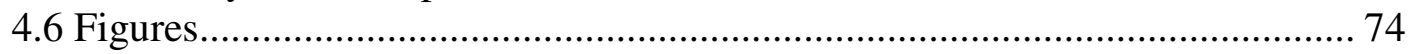

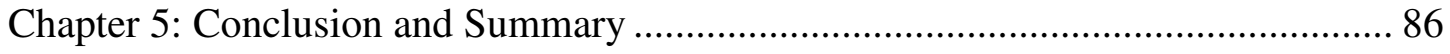

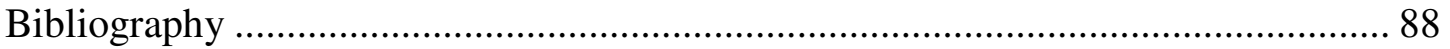




\section{Chapter 1: Introduction}

\section{$\underline{1.1 \text { Background }}$}

There have been many significant discoveries and broad applications using charged particle accelerators; from detecting new sub atomic particles to developing radiation sources in medicine, nuclear engineering, material science and more. These came directly from advances in understanding and engineering of particle accelerators. Accelerator energies have increased by an order of magnitude every decade, until the past decade for which the rate of increase has slowed down. [1]

Current accelerators use Radio Frequency (RF) fields to accelerate particles and achieve acceleration gradient in the $10-100 \mathrm{MV} / \mathrm{m}$ range. Gradients are limited mostly due to breakdown which happens on the walls of the accelerator structure. In order for us to continue the exponential growth of the attainable energies for the accelerators, there are two choices: First, continue building bigger and more expensive accelerators with the same basic technology as the old ones. Second, come up with new technologies in order to maintain the advancement in the field.

This work is focused on the later choice, specifically, simulation of Laser Wakefield Accelerators (LWFA) [5-8] which is one of the new methods in accelerating particles in the general category of plasma accelerators.

Plasma accelerators, unlike conventional accelerators which use RF waves to accelerate particles, utilize plasma oscillation waves that are excited by a laser pulse (LWFA) or driver beam either electrons or positrons (PWFA) [9-12]. It's been mentioned 
before that one of the biggest limitations in the accelerating gradient of RF accelerators is breakdown at the walls of structure. On the other hand in plasma, which is already ionized, the system can sustain orders of magnitude higher electric fields and thus higher accelerating gradients.

Nearly 30 years ago Tajima and Dawson [13] proposed using laser beams in plasma to create plasma waves for accelerating electrons. In this scheme the wake generated by the laser pulse (for the case of LWFA or electron bunch for PWFA) is traveling with phase velocity close to the group velocity of the driver and near the speed of light. Acceleration gradients on the order of $100 \mathrm{GV} / \mathrm{m}$ can be achieved, nearly three orders of magnitude higher than conventional RF structures [14],[15].

$$
E_{0}=\omega_{p} m c / e \rightarrow E_{0}[V / c m] \simeq 0.96 n_{0}^{1 / 2}\left[\mathrm{~cm}^{-3}\right]
$$

where $\omega_{p}=\left(4 \pi n_{0} e^{2} / m_{e}\right)^{1 / 2}$ is the electron plasma frequency and $n_{0}$ is the plasma electron density[16].

There are 4 basic schemes (figure 1) in plasma accelerators, which all work on the same principal: the longitudinal electric field of a relativistic traveling plasma wave is used to accelerate charged particles.

- Plasma Wake Field Accelerator(PWFA) A high energy electron bunch

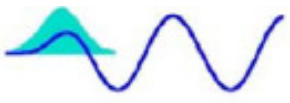

- Laser Wake Field Accelerator(LWFA)

A single short-pulse of photons

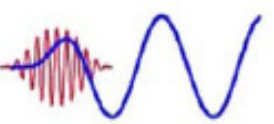

- Plasma Beat Wave Accelerator(PBWA)

Two-frequencies, i.e., a train of pulses

- Self Modulated Laser Wake Field Accelerator(SMLWFA)

Raman forward scattering instability

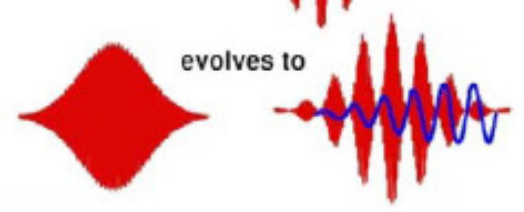


Fig.1.1) Schematic of 4 methods of plasma acceleration [17]

- Plasma Wakefield acceleration (PWFA): The plasma wave is formed by a relativistic electron or positron bunch. This beam should be terminated in times shorter than plasma period [18],[19].

- Laser Wakefield acceleration (LWFA): The new generation of compact, ultra short pulse lasers $(<1 \mathrm{ps})$ ultra intense $>10^{18}\left[\mathrm{Wcm}^{-2}\right]$, is used to generate an electron plasma wave. A pulse with the duration on the order of plasma period is used [20]-[26].

- Plasma beat-wave acceleration (PBWA): Two conventional long pulse ( 100 ps) modest intensity lasers $\left(\mathrm{I} \sim 10^{14}-10^{16}\left[\mathrm{Wcm}^{-2}\right]\right)$ are used with frequencies $\omega_{1}$ and $\omega_{2}$. The electron plasma wave arises based on the beating of the two laser pulses whose frequencies differ by the plasma frequency $\omega_{p}=\omega_{1}-\omega_{2}$. The problem with this scheme is producing plasma with a precise value electron density [27].

- Self-modulated laser Wakefield acceleration (SMLWFA): The formation of the plasma wave is achieved by a long laser pulse that becomes self modulated by the stimulated Raman forward scattering instability. [28],[29]

To create a plasma wave one needs to disturb the plasma (plasma is assumed electrically neutral) by creating regions of excess negative and positive charge density. The unevenness of charge distribution generates an electric field which pulls together electrons and ions, but since electrons are much lighter than ions they are the ones which move towards the positive region. As they move they gain momentum and when they reach the positive region they overshoot and then will be pulled back creating a plasma 
wave oscillation. In order to accelerate, relativistic plasma waves (i.e. waves with phase velocity close to the speed of light) should be created and with the methods mentioned above it can be done.

The wakefields in a laser driven accelerator are generated via the ponderomotive force. As an intense pulse propagates through tenuous plasma $\left(\omega_{1}>>\omega_{p}\right.$, where $\omega_{1}$ is the laser frequency) the ponderomotive force associated with the laser envelope, $F_{p} \simeq-m_{e} c^{2} \nabla\left(a^{2} / 2\right)$ where $\vec{a}=e \vec{A} / m_{e} c^{2}$ is the normalized vector potential, expels electrons from the region of the laser pulse and excites electron plasma waves with a phase velocity equal to the laser group velocity. These waves are generated as a result of being displaced by the leading edge of the laser pulse. If the laser pulse length $\left(c \tau_{L}\right)$ is approximately equal to or shorter than the plasma wavelength, $c \tau_{L} \sim \lambda_{p}=2 \pi c / \omega_{p}$ the ponderomotive force strongly excites plasma waves or wakefields. [16]

Assuming the laser pulse maintains its shape, the final energy attainable in a plasma accelerator would be limited by several factors:

First and the most severe limit arises from energy of the laser pulse not being efficiently used in creating the plasma wave, this limit arises from light diffraction. The diffraction length of a laser pulse is $\pi w^{2} / \lambda_{L}$, where $w$ is the spot size of the laser beam and $\lambda_{L}$ is the laser wavelength. The generation of a high gradient requires the excitation of a large-amplitude plasma wave, which in turn demands an intense laser field and therefore a small laser spot size. This smaller spot size causes greater diffraction.

Second, the difference between the plasma wave phase velocity and the particle velocity $c$ leads to a detuning of the phase of the accelerating field [30,31]. As the 
electron is accelerated, its velocity $v_{z}$, will increase and approach the speed of light. If the phase velocity of the plasma wave is constant with $v_{p}<c$, the electrons will eventually outrun the plasma wave and move into region of the plasma wave which is decelerating. This limits the energy gain of the electron in the plasma wave and is referred to as electron phase detuning. The detuning length $L_{d}$ is defined as the length the electron must travel before it phase slips by one-half of a period with respect to the plasma wave. For relativistic electron $v_{e} \approx c$, the detuning time [3] $t_{d}=\pi v_{p} /\left[\omega_{p}\left(c-v_{p}\right)\right]$ and $L_{d}=c t_{d} \simeq \gamma_{p}^{2} \lambda_{p}$ when $\gamma_{p} \gg 1$, here $\gamma_{p}=\left(1-v_{p}^{2} / c^{2}\right)^{-1 / 2}$ and $v_{p}$ is phase velocity.

The third is pump depletion. As the laser pulse propagates in the plasma and excites wakefields its energy is coupled into plasma and the laser pulse eventually becomes depleted which in turn limits the amount of energy gained by the accelerated particles.

Two solutions have been proposed for overcoming diffraction: self focusing [32, 33, 55-59] and plasma channel guiding [34],. Both work by creating a higher index of refraction along the axis of the laser pulse than at the edges, thus slowing the wave phase front on axis relative to those at the edge and focusing and guiding the pulse. In the case of self focusing two effects: relativistic mass increase and ponderomotive expulsion of electrons are the main causes of the effect. The relativistic mass increase in the region of high power intensity lowers the effective plasma frequency and raises the dielectric constant $\left(\varepsilon=\left(1-\omega_{p}^{2} / \gamma \omega^{2}\right)\right)$. The ponderomotive force pushes the electrons towards the regions of lower field and causes a decrease of charge density at the center of laser pulse. This further lowers the plasma frequency and raises the dielectric constant. If the laser 
pulse has sufficient intensity, the propagation will be similar to that in an optical fiber and the self focusing and diffraction can counter balance each other; however the head of pulse is unfocused because the modification in the index of refraction happens on the plasma frequency time scale, not the laser frequency time scale. Relativistic self channeling can overcome diffraction if the pulse power is greater than critical power for relativistic self focusing $P>P_{c} \quad$ where $P_{c}[G W]=16.2 \omega_{l}^{2} / \omega_{p}^{2}[47]$. There are other methods to prolong the interaction of the laser with the plasma medium; such as capillary discharge[48], gas filled capillaries[49] and Plasma channels can be used to overcome diffraction if $P<P_{c}$.

Plasma channel guiding which was first pioneered by Howard Milchberg and his group from the University of Maryland is a possible alternative approach $[50,65]$. In plasma-channel guiding, the plasma's transverse density profile is modified so that its minimum is at the center of the laser pulse, giving rise to the higher index of refraction on axis. This guiding works independently of the laser power.

\subsection{Purpose of Dissertation}

In the present thesis the problem of self trapping of charged particles by the electromagnetic wakefield following an intense laser pulse (or electron beam) propagating through a low density plasma, is studied. This process is important to current and future particle accelerators that utilize plasma based acceleration processes; in particular Laser Wakefield Acceleration (LWFA) and Plasma Wakefield Acceleration (PWFA). The main focus of my work will be to modify the simulation code WAKE to 
describe this process and then to undertake an extensive numerical study of particle trapping.

P. Mora and T. Antonsen [2] introduced a 2D cylindrically symmetric code called WAKE based on the quasistatic approximation which would model the propagation of intense and very short laser pulse in a tenuous plasma. The advantage of their model is appreciated when one compares the same sets of parameters being simulated by both a full-PIC code and WAKE and the results would be remarkably accurate with orders of magnitude less amount of CPU hours spent.

In a full PIC code since the algorithm makes very few physics approximations and smallest spatial scales should be resolved, it is very intensive for amount of computing needed. As the number of stages of acceleration or the amount of energy increases it becomes apparent that using full PIC simulations become less desirable.

On the other hand because of the nature of plasma accelerators there are some approximations available to physicists to explore. In these accelerators the driver and the accelerated beam that follows evolve on two very different time scales in comparison to the plasma wake. In the PIC code the wavelength of laser needs to be resolved. On the other hand WAKE uses this difference in time scales with the quasistatic approximation to reduce the number of time steps significantly. The goal of this thesis is to accommodate WAKE with the capability of simulating acceleration and self consistently trapping process of the electrons in the wakefield [4].

We modify the code WAKE to allow it to simulate LWFA and also the capability to simulate the case of externally injected particles in the laser wakefield by defining two sets of particles. First are the background plasma particles, for which the quasistatic 
approximation is valid. Second, are the beam particles, which are assumed to be moving in the laser frame with velocity near speed of light. For these particles the QSA is not valid and their dynamic must be calculated more accurately.

The first step in successful modification of the code is being able to benchmark our results with some other reliable simulations or to find good agreement between the simulation results and the experimental data. To verify that the method of two separate sets of particles as beam and plasma is adequate and to study some other effects, the code was adjusted to the case, when the simulated laser pulse was off and wake was created by an externally injected, ultra relativistic, electron beam. These wakefields have been benchmarked with the OSIRIS simulation which is a fully explicit model. To study beam loading effects and to verify that aspect of the code the laser was turned back on and particles were injected in the wakefield created by the laser and by constructing the selfconsistency in the model the outcome was in accordance with the predictions of beam loading effects.

The next step is to create a scheme in which one can trap plasma particles from the background plasma and completely simulate LWFA. In this case instead of injecting beam electrons into the wake, plasma particles with enough energy would be promoted to beam status and treated as beam particles. Their charge and subsequent current density are calculated and added to the current density of wakefield. In doing so, we need to define a threshold for the energy of plasma particles as our trapping condition. Also it will be proved that there needs to be additional conditions in promoting plasma particles to beam status since in practice we bring $\gamma_{t}(\gamma$ threshold $)$ as low as possible. Removing all the promoted particles, using the scheme of choosing lowest energy threshold, to 
beam status from the background plasma causes 2 major problems: First, the code would slow down. Second and more severe one is the fact that solving all equations of motion and wakefields for the promoted particles creates some numerical noise and makes the results unusable.

The method of combining the wave breaking of the particles with threshold trapping is proposed to solve the mentioned problem. In this method in addition to using the minimum threshold of energy for promoting the plasma particle to beam status, if it also crosses the radial axis of simulation, only then it would be promoted to the beam status. Once a particle is promoted to beam particle status it is followed in the laser frame.

Because of our promotion algorithm we will be promoting particles to beam status which would not get trapped in the wake, at the same time these particles contribution to the background plasma calculations are no longer in effect which causes error in calculating the transverse current density. Therefore, although we do not need to calculate $j_{\perp}$ for beam particles, we do it so that the error for the promoted particles which later on do not get trapped is accounted for.

Finally the combined promotion method is tested with experimental results of LNBL [3] observation of GeV particles and also with OSIRIS full-PIC code in the case for the 200TW laser and in the "Bubble Regime" [4, 63]. These results show that code WAKE is now capable of simulating LWFA in orders of magnitude faster CPU time than that of fully explicit models such as OSIRIS [72], with remarkable agreements between the results and basically on a single CPU desktop computer. 


\section{$\underline{1.3 \text { Some definitions }}$}

- Plasma Particle: A particle with low energy for which the QSA is valid.

- Beam Particle: Validity of QSA fails for this particle and the equations of motion are solved completely for this particle.

- Promoted to beam particle status: If a plasma particle energy satisfies the trapping conditions, that particle is promoted to beam particle status (This status does not guarantee that the particle eventually becomes trapped).

- Trapped particle: A beam particle which becomes trapped in the wakefield and gets accelerated to high energy is called trapped particle. (this is a physical effect)

- Wave breaking: Normally when two particles trajectories cross this is called wave breaking, here we modify this definition and use it in the case where a particle trajectory crosses the radial axis of the cylindrically symmetric coordinate system in the simulation.

- Bubble regime: The term is used when and ultra-relativistic laser pulse with duration shorter than $\lambda_{p}$ is applied to the plasma and breaks the plasma wave after the first oscillation. The properties of this regime is as follows [47]:

i) A free from electron (bubble) cavity is formed behind the laser pulse.

ii) A dense group of nearly monoenergetic electrons is self generated.

iii) The laser pulse propagates for many Rayleigh lengths whitout significant diffraction, since by total electron cavitation in the region of laser pulse the charge density is constant so there would be no further growth of refractive index and a 
quasi-stationary structure can form, which means self channeling of the laser beam.

\subsection{Structure of the thesis}

The rest of the thesis is organized as follows. Chapter 2 presents the origins and the theory behind the implementation of the code WAKE and the modifications that are made to it; specifically to make it suitable for simulations of LWFA and externally injected particles in the wakefields created by laser pulse. Chapter 3, the trapping process using all the trapping conditions and for the case of "Bubble regime" are investigated and the code is benchmarked against OSIRIS. Chapter 4 discusses the results of comparing the experimental results form LBNL, $1 \mathrm{GeV}$ to the output of the modified code in the trapping process. Finally, in chapter 5, summary and conclusion in briefly presented. 


\section{Chapter 2: Code WAKE}

\subsection{History of WAKE}

WAKE [2] was developed to model the propagation of ultra short, ultra intense laser pulse in underdense plasma. Here underdense means that the electron density is much smaller than critical density $\left(n_{0} \ll n_{c}\right.$ where $n_{c}=\left(\omega_{l}^{2} m_{e} /\left(4 \pi q^{2}\right)\right)$ which is replacing $\omega_{l}$ instead of $\omega_{p}$ in calculating $\left.n_{0}\right)$.

WAKE is a fully relativistic, nonlinear, kinetic particle-in-cell (PIC) simulation tool, but it utilizes the disparity of time scales in the short pulse laser-under dense plasma interaction to significantly reduce computational times. The disparity in time scales allows for the application of two simplifying approximations.

The first approximation involves enveloping the laser pulse, which requires that the pulse shape evolves slowly compared with the laser period. Generally this will happen in underdense plasma. Enveloping the laser field results in two efficiencies: First, the wave equations no longer need to resolve the laser period. Second, the plasma motion can be separated into a rapidly varying quiver motion and a slowly varying ponderomotive response. The quiver motion of the plasma electrons can be averaged over, and as a result only the plasma electron motion due to the ponderomotive force of the driver and the plasma wakefields is considered (the quiver motion is, however, accounted for energetically). We note that the envelope approximation precludes consideration of backscatter. Typically backscatter is not an issue for short pulses [32] and the laser radiation is mostly forward propagating. 
The second approximation is the quasi-static or frozen field approximation: the driver laser envelope and its resulting wakefield do not change shape in the time it takes for it to pass by a plasma particle (implicit in this approximation is that the under dense plasma is cold, $T_{e}<<m_{e} c^{2}$ where $T_{e}$ is the electron temperature). This approximation can be expressed by the inequality $\tau_{L} \ll \tau_{E}$, where $\tau_{L}$ is laser pulse (or electron beam) duration, $\tau_{E}$ is the laser pulse evolution time, for example if diffraction is the dominant effect, then $\tau_{E} \sim \pi w^{2} / c \lambda_{0}$, here $w$ is the laser spot size, $\lambda_{0}$ is vacuum laser wavelength, and $c$ the speed of light. The difference in these times scales allows separation of Maxwell's equations (describing evolution of the electromagnetic fields) from Hamilton's equations (describing evolution of the plasma electrons). Numerical calculation of the electromagnetic fields can be limited to time steps much larger than those required for the electron motion (which have already been greatly reduced by considering only the ponderomotive response), saving considerable processing time.

The implementation of this kinetic model is done using Particle in Cell methods with the above assumptions being used (one can call the code semi-PIC code). A full PIC code like OSIRIS which later in the thesis we benchmark our code simulation results to it, works in the following manner, first, instead of accounting for every particle, a statistical sample of particles called macro-particles is taken. A macro-particle is a special particle which carries the weight of a large number of particles when used to calculate the fields exerted on other particles. In response to a given field, the macro-particle moves as if it were a regular charged particle. Then, these particles are loaded onto a spatially gridded simulation domain and the charge and current densities at the grid points are calculated. At last the field equations are advanced using these charge and current 
densities via Maxwell's equations and using the updated fields the particles new position and velocities are calculated via the relativistic equation of motion: $d P / d t=q[E+(V \times B) / c]$. In the full PIC code one needs to resolve the shortest spatial scale which for laser driver is it's wavelength, but in WAKE because of differences in time scales and evolution of laser on Rayleigh time scale we can have much larger step sizes.

The kinetic method has its advantages over a hydrodynamic description of the plasma. It allows to correctly describe, energetic or fast electrons being generated, plasma wave breaking (wave breaking in the general meaning of it), creation of total electron cavity in the channel and other phenomenon which can not be expressed using hydrodynamic models.

In developing the new WAKE we have to consider, plasma electrons which become trapped in the wakefield and are accelerated to high energy cannot be treated within the framework of the quasistatic approximation. These electrons can spend significant durations in the wakefield. Both the laser pulse and wakefield can evolve on time scales which may be less than the time the electrons spend within the pulse. These electrons are the most relevant for accelerator design and modifications for treating such particles should be included. In order to treat electrons whose energy gain precludes a quasi-static treatment and to allow for simulations of plasma wakefield acceleration (PWFA), the particles are divided into two distinct groups:

1) Plasma particles that are passed quickly by the laser driver and the wake.

2) Beam particles that are traveling at or near the speed of light. 
The evolution of these particles takes place on two time scales. The equations describing the beam electrons will result from unapproximated Hamilton's equations while the quasistatic equations describing the plasma particles result from the assumption that fields evolve slowly in time.

\subsection{Laser model}

The laser pulse is represented in the code [2] with high frequency vector potential $\overrightarrow{A_{L}}$. The envelope approximation is established in the form of a plane wave traveling at the speed of light:

$$
\overline{A_{L}}=\hat{A}_{\perp}\left(z, x_{\perp}, t\right) \exp \left(-i k_{0} \xi\right)+c . c .
$$

where $\hat{A}_{\perp}$ is the envelope term and is varied slowly compared to laser period. $k_{0}=\omega_{l} / c$ is the wave number of the laser central frequency $\omega_{l}$ and $\xi=c t-z$ which measures the distance back from the head of the laser pulse. Here $\hat{A}_{\perp}$ depends on time and space and by substituting (2.1) in wave equation can be determined:

$$
\left[\frac{2}{c} \frac{\partial}{\partial t}\left(i k_{0}-\frac{\partial}{\partial \xi}\right)+\nabla_{\perp}^{2}\right] \hat{A}_{\perp}=k_{p}^{2} \frac{\bar{n}}{\bar{\gamma}} \hat{A}_{\perp}
$$

here $k_{p}=\omega_{p} / c$ and the bar over quantities means averaging over high frequency period.

The left hand side of the equation represents the wave operators, although we have dropped the term $\partial^{2} / \partial t^{2}$ which is of the order of $\varepsilon^{2}$ where $\varepsilon=\omega_{p} / \omega_{l}$. By doing this the backscattering radiation is eliminated. Also it should be noted the mixed derivates which is of the order of $\varepsilon$ are retained, which allows the absorption of radiation due to creation of plasma wake to be modeled. Also, by including this term it allows the code to model 
pure forward Raman scattering. The right hand side includes the plasma response, neglecting the generation of harmonics of the laser radiation, which is justifiable for underdense plasma. Averaging in the code is done by summing the contribution of simulation particles. The model is applicable to arbitrary polarization of the radiation, although in our numerical simulation we assume linear polarization.

\subsection{Governing equations of the code for plasma particles}

In this section we develop the equations of the wake and motion of the plasma particles. In the following equations we are going to assume plane symmetry just to make it simpler to follow, by analogy one can extend the formulation to axial symmetry [2].

We assume that fields depend on two coordinates, $x$ and $z$, and that the plasma motion is in the $x-z$ plane. In this case the components of the electromagnetic field that are present are $E_{x}, E_{z}$ and $B_{y}$. Maxwell equation can be written as follows in Gaussian units:

$$
\nabla \times \vec{B}=\frac{4 \pi}{c} \vec{j}+\frac{1}{c} \frac{\partial \vec{E}}{\partial t} .
$$

The relevant components of (2.3) are,

$$
-\frac{\partial}{\partial z} B_{y}=\frac{4 \pi}{c} j_{x}+\frac{1}{c} \frac{\partial E_{x}}{\partial t}
$$

and

$$
\frac{\partial}{\partial x} B_{y}=\frac{4 \pi}{c} j_{z}+\frac{1}{c} \frac{\partial E_{z}}{\partial t} .
$$

In the quasi static approximation wakefields, depend dominantly on the variable, $\xi=c t-z$. Using the variable $\xi$ equations (2.4) and (2.5) can be rewritten: 


$$
\frac{\partial}{\partial \xi}\left(B_{y}-E_{x}\right)=\frac{4 \pi}{c} j_{x},
$$

and

$$
\frac{\partial}{\partial x} B_{y}-\frac{\partial}{\partial \xi} E_{z}=\frac{4 \pi}{c} j_{z} .
$$

We now introduce potentials $\phi$ and $\vec{A}$ to represent the fields. The single component $B_{y}$ can be generated by a vector potential, $\vec{A}=A e_{z}$. The field equations become:

$$
\begin{aligned}
& E_{x}=-\frac{\partial \phi}{\partial x} \\
& E_{z}=-\frac{\partial \phi}{\partial z}-\frac{1}{c} \frac{\partial A}{\partial t}=\frac{\partial \phi}{\partial \xi}-\frac{\partial A}{\partial \xi}=\frac{\partial}{\partial \xi}(\phi-A),
\end{aligned}
$$

and

$$
B_{y}=-\frac{\partial A}{\partial x}
$$

Substituting these in equations (2.6) and (2.7), we get the potential equations:

$$
\frac{\partial^{2}}{\partial \xi \partial x}(\phi-A)=\frac{4 \pi}{c} j_{x},
$$

and

$$
-\frac{\partial^{2}}{\partial x^{2}} A-\frac{\partial^{2}}{\partial \xi^{2}}(\phi-A)=\frac{4 \pi}{c} j_{z}
$$

Let's now define some dimensionless quantities to simplify the numerical implementation in the code and an auxiliary variable [71]:

$$
\begin{array}{ll}
G=\frac{\partial^{2}}{\partial \xi^{2}}(\phi-A), & \\
a=\frac{q \vec{A}}{m c^{2}}, & \varphi=\frac{q \phi}{m c^{2}},
\end{array}
$$




$$
\begin{array}{ll}
g=\frac{G}{4 \pi n_{0} e}, & \tilde{e}=\frac{q \vec{E}}{m \omega_{p} c}, \\
\tilde{j}=\frac{\vec{j}}{n_{0} e c}, & b_{y}=\frac{q B_{y}}{m \omega_{p} c}, \\
\tilde{\xi}=k_{p} \xi, & \tilde{x}=k_{p} x .
\end{array}
$$

And one more auxiliary variable:

$$
\psi=\varphi-a
$$

So now we can rewrite the equation in dimensionless form:

$$
\begin{aligned}
& b_{y}=-\frac{\partial a}{\partial \tilde{x}}, \\
& \frac{\partial^{2}}{\partial \tilde{\xi}^{2}} \psi=g, \\
& \frac{\partial}{\partial \tilde{x}} b_{y}=\left(g+\tilde{j}_{z}\right), \\
& e_{z}=\frac{\partial}{\partial \tilde{\xi}} \psi,
\end{aligned}
$$

and

$$
e_{x}=-\frac{\partial}{\partial \tilde{x}} \varphi=-\frac{\partial}{\partial \tilde{x}} \psi+b_{y}
$$

These are the field equations which are implemented in the code WAKE.

The equations of motion for the laser period averaged, momentum and position of a plasma particle are written [2]:

$$
\frac{d \vec{p}}{d t}=q\left(\vec{E}+\frac{\vec{V} \times \vec{B}}{c}\right)-\frac{q^{2}}{2 \bar{\gamma} m c^{2}} \nabla\left|\hat{A}_{\perp}\right|^{2}
$$

and $d \vec{x} / d t=\vec{V}=\vec{P} /(\bar{\gamma} m)$ is the average velocity and 
$\bar{\gamma}=\left(1+\left(\frac{\vec{p}}{m c}\right)^{2}+\left|\frac{q \hat{A}_{\perp}}{m c^{2}}\right|^{2}\right)^{1 / 2}$,

is the averaged relativistic factor. The last term in Eq. (2.20) represents the ponderomotive force of the laser, and the last term in Eq. (2.21) represents the quiver energy of the election in the laser field.

In the quasistatic approximation the Hamiltonian depends on $z$ and $t$ only through the combination of $\xi=c t-z$. So from the Hamiltonian equations we have:

$$
\frac{d P_{z}}{d t}=-\frac{\partial \bar{H}}{\partial z}=\frac{\partial \bar{H}}{\partial \xi},
$$

and

$$
\frac{d \bar{H}}{d t}=\frac{\partial \bar{H}}{\partial t}=c \frac{\partial \bar{H}}{\partial \xi}
$$

where $\bar{H}\left(P_{z}, \vec{P}_{\perp}, \vec{X}_{\perp}, \xi\right)=\bar{\gamma} m c^{2}+q \phi$ and $P_{z}=p_{z}+q A_{z} / c$ is the canonical momentum in the $\xi$ direction.

Equations (2.22) and (2.23) imply $\bar{H}-c P_{z}$ is a constant of motion. Using the fact that before the pulse and wake reach a particle we can determine the value of the constant, and we find:

$$
\bar{\gamma} m c^{2}+q \phi-c p_{z}-q A=m c^{2} .
$$

Again defining dimensionless quantities:

$$
\tilde{p}=\frac{\vec{p}}{m c}, \quad \beta=\frac{\vec{V}}{c}=\frac{\tilde{p}}{\bar{\gamma}}, \quad \tau=\omega_{p} t .
$$

Now we can rewrite equations (2.20), (2.21) and (2.24):

$$
\bar{\gamma}=\left(1+\tilde{p}^{2}+\bar{a}^{2}\right)^{1 / 2}
$$




$$
\frac{d \tilde{p}}{d \tau}=-(\tilde{e}+\beta \times \tilde{b})-\frac{1}{2 \bar{\gamma}} \nabla \bar{a}^{2}
$$

and

$$
\bar{\gamma}-\tilde{p}_{z}-\psi=1
$$

Thefore:

$$
\frac{d \tilde{x}}{d \tilde{\xi}}=\frac{d x}{d \xi}=\frac{\tilde{p}_{x}}{1+\psi},
$$

and finally we can calculate $x$ component of (2.26) using (2.19):

$$
\frac{d \tilde{p}_{x}}{d \tilde{\xi}}=\frac{1}{1+\psi}\left(\bar{\gamma} \frac{\partial \psi}{\partial \tilde{x}}-\frac{1}{2} \frac{\partial \bar{a}^{2}}{\partial \tilde{x}}\right)-b_{y} .
$$

We also have:

$$
\frac{d x}{d t}=v_{x}=c \frac{\tilde{p}_{x}}{\bar{\gamma}},
$$

and

$$
\frac{d \xi}{d t}=c-\frac{d z}{d t}=c-v_{z}=c\left(1-\frac{\tilde{p}_{z}}{\bar{\gamma}}\right)=c \frac{1+\psi}{\bar{\gamma}}
$$

If we solve (2.25) and (2.27) with respect to $\bar{\gamma}$ and $p_{z}$ :

$$
\tilde{p}_{z}=\frac{1+\tilde{p}_{x}^{2}+\bar{a}^{2}-(1+\psi)^{2}}{2(1+\psi)},
$$

and

$$
\bar{\gamma}=\frac{1+\tilde{p}_{x}^{2}+\bar{a}^{2}+(1+\psi)^{2}}{2(1+\psi)} .
$$

We can determine electron motion using (2.29), (2.32) and (2.33). 
As the laser pulse passes over an electron it contributes to the local density according to the amount of time it spends in a particular axial region. To calculate the density of plasma particles the following equation is used:

$n\left(x_{\perp}, \xi\right)=\frac{c}{c-v_{z}} n\left(x_{\perp 0}, 0\right)$

where $x_{\perp 0}$ is the transverse position before arrival of the laser pulse and $n\left(x_{\perp 0}, 0\right)$ is the upstream plasma density from the head of laser pulse.

All these equations are solved using finite difference scheme, where we have set up a set of grids in the radial and axial coordinates $\left(x_{\perp}, \xi\right)$. Some quantities are defined on the grid and some are defined on the half grid, as illustrated in the figure (2.1). The same illustration is done in figure (2.2) for the advancement of particles in the code.

The boundary condition is as follows: We assume the wake potential is regular at the origin, $\left(x_{\perp}=0\right)$ and vanishes at the boundary of simulation volume in radial direction. The particles whose trajectory crosses the radial axis are reflected from the axis. The laser field is treated with outgoing radiation boundary conditions on the radial boundary [2].

Every time step a new set of plasma particles enters the simulation, which means there is another tool to carry their information. As the plasma particles equations of motion (Eqs.2.29-2.33) are advanced the wakefields in that time step are calculated and the eventual laser field in response to the wakefields. The behavior of the plasma particles and their fields is reflected on the laser field of the simulation (Eq.(2.2)) and the evolution of the laser is carried from each time step to the next. 
It is possible now with the implementation of the above equations in the code, to simulate the laser plasma interaction. Figures (2.3), (2.4) and (2.5) are benchmarking of this part of the code in comparison to OSIRIS, a full PIC code with no QSA. These figures are plotted using the following parameters: $a_{0}$, which is defined as amplitude of normalized vector potential $a=q \vec{A} / m c^{2}$ of the laser in the mentioned normalized units, is $a_{0}=0.25$ for figure (2.3) and $a_{0}=1.0$ for figure (2.4) and $a_{0}=2.0$ for figure (2.5) which the last one represents $36 \mathrm{TW}$ laser. The rest of parameters are all the same for all figures. The sport size $r_{s}=8.2(\mu \mathrm{m}), \lambda=800(\mathrm{~nm})$ and uniform plasma density of $n_{0}=1.38 \times 10^{19}$. The longitudinal electric field $e_{z}$ (normalized to $m \omega_{0} c / e$ ) versus distance $\xi$ (normalized to $\left.c / \omega_{0}\right)$ is plotted as the laser travels from left to right after very short distance of propagation. Normalization is done to $\omega_{l}=\omega_{0}$ since for OSIRIS simulation the laser period should be resolved. Also note that in order to compare WAKE with OSIRIS we have to make, $a_{0}$ in the OSIRIS simulation to be double the one used in WAKE, because of the way equations are implanted in the two codes.

We can see very good agreement between WAKE and OSIRIS for the moderate laser powers, corresponding to $a_{0}=0.25$ and $a_{0}=1.0$ but for more powerful lasers, corresponding to $a_{0}=2.0$ in figure (2.5), WAKE shows some difference with OSIRIS results. This is due to the creation of energetic particles and will be investigated subsequently. 


\subsection{Equations of the code with beam particles added to the system}

We start by considering the equation of motion for an electron in the combined laser and wakefield:

$$
\frac{d \tilde{p}}{d t}=c \nabla \phi_{W}+\left[\frac{\partial}{\partial t}-\left(\frac{c \tilde{p}}{\bar{\gamma}} \times \nabla\right) \times\right]\left(\vec{A}_{W}+\vec{A}_{L}\right)
$$

where the subscript $W$ refers to wakefield, we have used $\nabla \cdot \vec{A}_{L}=0$ in a tenuous plasma, and chosen a gauge for which $\phi_{L}=0$. In addition, the momenta, scalar potential, and vector potential have been normalized by $m_{e} c$ and the fundamental unit of charge $e$ is included in the potentials. Introducing the laser coordinates $\xi=c t-z$ and $s=c t$. The time derivative in equation (2.35) is written:

$$
\frac{d}{d t}=\left[\frac{\partial}{\partial s}+\left(1-\frac{\mathrm{v}_{\mathrm{z}}}{\mathrm{c}}\right) \frac{\partial}{\partial \xi}+\frac{\overrightarrow{\mathrm{v}}_{\perp}}{c} \cdot \nabla_{\perp}\right]
$$

Where $\partial / \partial s$ and $\partial / \partial \xi$ respectively are, slow and fast time derivatives. Although both $\xi$ and $s$ have units of length they represent fast and slow time scales. The two time scales in WAKE are interpreted as follows: the fast time scale (short numerical time step) is used for plasma evolution with respect to the coordinate $\xi=c t-z$. In the stationary frame of the driver, the plasma electrons stream past at near luminal velocities. Their evolution in response to the driver occurs much more rapidly than the driver response. Because of the large electrostatic force created by the expulsion of electrons from the path of the driver, some of the plasma electrons can reach relativistic energies and may be trapped by the resulting wakefield. As with the driver, these electrons evolve on the slow time scale (long numerical time step) with respect to coordinate $s$. 
Eq. (2.35) becomes:

$$
\begin{aligned}
& c \frac{d \tilde{p}_{\perp}}{d t}=\nabla_{\perp} \psi+\left(1-\frac{\mathrm{v}_{\mathrm{z}}}{\mathrm{c}}\right)\left[\nabla_{\perp}\left(A_{W, z}+A_{L, z}\right)+\frac{\partial}{\partial \xi}\left(\vec{A}_{W}+\vec{A}_{L}\right)_{\perp}\right] \\
& \frac{d p_{z}}{d s}=-\frac{\partial \psi}{\partial \xi}-\frac{\overrightarrow{\mathrm{v}}_{\perp}}{c} \times\left[\nabla \times\left(\vec{A}_{W}+\vec{A}_{L}\right)\right]
\end{aligned}
$$

where $\psi=\phi-z \cdot\left(\vec{A}_{W}+\vec{A}_{L}\right)$ and we have taken $\partial(\phi, \vec{A}) / \partial_{s}=0$. Taking $\partial(\phi, \vec{A}) / \partial_{s}=0$ is consistent with the envelope approximation: the wake and driver potentials are essentially steady state over a background electron transit time.

For beam particles, we solve the full version of Eqs. (2.37) and (2.38). Previous works [17] have treated the beam particles in the limit of $\mathrm{v}_{z} \sim c>>\left|\overrightarrow{\mathrm{v}}_{\perp}\right|$. In this limit, Eqs (2.37) and (2.38) reduce to

$$
\begin{aligned}
& \frac{d \vec{p}_{\perp}}{d s}=\nabla_{\perp} \psi \\
& \frac{d p_{z}}{d s}=-\frac{\partial}{\partial \xi} \psi
\end{aligned}
$$

This approximation requires a lower bound on the energy for which a particle can be considered a beam particle $\left(1-\mathrm{v}_{\mathrm{z}} / c\right.$ must be small). Such a treatment is not suitable for handling the transition of electrons from a background to a trapped state. By solving Eqs. (2.37) and (2.38) fully, no such approximation is required.

\subsection{Algorithm of Self-consistent beam dynamics}

In order to make the code self consistent we need to add the current density contribution from the particles with beam status to the current density of plasma particles. This should happen regardless of whether the particle is a self trapped one or injected 
beam particle. When a particle is promoted to beam status it contributes to the current density as a beam particle, and its contribution as a plasma particle is turned off. The current density calculated in the code as follows:

1) Particle positions are updated on the long time scale.

2) Particle positions fall between grid points for fields.

3) Field values are interpolated to particle positions to compute forces.

4) New particle positions are found.

5) Currents on grids are computed by assigning particle contributions to four nearest grid points as shown in figure 2.6.

In order to find the amount of charge and eventually the current density the following equations are implemented. Each beam particle represents an amount of charge $\Delta q_{i}$ that is distributed to its four nearest grid points in the $r-\xi$ plane with weighting factor $w_{i}(r, \xi)$ where $(r, \xi)$ represents a point on the grid. Similarly the contribution to current density $\Delta q \vec{v}$ are accumulated on each grid point. The beam charge density $\rho(r, \xi)$ is given by:

$$
\rho(r, \xi)=\sum_{i} w_{i} \frac{\Delta q_{i}}{\Delta r \Delta \xi}
$$

where $\Delta r$ and $\Delta \xi$ are the radial and axial grid sizes. The current density is given by:

$$
\vec{j}^{\text {beam }}=\sum_{i} w_{i} \vec{v} \frac{\Delta q_{i}}{\Delta r \Delta \xi}
$$

In cases in which externally injected beam particles are simulated the particle charges $\Delta q_{i}$ are adjusted to give a prescribed total beam charge $Q_{0}=\sum_{i} \Delta q_{i}$. In cases where plasma particles are promoted to beam particle status: 


$$
\Delta q_{i}=\frac{1}{N_{p}} 2 \pi r_{0} \Delta r n\left(r_{0}\right) e c \Delta t
$$

where $r_{0}$ is the initial launch radius of the plasma particle and $n\left(r_{0}\right)$ is the plasma density at that point and $\Delta t$ is the long time step.

In computing the current density we retain both the radial and axial components. High energy beam particles contribute much more strongly to the axial current density. However since we will simulate particles that have moderate energies we retain both component of current density.

In the next slow time step the contributions of promoted particles is added in accordance to conservation of charge in the system.

$$
\vec{j}=\vec{j}^{\text {plasma }}+\vec{j}^{\text {beam }}
$$

Where $\vec{j}^{\text {plasma }}$ is current density contributed from plasma particles and $\vec{j}^{\text {beam }}$ represents the contribution from beam particles.

\subsection{Externally injected particles simulation in WAKE and Benchmarking}

The addition of a population of beam particles to the WAKE model enhances the capability of the code to simulate plasma based particle acceleration. Since the self consistent effects of the charge and current density of the beam particles on the wakefields is now calculated, WAKE can be used to study beam loading in the case of LWFA and it can also be used to simulate PWFA. In this latter case we remove the laser pulse from the simulation. In this section we illustrate these capabilities and benchmark the results of the new WAKE algorithm against existing codes. 
The first case we consider is that of PWFA. For this case we turned the laser pulse off and initialized the beam particles to represent a relativistic beam with a Gaussian profile, with width of $\sigma_{\perp}=7 \mu \mathrm{m}$, length of $\sigma_{\mathrm{Z}}=45 \mu \mathrm{m}$ and charge $Q_{0}=2.88(n C)$ and the plasma density of $n_{0}=2.0 \times 10^{16}\left(\mathrm{~cm}^{-3}\right)$.

The results are displayed in figure (2.7(a)) where the longitudinal wakefield $e_{z}$ according to Eq. (2.19) on axis $(r=0)$ normalized to $m c \omega_{p} / e$ is plotted versus distance back from the charge bunch normalized to $c / \omega_{p}$. Also shown on the same plot is the longitudinal field produced by a QuickPIC [17] simulation with the same parameters. QuickPIC is a 3D code which treats the plasma in the QSA and has a separate population of beam particles similar to what we have just implemented in WAKE.

In these two graphs the driver e-beam moves slowly toward the left. Plasma electrons, enter the simulation domain from the left, are pushed laterally by the fields of the beam and are then pulled inward by the ion charge density that is exposed in this process. When the electrons are pulled inward they cross the axis at $\xi_{c} / \omega_{p}=6$ creating a large electron density spike. This gives rise to the sudden jump at $\xi_{c} / \omega_{p}=6$ in the wakefield. As can be seen WAKE and QuickPIC produce essentially the same longitudinal wakefield. Since both QuickPIC and WAKE make similar approximations to the plasma motion it is not surprising that they agree. Figure (2.7(b)) show a comparison of QuickPIC and OSIRIS (a full PIC code with no QSA) taken from [17]. Thus, we see that results from the two codes based on the QSA agree quite well with the full PIC 
results as well. Also the surface plot of $e_{z}$ and the plasma density profile of the system is plotted in figures (2.8) to better illustrate the conditions.

With the code capable of describing the contribution of the beam to the plasma wakefields we were also able to see the effects of beam loading on acceleration as is shown in figure (2.9). In this case we simulate a laser pulse with $\lambda=800(\mathrm{~nm}), a_{0}=1$, the spot size $r_{s}=25(\mu \mathrm{m})$. The electron beam is injected in the wakefields created by the laser where $\mathrm{Q}_{0}$ is the same as before. The histogram of the energy distribution of particle at the time of $t=0.8 t_{r}$ where $t_{r}=\omega_{l} r_{l}^{2} / 2 c^{2}$ is Rayleigh time, is calculated and depicted in figure (2.9). Three separate amounts of charge, each is bigger one order of magnitude than the other, is injected into the wakefields created by laser pulse. The spectrum shows, as the amount of injected beam charge increases the number of particles and the energy to which they are accelerated goes down.

\subsection{Trapping Process in the code}

For simulations of recent LWFA experiments the code needs to be able to treat the case of self-trapping. In the self-trapping regime, all electrons start as plasma particles. Some of these electrons will be accelerated by the wakefield to high energies. In order to treat trapping, a method must be implemented for "promoting" a plasma particle to a beam particle.

In this section we explore different algorithms for making this promotion. First we give some general consideration regarding the trapping process. Then we introduce two criteria for promotion, one based on particle energy and one based on a particle coming near the axis when fields are high (wave breaking). We then investigate these 
criteria by artificially considering the promoted particles to be passive, i.e. to not contribute to the plasma wake. In this way varying the criteria does not change the properties of the WAKE.

The primary distinction between plasma particles for which the QSA is valid, and beam particles for which the full equations of motion must be solved, is that plasma particles pass through the laser pulse and wake in a short time, during which the wake does not change. Whereas, beam particles either spend a long time in the wake, during which the wake changes, or they become trapped in the wake. The simplest criteria for distinguishing these two classes of particles is energy. Specifically high energy particles, which also mean high axial momentum particles since $p_{z} \gg p_{\perp}$ in most cases, should be considered as beam particles. Thus we first consider a threshold on energy for promotion.

As it stands the threshold energy is somewhat arbitrary. An estimate can be made, however, for the threshold value of $\gamma$ from simple physical considerations. The group velocity of laser is given by $v_{g}=c\left(1-\omega_{p}^{2} / \omega_{l}^{2}\right)^{1 / 2}$, and the velocity of the electron by $v_{z}=c\left(1-1 / \gamma^{2}\right)^{1 / 2}$. When these two velocities are equal the time an electron remains in the laser frame is indefinite. Equating the two velocities to determine $\gamma$ we find: $\gamma=\omega_{l} / \omega_{p}$. As an example consider a laser wavelength $\lambda=800 \mathrm{~nm}$ and a plasma density of $1 \times 10^{18} \mathrm{~cm}^{-3}$, which gives $\gamma \cong 42$, for relatively small values of $\gamma$ the particles start to outrun the laser. Therefore, if the quasi-static approximation is to be valid for plasma particles, $\gamma_{t}$, which is defined as the threshold value for gamma, should be much smaller than $\omega_{l} / \omega_{p}$. We will find however that this argument vastly overestimates the threshold. 
To make a better estimate of the lower limit on energy we do another simple calculation. We consider the Hamiltonian of the system in the 1D limit $H=\gamma m c^{2}+q \phi$ where $\phi=\phi_{0} \cos \left(k_{p}\left(z-v_{g} t\right)\right)$ and here $v_{g}$ is the group velocity of laser since the potential is traveling with the group velocity of the laser. Hamilton's equations lead to the relation $H-v_{g} p_{z}=$ const. (note that in comparison to Eq. (2.28) here we put group velocity instead of speed of light since we are interested in trapped particles). From this, we can plot contours of the constant in the $p_{z}$ versus $\theta=k_{p}\left(z-v_{g} t\right)$ plane as it is done in figure (2.10) and the blown up version of it figure (2.11). In both figures, $\phi_{0}=0.3$ and normalized group velocity is 0.95 . The plots of contours are drawn with changing constant from 0 to 1 . The closed contours represent trapped particles, while the open contours correspond to particles passed over by the wake. We can calculate the minimum value of $p_{z}$ on the separatirx, separating open and closed contours for different values of group velocity and potential.

The minimum value of $p_{z}$ on the separatrix for different potential amplitudes and group velocities is plotted in figure (2.12). When the potential amplitude is small, $q \phi_{0} / m c^{2} \rightarrow 0$, the estimate of the minimum momentum becomes $p_{z, \min }=\beta_{g}\left(1-\beta_{g}^{2}\right)^{-1 / 2}$, which is equivalent to our previous estimate. However, for potentials on the order of $0.15<q \phi_{0} / m c^{2}<0.30$ the minimum value for $p_{z}$ is less than 2 . Thus, it should not be surprising that we will find that the energy threshold can be very small. In the case of high power lasers and conditions of the "bubble" regime $\phi_{0}$ can become much bigger 
than the maximum 0.3 depicted in the plot, which lead us to believe very low values for threshold should be considered to distinguish beam and plasma particles.

The threshold can then be bounded by the inequality $1 \approx \gamma_{t}<<\omega_{l} / \omega_{p}$. In practice, we use values of $\gamma_{t} \sim 1$ to allow for an accurate handling of the transition of an electron from a background to a trapped state. The effects of choosing various threshold values are discussed in section 2.8. We note that the plasma particle description is encompassed by the beam particle description. Thus, by setting a low threshold we still describe all trajectories accurately, but at the cost of increasing computation time. If $\gamma_{t}=1$, the simulation would become a traditional PIC code and there would be no gain in computation speed (although still the laser period is not resolved). The low value of $\gamma_{t}$ implies that many particles will be promoted that don't become trapped. This is one of the reasons why the wave breaking condition is added to complement the threshold trapping process.

There are also some other disadvantages to having a low value for $\gamma_{t}$ as the only promoting condition of particles excluding the increase in the computation time or CPU hours needed for simulations. When the laser is not too powerful and the number of energetic particles are not more than a few percentage of the total number of plasma particles in the simulations the threshold trapping works adequately. But as the laser power increases and the system approaches the "bubble" regime this condition no longer is sufficient. The reason being, from the very beginning of the simulation energetic particles are being generated and they should be promoted and removed from background plasma. Removing the particles can introduce a certain amount of, numerical noise in the system, which gets magnified as the number of promoted particles increases. But as we 
discus in section 2.8 not all the particles promoted to beam status actually get trapped in the wakefield and accelerated to high energies most of these particles leave the simulation box without gaining much energy. The condition of introducing wave breaking is a tool to limit the number of unnecessary promotion of particles without significantly affecting the amount of charge being accelerated to high energies. So as a result not only the code would run much faster but the amount of noise decreases considerably.

\subsection{Laser Wakefield Acceleration in WAKE}

With the promoting and trapping algorithms in place, LWFA simulations with WAKE can be done. Plasma particles which satisfy the promotion conditions will be promoted to beam status and treated as beam particles, in the new WAKE. For all the graphs in this section we used the parameters of LBNL experiment [3]. Where, the interaction occurred in a capillary discharge, about $33 \mathrm{~mm}$ long with diameter of $310 \mu \mathrm{m}$, a Ti:sapphire laser $(\lambda=810 \mathrm{~nm})$ with $40 \mathrm{TW}$ peak power and sport size of about $25 \mu \mathrm{m}$ at the capillary entrance was used. For these parameters electrons up to $1 \mathrm{GeV}$ in energy were observed.

\subsubsection{Study of particle trajectories}

The best way to explore the differences between the plasma and beam particle algorithms is to investigate the trajectories of particles first when they are in plasma mode and then when they became trapped in the wakefield and are treated as beam particles.

Figure (2.13(a)) shows four trajectories in the $r-\xi$ plane. Each set of two (plasma and beam particle) shows the same particle that enters the simulation domain 
from the left. Here the laser pulse occupies the shaded region shown in the figure and is moving to the left so that in the frame of the laser pulse $(\xi=c t-z)$ plasma particles enter on the left. For the dashed curve labeled plasma1, the plasma electron is first pushed outward in radius by the ponderomotive force and then pulled inward by the uncovered plasma ion charge. The electron crosses the axis and is expelled from the simulation domain. The trajectory of the same particle, but simulated as a beam is also shown with a solid line. It follows the plasma electron trajectory for some time, but once its energy becomes high enough, it becomes trapped in the wakefield and starts its oscillation around the radial axis and is accelerated to an energy of about $750 \mathrm{MeV}$. The next set of two trajectories, beam 2 and plasma 2 shows the trajectory of a particle treated with the two different algorithms in a case where the particle does not become trapped and consequently both algorithms produce the same trajectories. Figure (2.13(b)) shows another example of particle trajectories for trapped particles. In this figure the beam particle trajectory oscillates around axis. The plasma particle trajectory it follows roughly the same path as figure (2.13(a)) only with more oscillations around axis.

One important result to be noted here, is the fact that, particles become trapped mostly when wave breaking occurs and in the figures (2.13(a) and (b)) it is clearly shown that when a plasma particle starts crossing the axis it becomes trapped in the wakefield. This and extensive study of trajectory of particles in both beam and plasma algorithms, justifies the fact that using the condition of wave breaking to promote plasma particles is a legitimate one.

In figure $(2.14$ (a)) the trajectory of a plasma and a beam particle is shown. Although in this case the particle did not stay trapped and left the simulation as a beam 
particle, it is depicted here to illustrate the exact position behind the laser pulse where the particle becomes trapped. This is shown in figure (2.14(b)). The particle became trapped when the wakefield became positive, and in the accelerating mode, which is what one would expect to happen.

\subsubsection{Study of the effects of selecting different thresholds}

In order to better understand the effects of different values of threshold on the amount of charge being accelerated to high energies, we did simulations for a range of threshold values, without having the condition of wave breaking implemented in the code.

The graphs in figures (2.15) and (2.16) demonstrate the amount of accelerated charge versus the energy threshold for promotion. In each figure the total remaining beam particle charge in the simulation (after $1.4 \mathrm{~cm}$ propagation in capillary) is depicted in red. And respectively the amount of charge with energy above $250 \mathrm{MeV}, 500 \mathrm{MeV}$ and 750 $\mathrm{MeV}$ are plotted in blue, green and black.

In figure (2.15) the effect of changing the threshold on the system where the beam particles are passive is shown. In other words the self consistency is removed meaning the charge of beam particles and therefore the current density is not added to $\vec{j}_{\text {plasma }}$ of the wakefields as discussed in Eqs. (2.41)-(2.44). It can be seen that as the threshold increases the number of promoted particles goes down till there will be no particles which satisfy the criteria for promotion. But the more significant fact is that as

one lowers the threshold it reaches a point where by further lowering it the number of particles accelerated to high energies does not change and reaches a plateau as illustrated 
in figure (2.15)). The fact that the plateau exists only for threshold energies below $\gamma=1.2$ indicates that the threshold must be set low to capture all particles that become trapped. On the other hand for the simulation with $\gamma_{t}=1.1$ the total amount of charge that was "promoted" was about $60(\mathrm{nC})$, whereas only $23(\mathrm{nC})$ remained in the simulation at the end and only $15(\mathrm{nC})$ reached $750 \mathrm{MeV}$. This shows that most promoted particles do not become trapped. Thus, we will seek a more refined criterion for promotion of particles.

Figure (2.16) shows the amount of trapped charge as a function of threshold energy computed when the beam current density contributes to wakefields. In this case the trapped charge is lower than in the case where beam particles are passive due to the "loading" of the wake by the beam particles. In addition the amount of trapped charge is less sensitive to the threshold in this case.

\subsubsection{Implementing Wave breaking condition}

We have defined the wave breaking condition to be when a particle starts to cross the radial axis of the system. With each iteration of the simulation the particles position is advanced. In order to promote the particles which would cross the axis, we check if the particle has reached a certain distance from the axis. One also has to be aware that the particles which intersect the symmetry axis are specularly reflected. As a result this distance should satisfy the fact that there may be a scenario in which, if it gets set too close to axis the particles in one time step are not yet in the range to be promoted and in the next step because of the reflection they may not be in the range again although they 
have crossed the axis. On the other limit, if the distance gets set too high then it loses its meaning.

In the figure (2.17), the amount charge of particles reaching energies above $750 \mathrm{MeV}$ with respect to variation in threshold distance from the axis to promote the particles is shown, after $1.4 \mathrm{~cm}$ propagation in the capillary. It clearly can be seen for some very small distances very important particles did not get promoted, on the other hand when the distance is set too high $(0.46$ and $0.57 \mu \mathrm{m}$ both produce the same amount of high energy particles) the result looks the same but much more number of particles are promoted and more CPU hours being wasted. In the case of $0.46 \mu \mathrm{m}$ the total promoted charge is $0.343(\mathrm{nC})$ but for $0.57 \mu m$ the conditions promoted 0.441 (nC) while producing the same amount of high energy particles. So the basic rule of thumb would be one has to consider the time between each time step and the speed particles may have before wave breaking to adjust for the calculation of the proper value of distance from the axis.

\subsubsection{Simultaneous implementation of promotion conditions}

The conditions of wave breaking and energy threshold are both implemented in this section. With the wave breaking condition fixed at $0.46 \mu m$ ( 0.2 in normalized units), we vary the energy threshold to compare the new results with the ones depicted in figures (2.15) and (2.16) for the same simulating conditions.

Now the code is running in the mode of LWFA simulation except in this chapter we do not yet remove the promoted plasma particles to beam status from the background plasma (meaning they still continue to be plasma particle also). We are only creating a 
small amount of error by not considering the removal of these particles from background since in LNBL experiment there are more than 0.2 (C) charge in the capillary and we promote less than tens of $(\mathrm{nC})$ charge in the whole simulation length. The omission of these particles is implemented and discussed in the next chapter when we compare WAKE with OSIRIS simulation.

First we consider the case of passive electron beams as was done in figure (2.15). The trend of the curves in figure (2.18) is the same as (2.15) in that both figures show flattening at low and less promotion at high energy thresholds. The differences are that we have gone from nearly $60(\mathrm{nC})$ promotion to $5.5(\mathrm{nC})$ and only nearly $2.3(\mathrm{nC})$ of them remains in the simulation. Also it should be noticed that in figure (2.18) the lines that represents different levels of energy are more tightly spaced which indicate less amount of energy spread.

When the self consistency is restored we have plotted figure (2.19) in the same terms as the plot in figure (2.16). Again we can see that both plots are following the same trend as the energy threshold decreases. In figure (2.19) the amount of total charge in the simulation at the time of $1.4 \mathrm{~cm}$ propagation in the capillary is cut by a factor of nearly 4 in comparison to plots of figure (2.16). On the other hand figure (2.20), which is the ratio of the charge above $750 \mathrm{MeV}$ for the cases in figures (2.16) and (2.19) shows for the range of $\gamma_{t}$ values of interest, above $80 \%$ of particles are captured using the new criteria. Although we cut the number of promoted particles by a factor of 4 , the number of accelerated particles was not affected much. Furthermore, if the number of promoted particles becomes large, the wakefields become noisy due to numerical effects. Thus, it is not even clear, that simulations with more promoted particles are more accurate. 


\subsection{Summary of the chapter}

In this chapter we started by introducing the origins of WAKE and its governing equations. It was then proposed in order to be able to simulate self-trapping process we need to consider two sets of particles, plasma and beam particles. The beam particles are not bound by quasi-static approximation and can become energetic. Then we introduced two criteria on how to promote a plasma particle to beam particle status. First by introducing an energy threshold for plasma particles and then by defining the wave breaking condition which follows the trajectory of plasma particles, as they are crossing the radial axis of simulation. The self consistency of beam dynamics is also discussed. As we promote plasma particles to the beam status we calculate their current density and add that to the current density of the system and also remove them from contributing to background plasma.

Then we have done extensive study of the effects of setting each promotion condition and how we can achieve the most physical conditions to be implanted. It was concluded that we need to set the energy threshold as low as possible to accommodate all the possible energetic particles, and on the other hand the wave breaking condition is implemented to limit the number of particles which would get promoted and do not become trapped in the process. 


\section{$\underline{2.10 \text { Figures }}$}

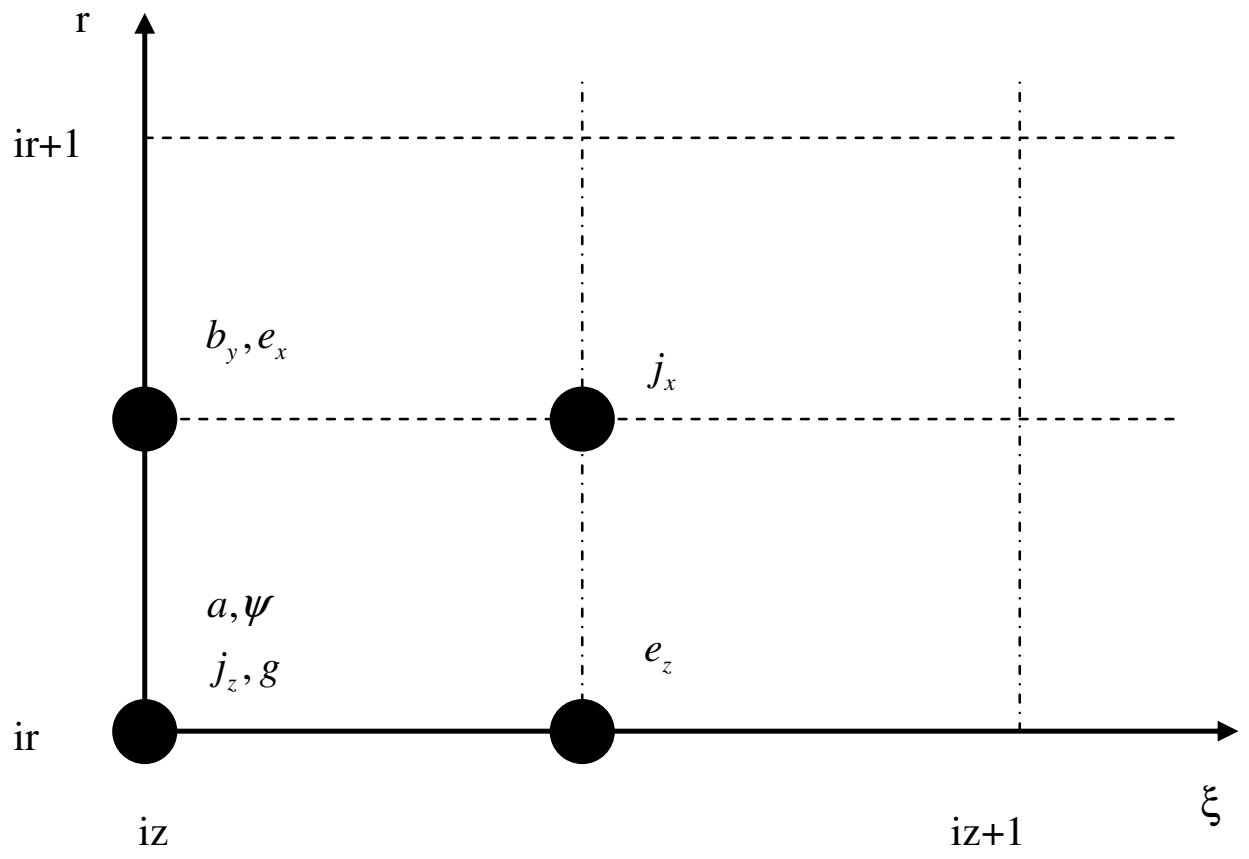

Fig.2.1) Diagram of how physical quantities are defined on the grid. 


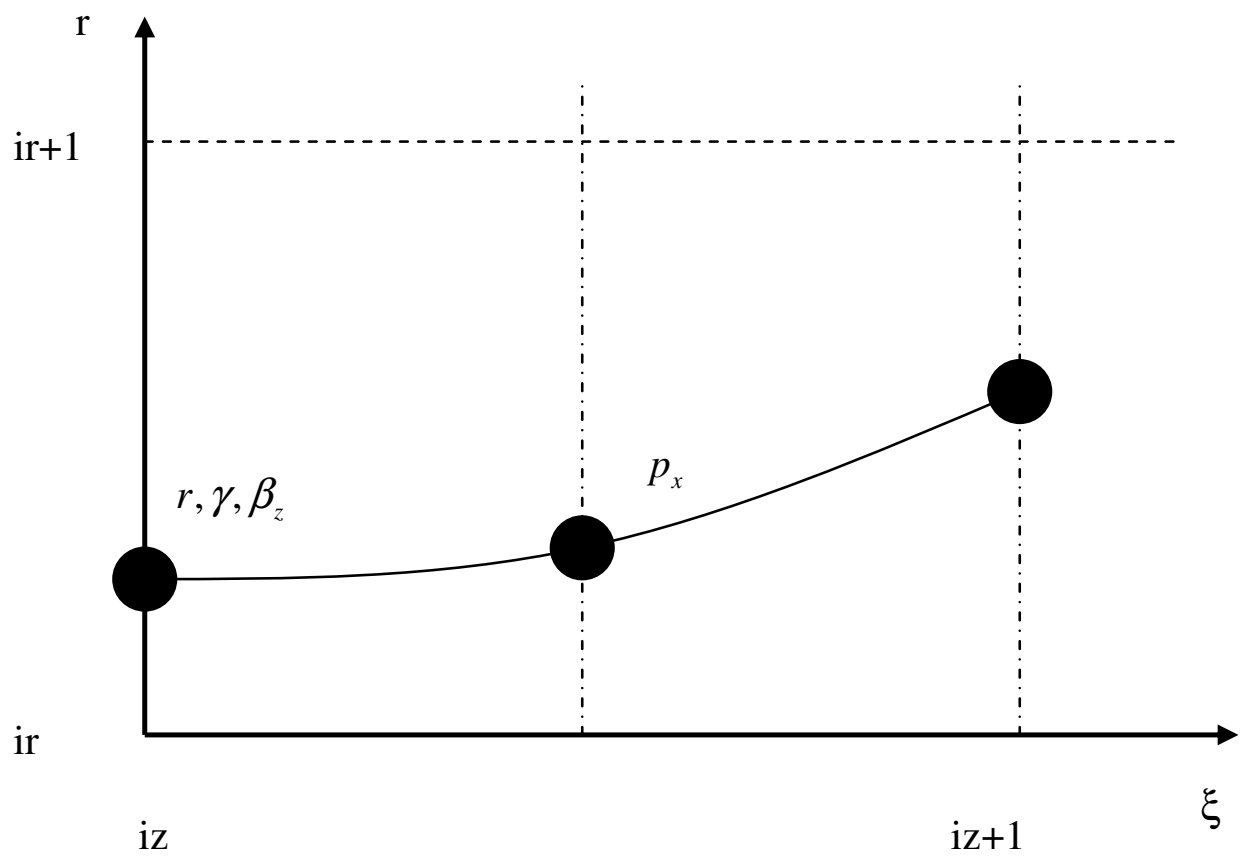

Fig.2.2) following the particles on the gird 


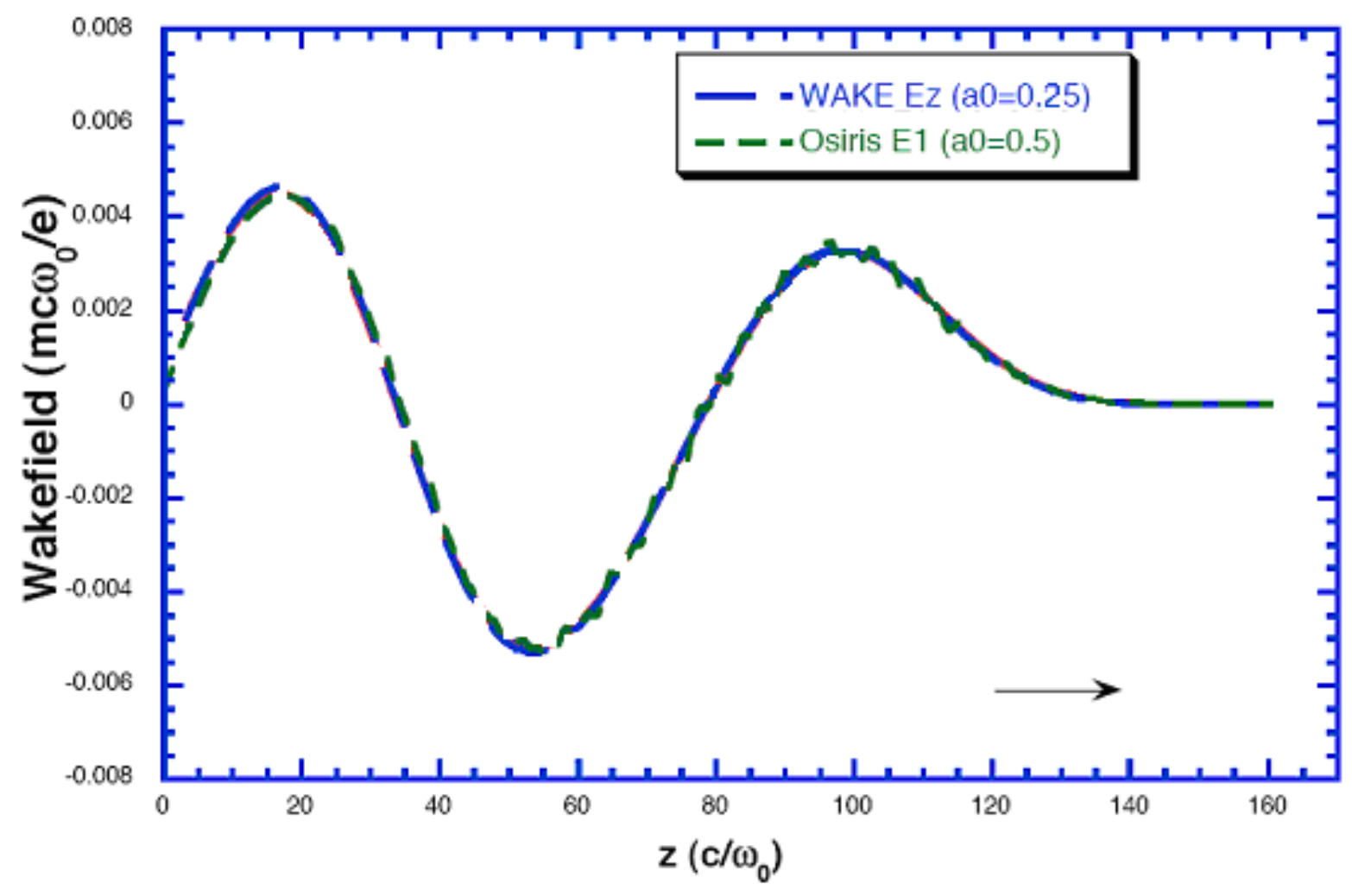

Fig.2.3) Comparison of WAKE with full PIC code, OSIRIS, without implementation of beam particles. The case of low power laser shows very agreement between them. 


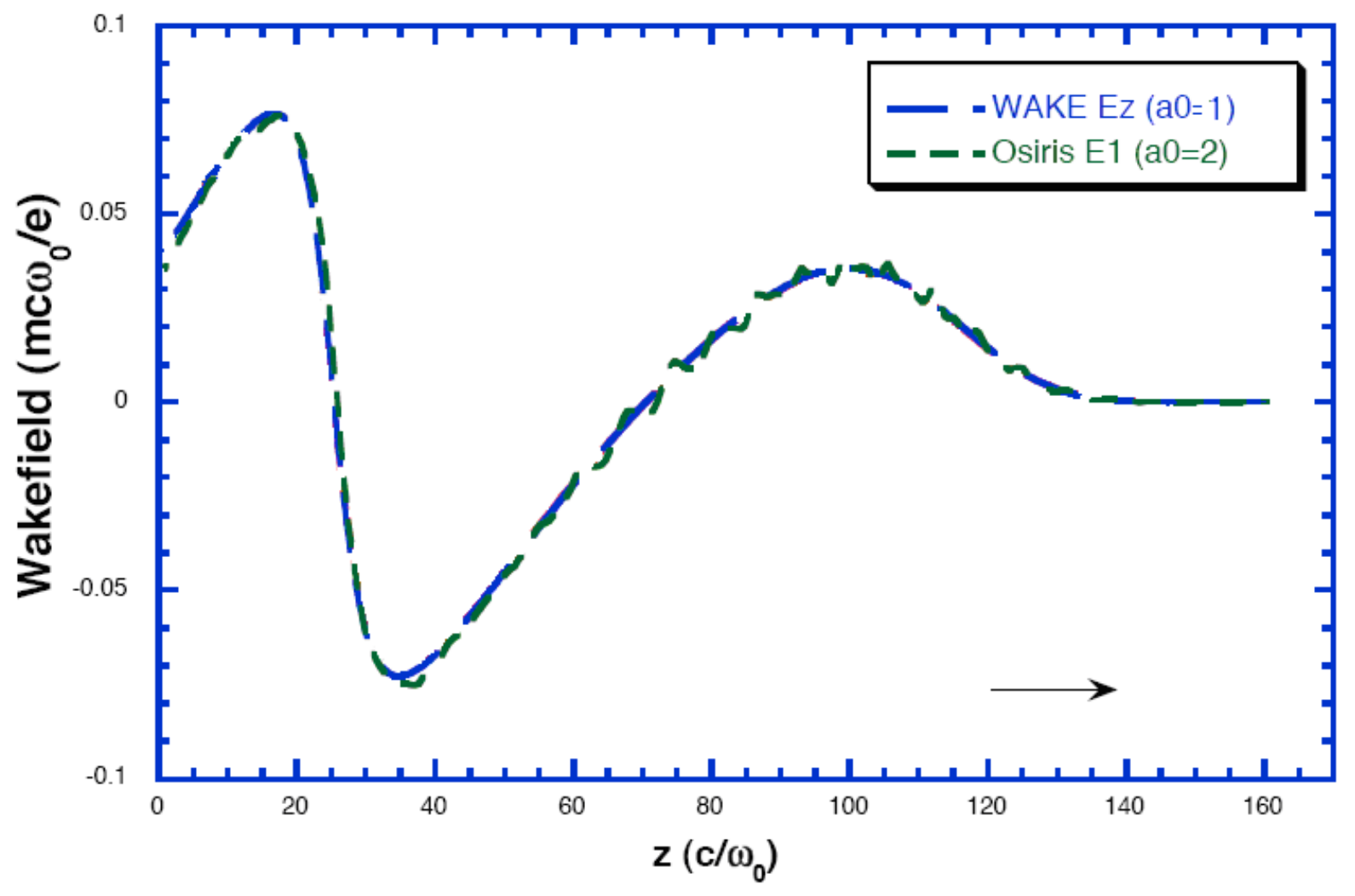

Fig.2.4) Comparison of WAKE with full PIC code, OSIRIS, without implementation of beam particles. The case of moderate power laser shows very agreement between them. 


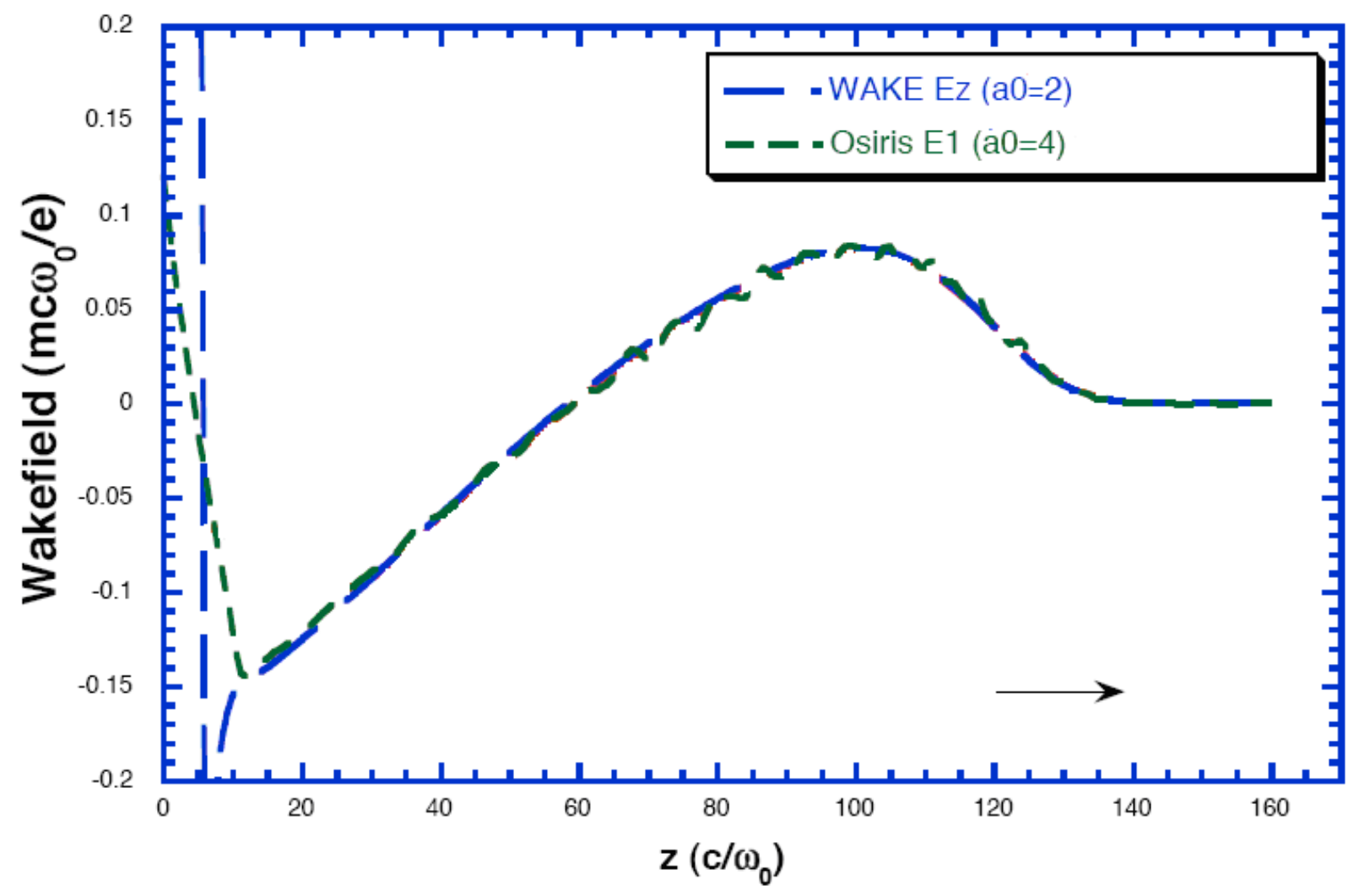

Fig.2.5) Comparison of WAKE with full PIC code, OSIRIS, without implementation of beam particles. The case of High power laser shows that WAKE can not handle these conditions and modifications are required. 

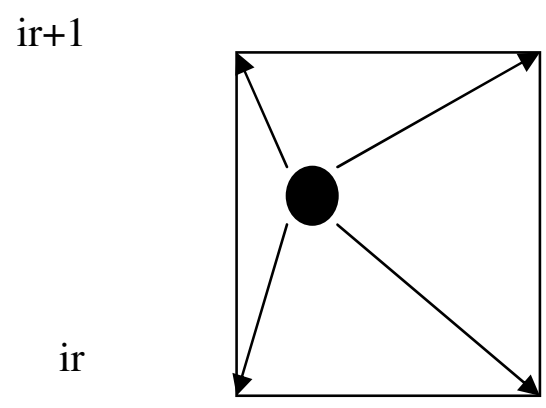

iz

iz+1

Fig.2.6) weighting of beam particles on the nearest grid points 

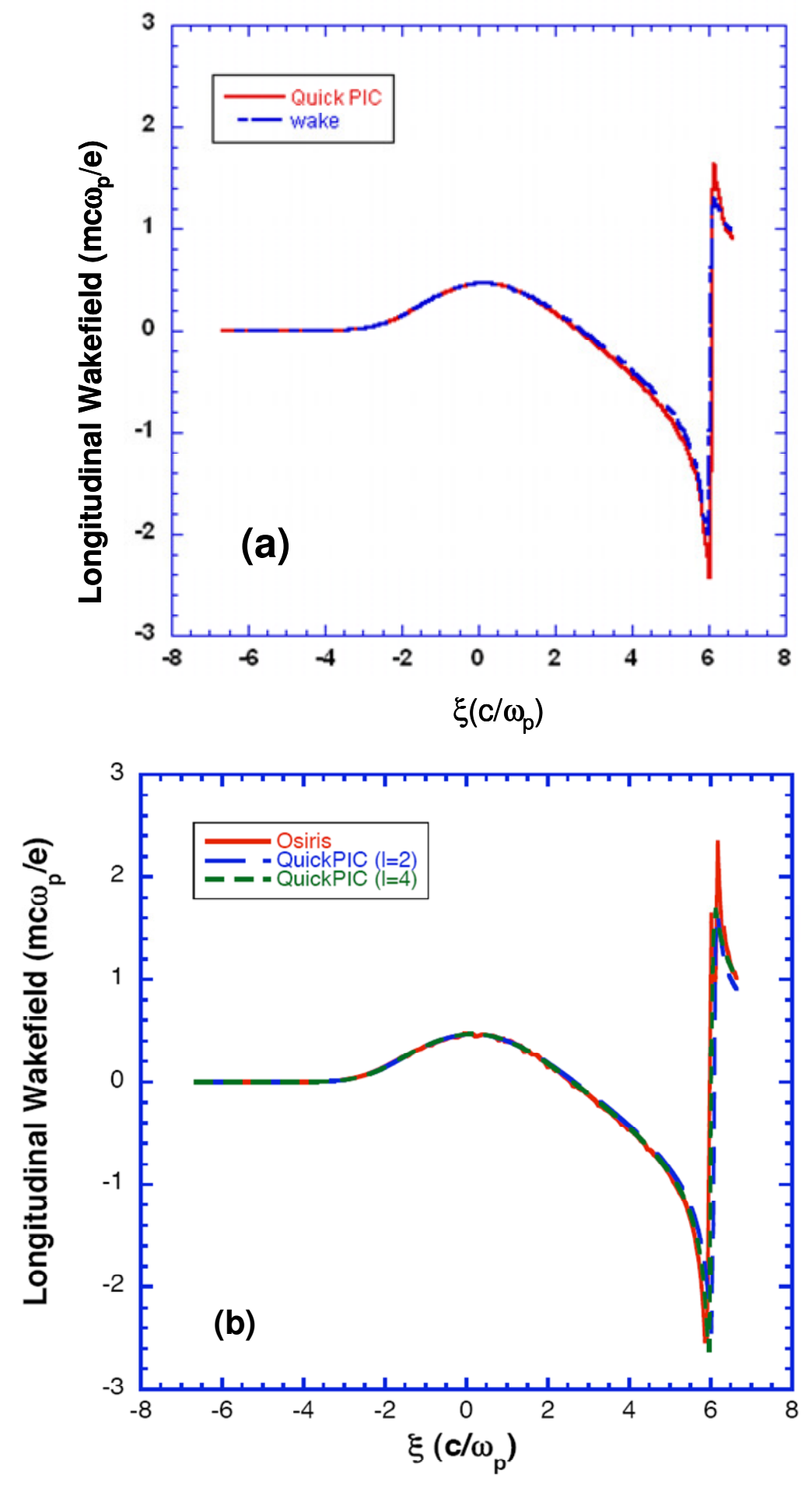

Fig.2.7) The comparison between longitudinal wakefield in OSIRIS and QuickPIC[9] ( $1=2$ and $\mathrm{l}=4$ are number of iterations) in part (a) and in part (b) the same result between WAKE and QuickPIC is depicted. The driver is moving from right to left in this plot. 


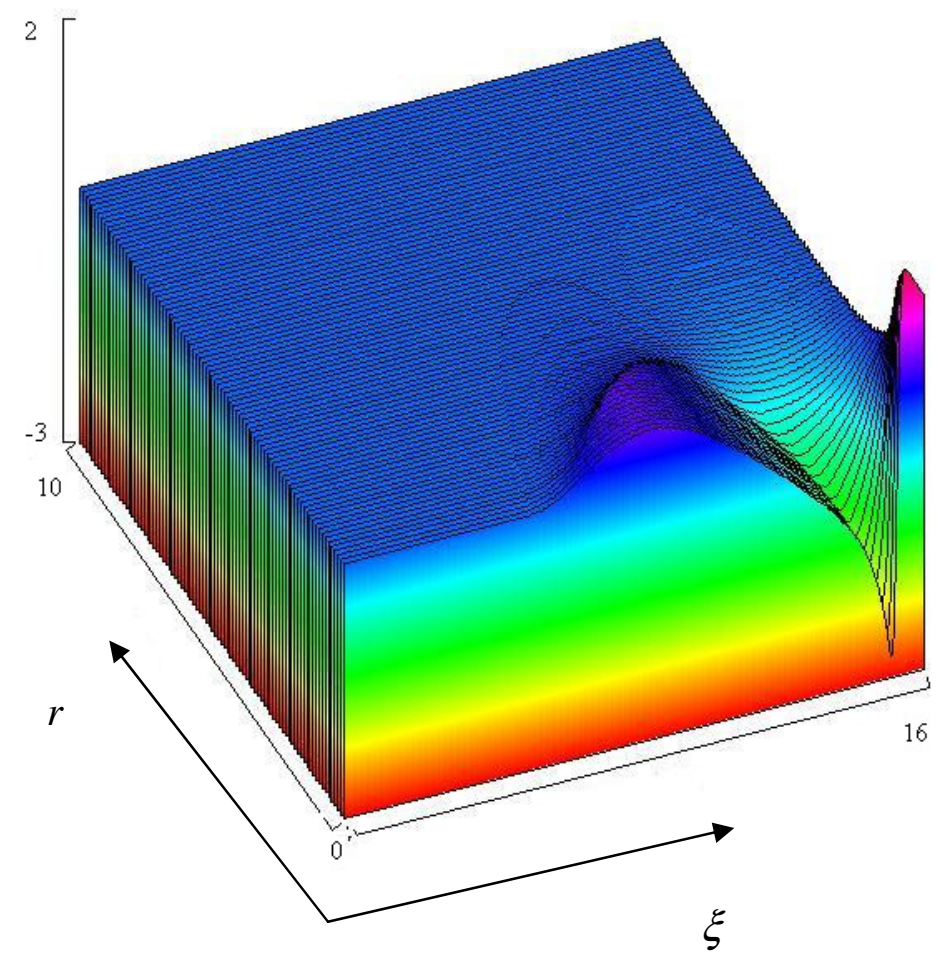

Fig.2.8) Surface plot of the wakefield from figure (2.7(a)). The vertical axis is longitudinal wakefield range of values like in figure (2.7(a)). 


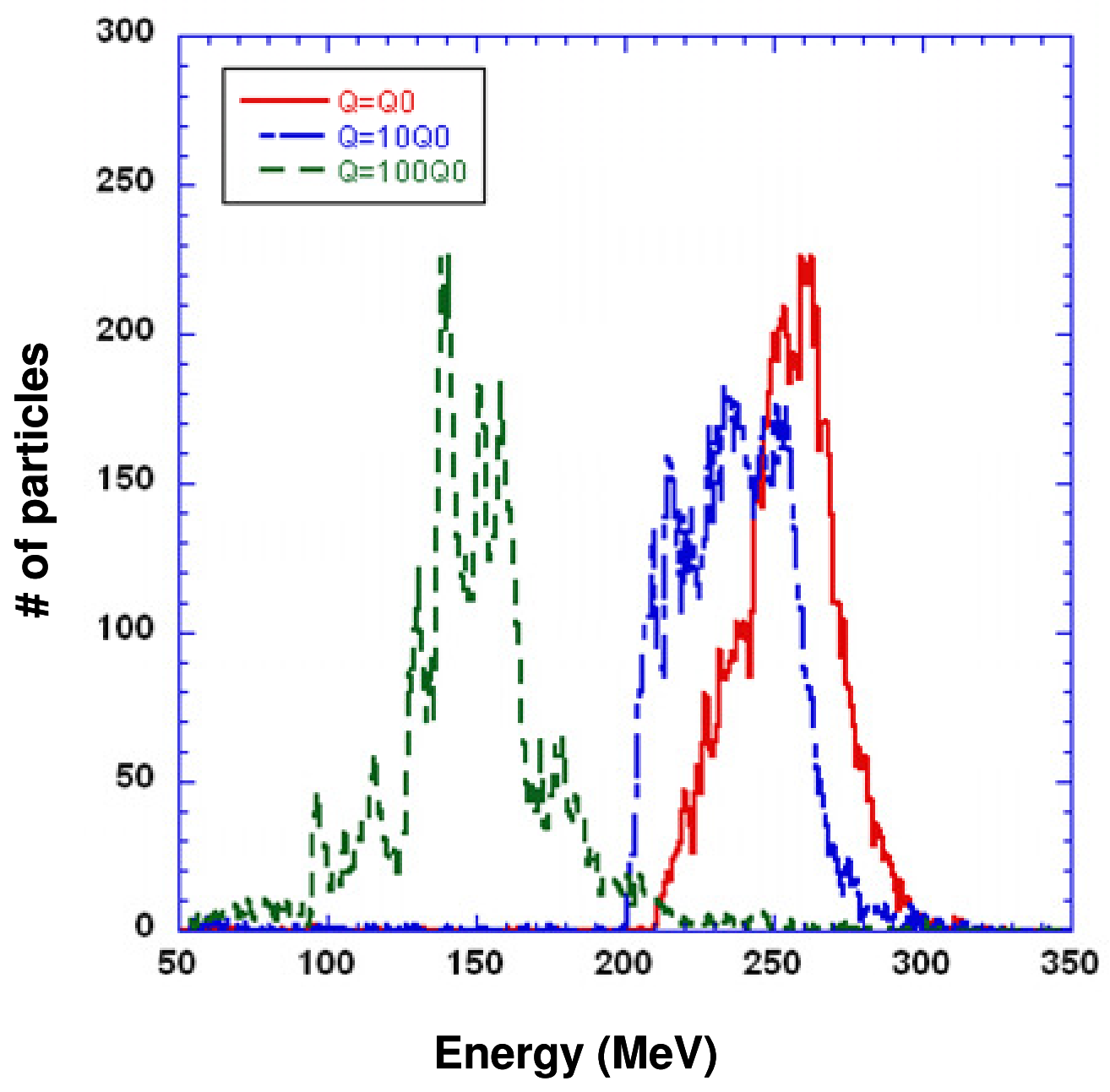

Fig.2.9) The effects of beam loading is investigated. $Q_{0}=2.88(n C)$ for red line and blue and green each increases by one order of magnitude with respect to each other. 


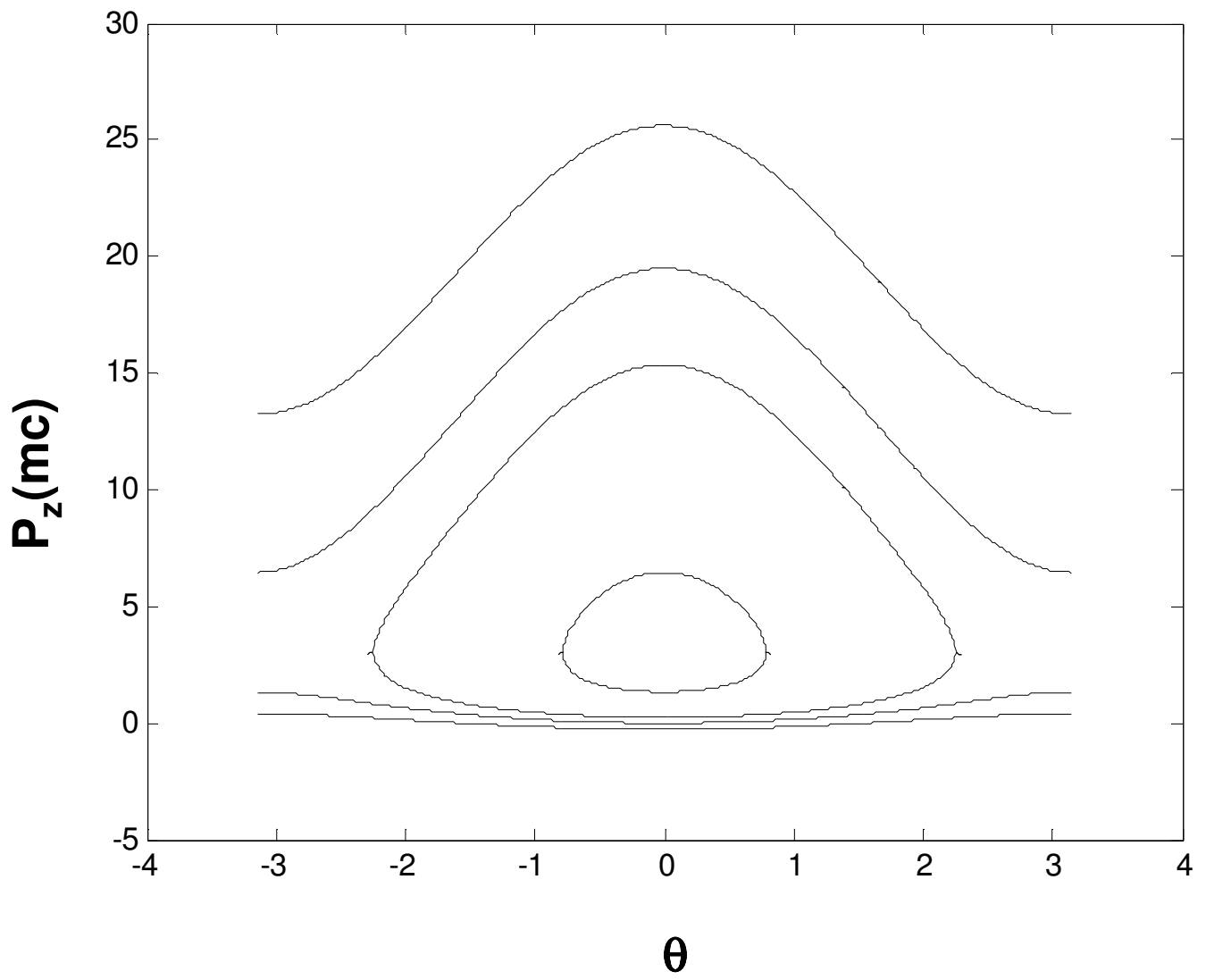

Fig.2.10) Plot of constant contours of Hamilton's equation. The close loops represent the trapped particles, while the open contours correspond to particles that one passes over by the wake. 


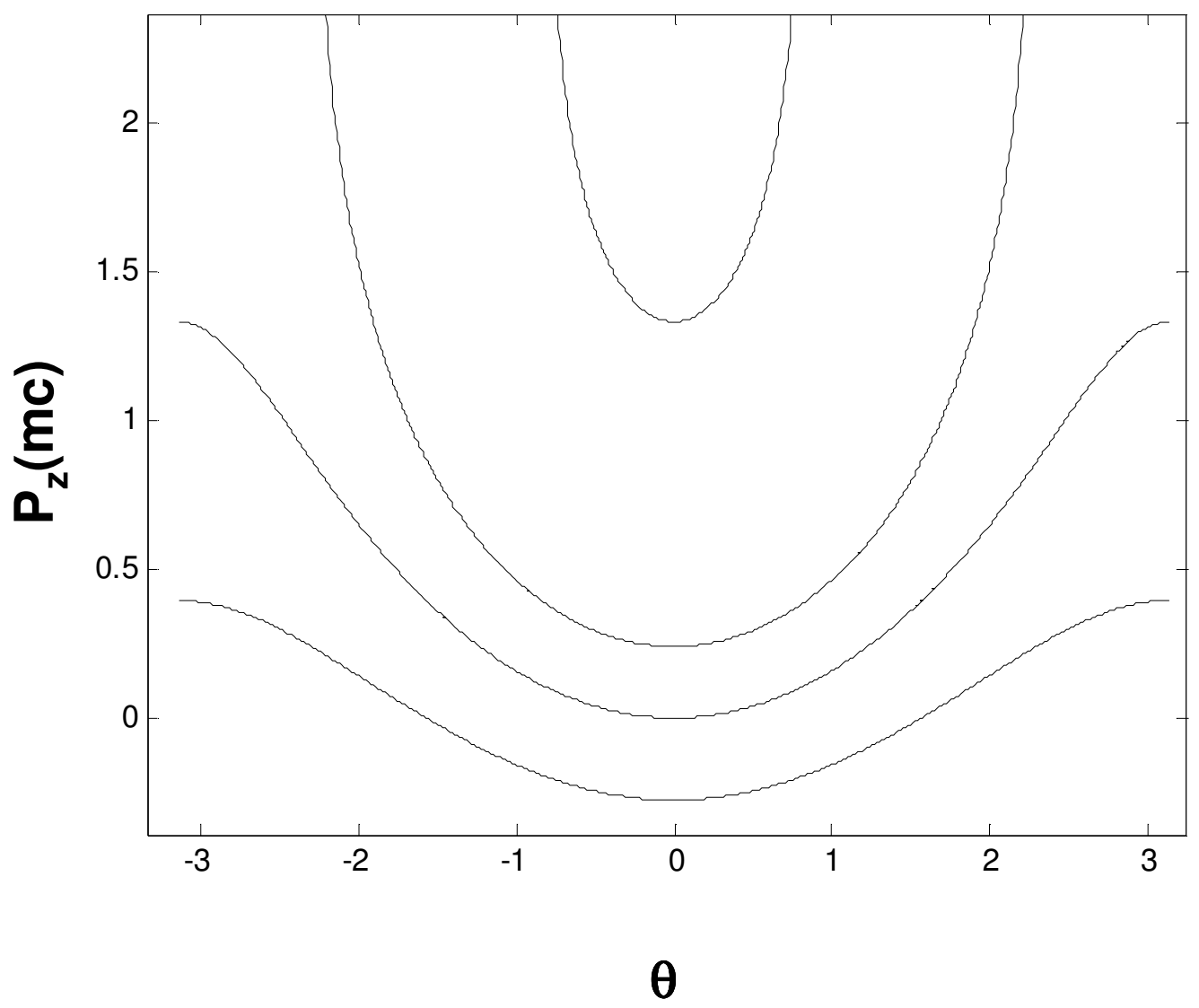

Fig.2.11) Blown up of the figure (2.10) about the region of interest (minimum $\mathrm{P}_{\mathrm{z}}$ ). 


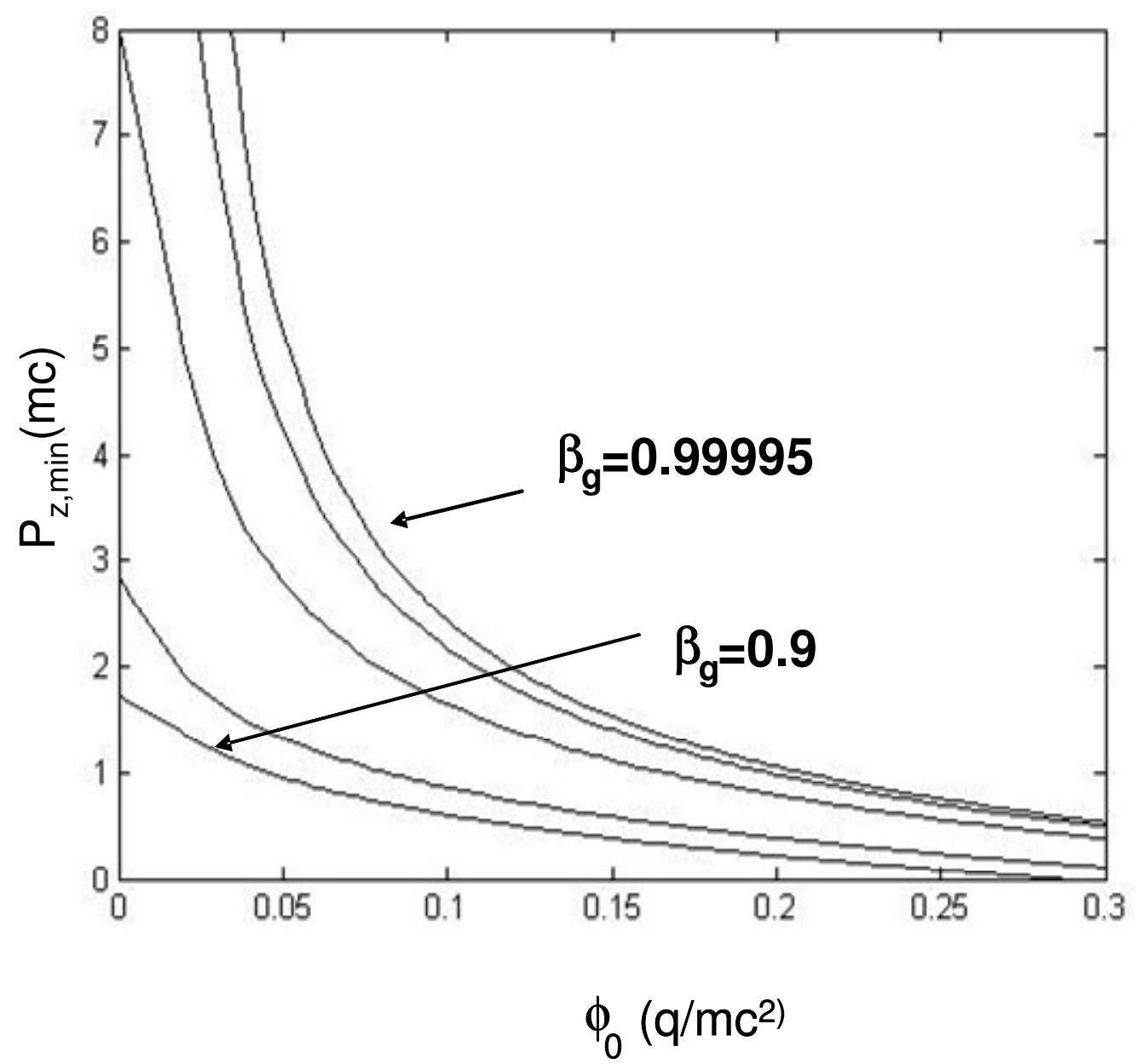

Fig. 2.12) The minimum required value for longitudinal momentum in $1 \mathrm{D}$ calculations is calculated and depicted with respect to potential amplitude. Each line represents different group velocity normalized to speed of light from the bottom 0.9 to $0.95,0.995,0.9995$ and finally 0.99995 and how it affects the trapping conditions. 

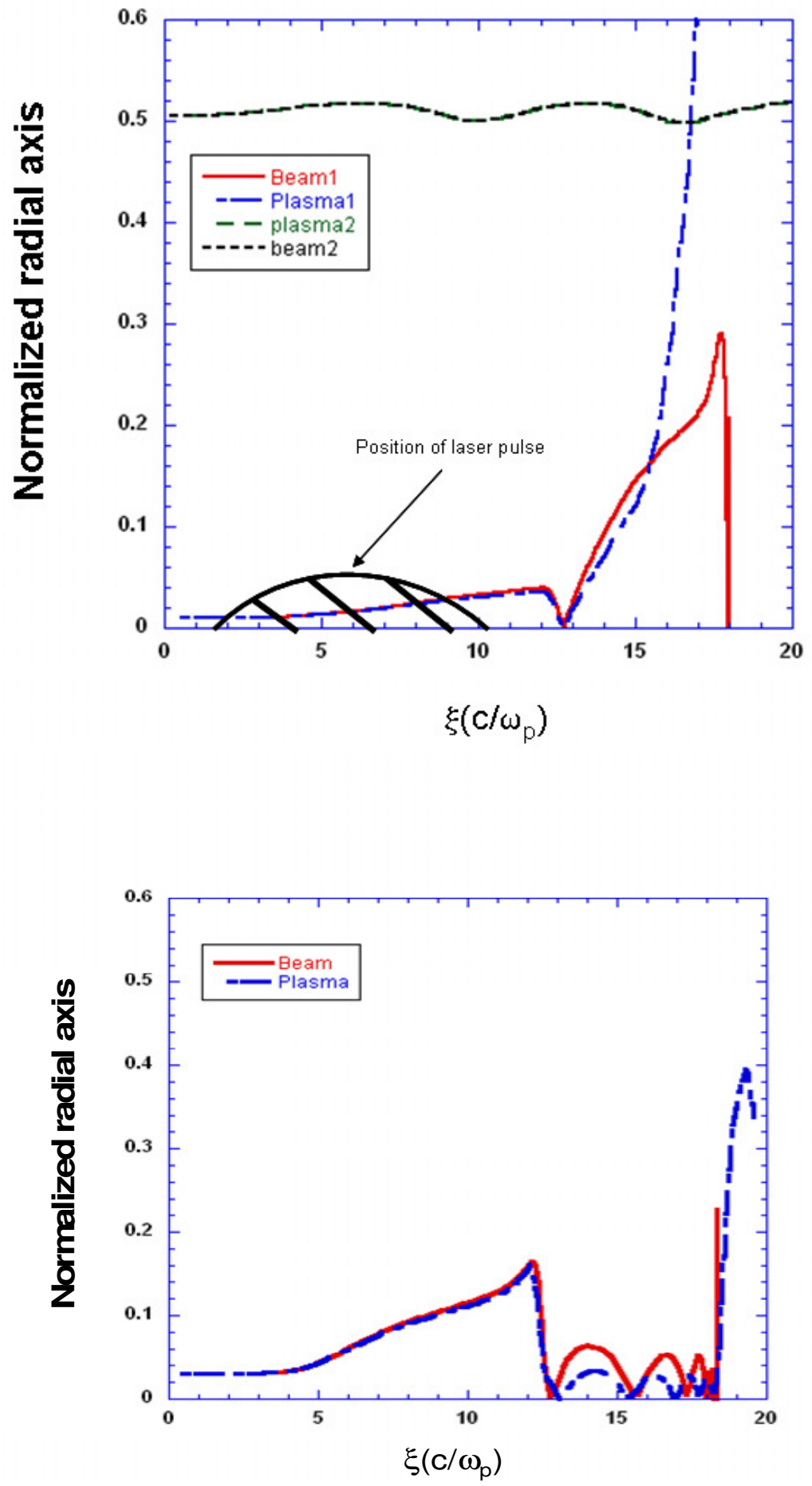

Fig.2.13) Two sets of trajectories are plotted (left). The particles enter the simulation from left and also position of laser is depicted Each set represents the same particle treated in two algorithms of plasma or beam particle. Plasma1 represents a particle which would become energetic but this algorithm can not satisfy the conditions for this particle and beam1 shows how the same particle would behave if treated correctly and after crossing the axis is trapped in the wakefield and oscillates around it. On the other hand the non energetic particles behave the same in both algorithms as depicted in plasma2 and beam2. The figure on the bottom follows another particle and a possible trajectory that this energetic particle has. 


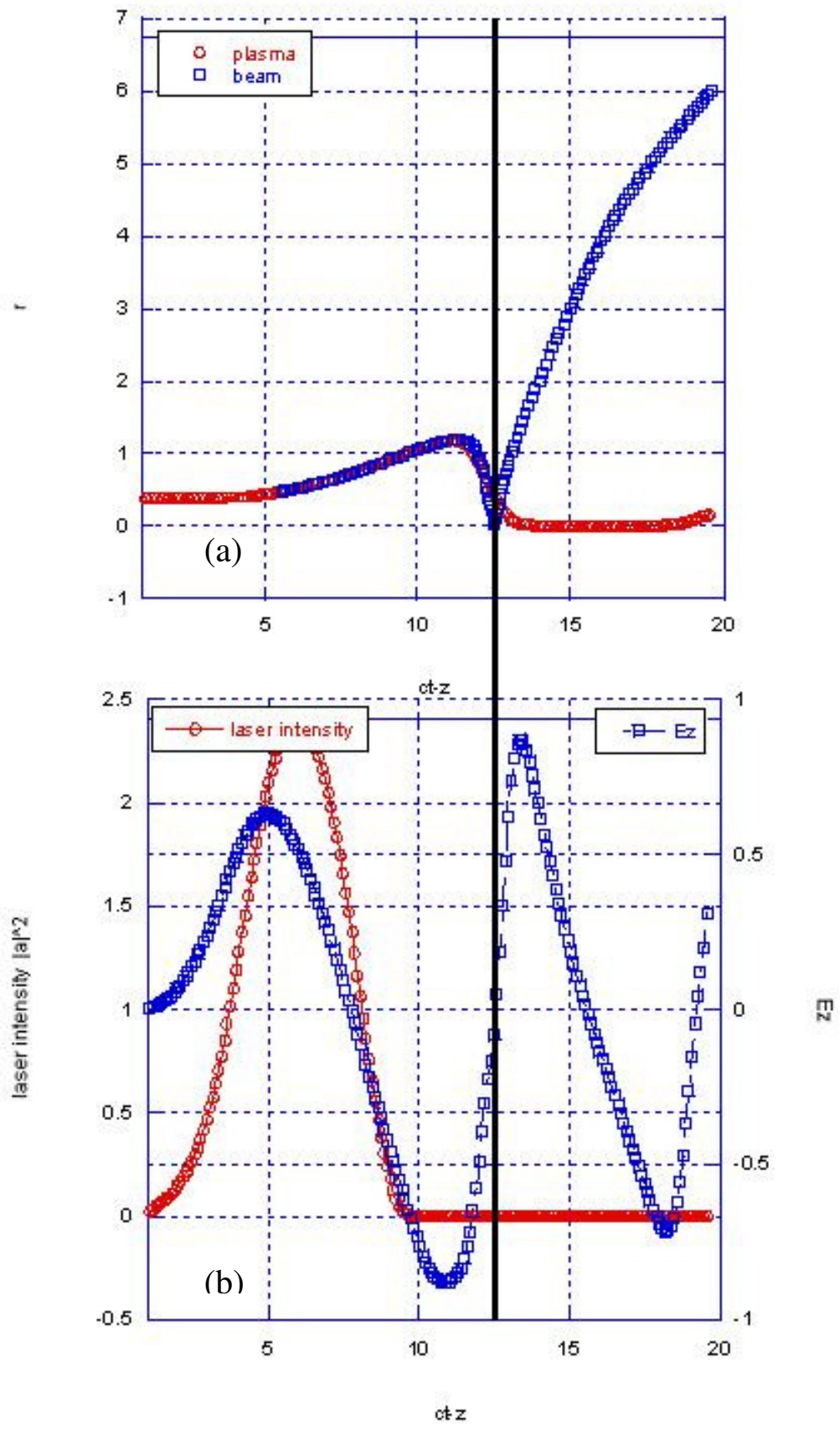

Fig.2.14) a) the trajectory of a plasma and a beam particle is shown. Although in this case the particle did not stay trapped and left the simulation as a beam, it is depicted to illustrate the exact position of laser pulse and more importantly the wakefield as the particles become trapped, which is shown in (b). 


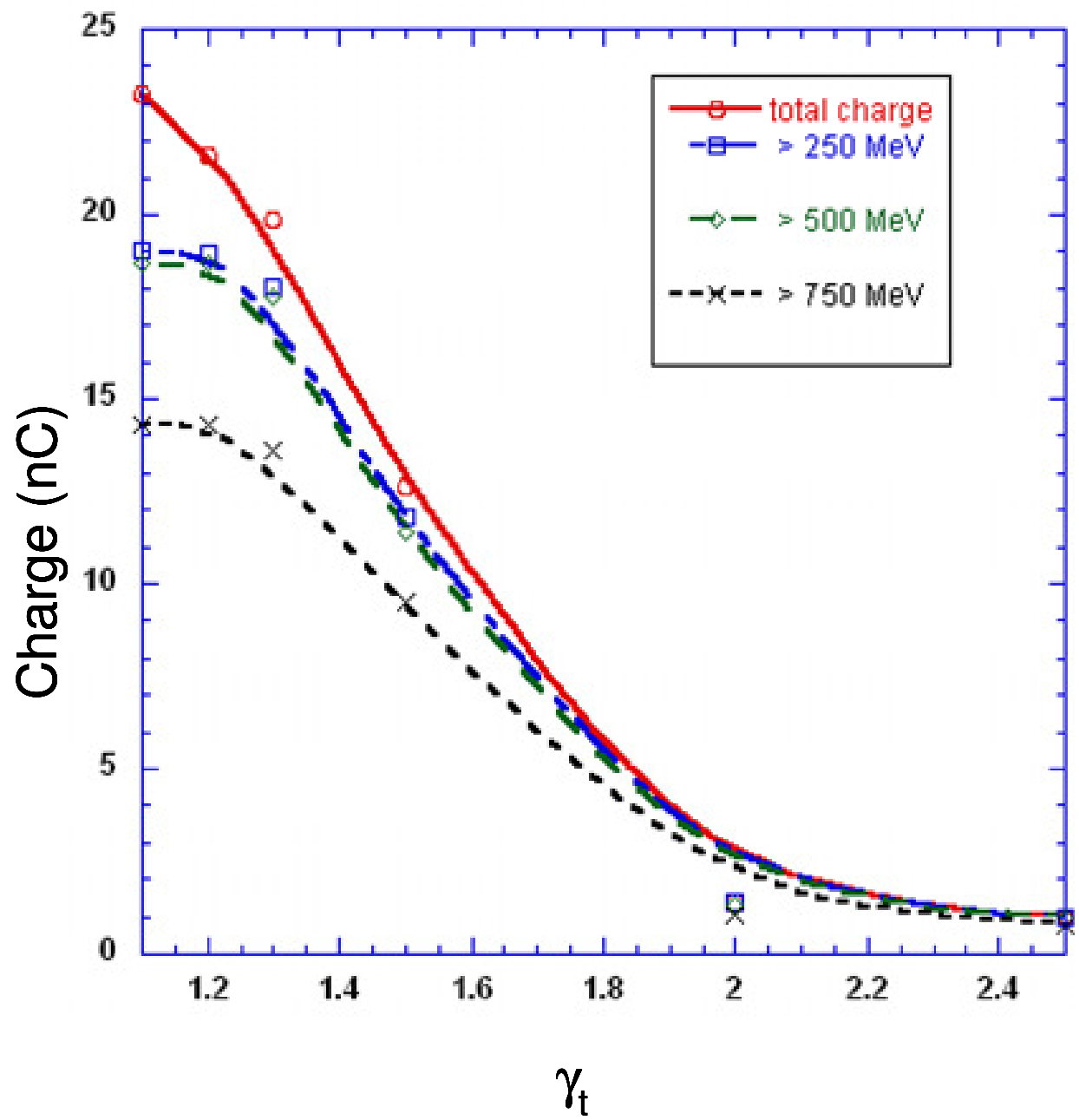

Fig.2.15) Investigating the change in $\gamma_{t}$ and its effect on the amount of charge which get accelerated to high energies where beam is passive. In this case number of particles with $E$ above 750,500 and 250 are shown. 


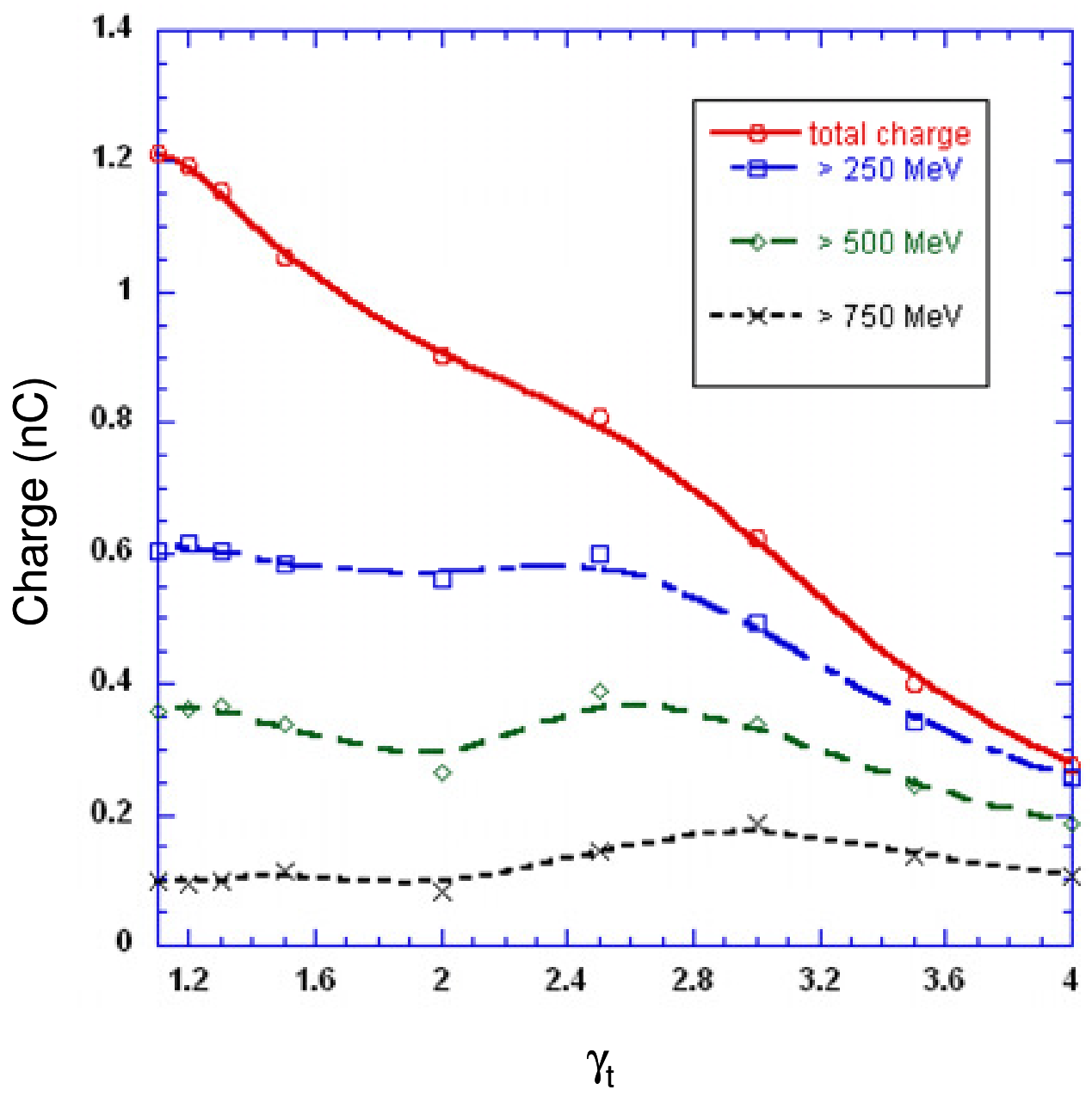

Fig.2.16) The same as figure 2.15 but with data which has self consistency resptored. 


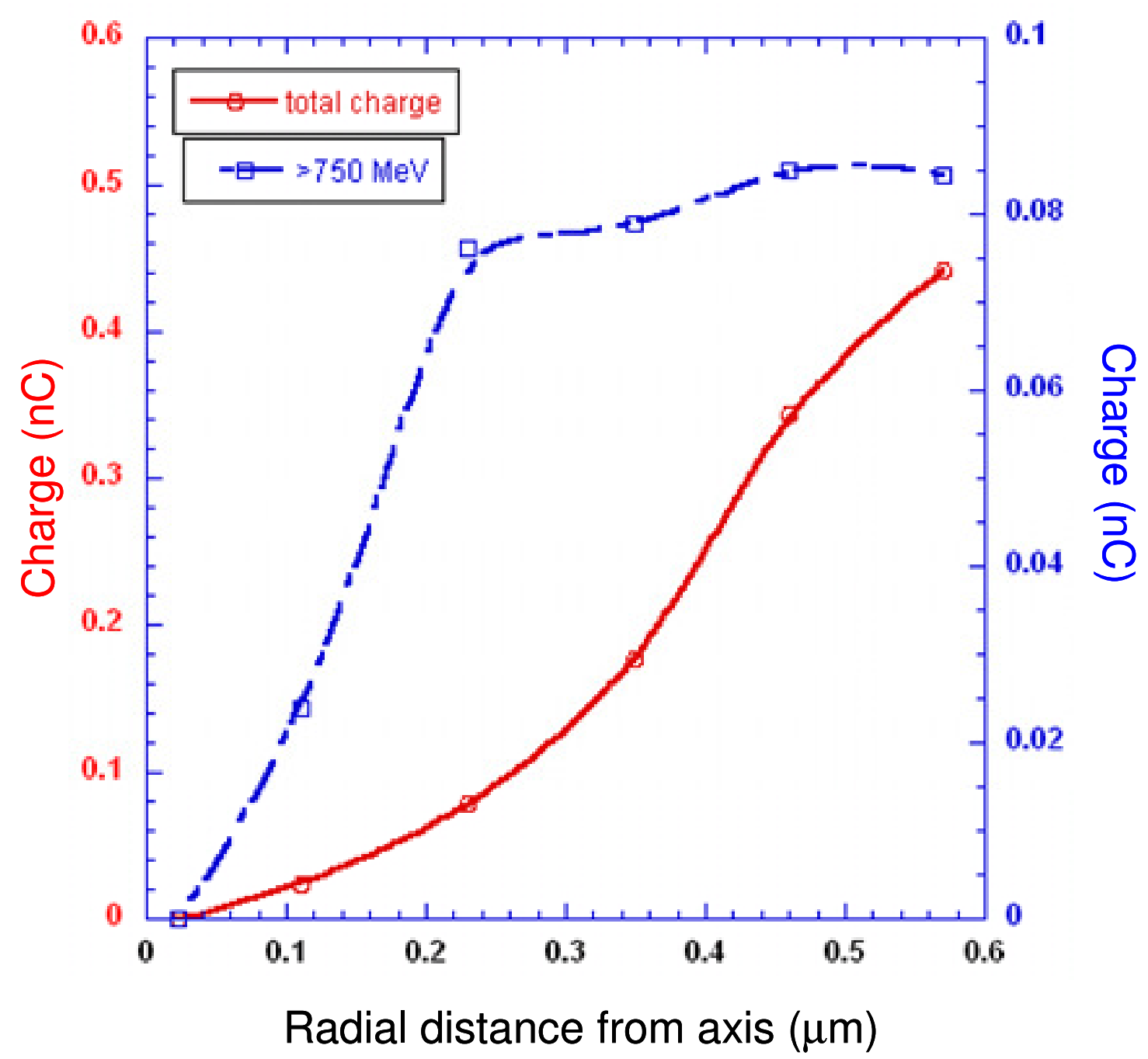

Fig.2.17) For the case of constant $\gamma_{t}=1.2$ the wave breaking condition is implemented by varying the distance from the axis which would promote the particle. In this plot particles which gained energies above $750 \mathrm{MeV}$ are shown. 


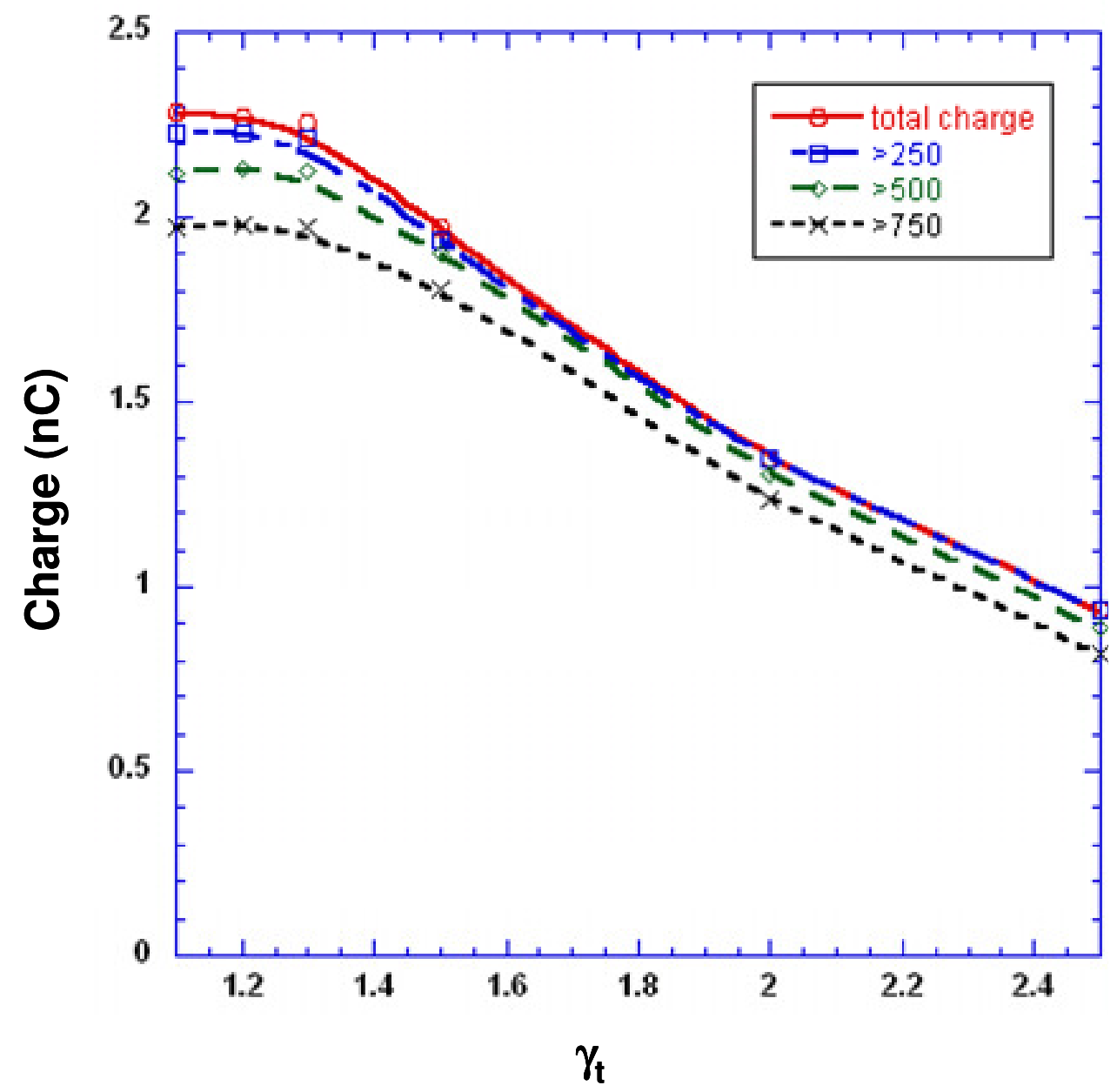

Fig. 2.18) Investigating the change in $\gamma_{t}$ and its effect on the amount of charge which get accelerated to high energies where wave breaking condition is also implemented in the passive beam case. In this case number of particles with $E$ above 750,500 and 250 are shown. 


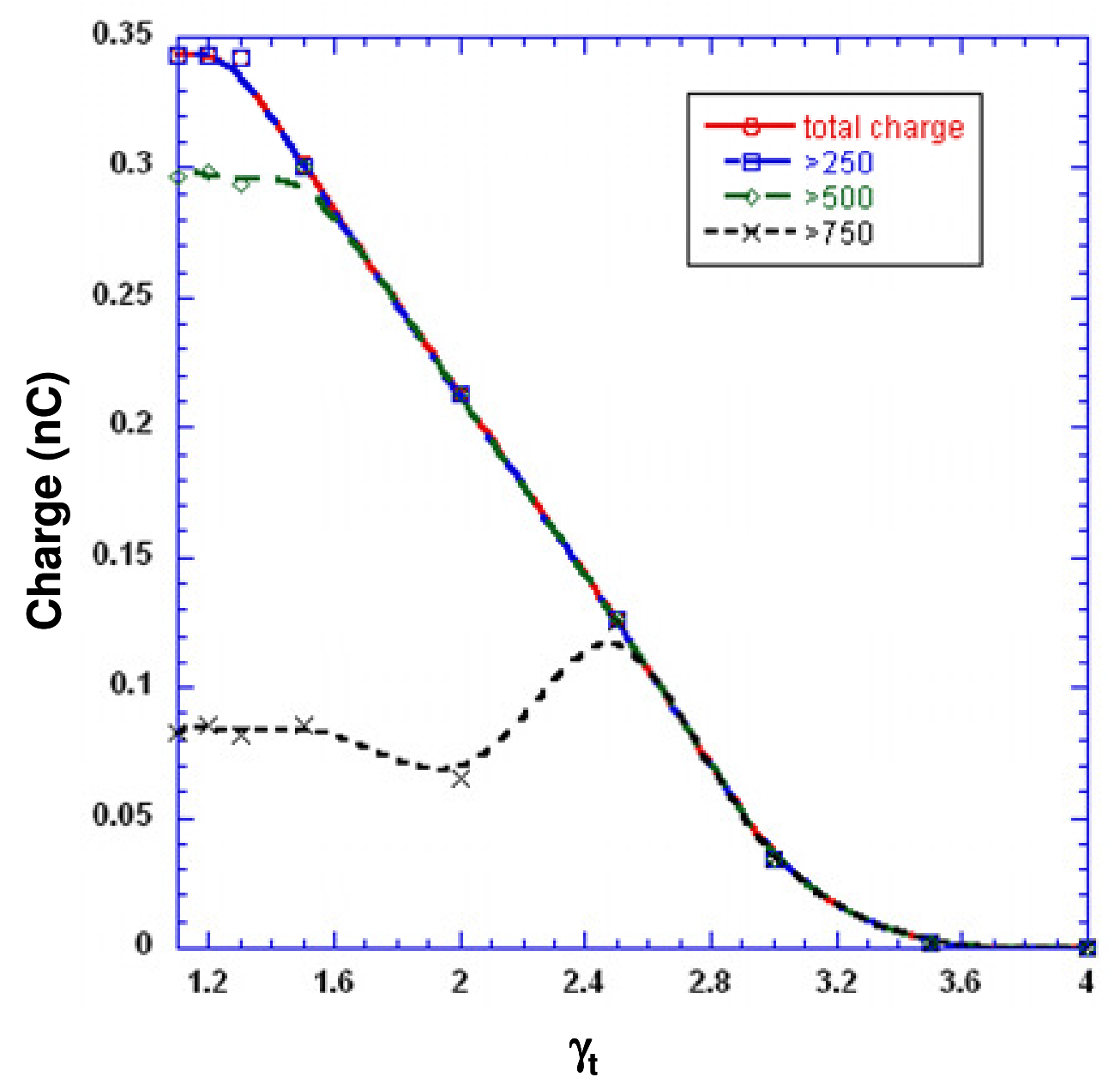

Fig.2.19) Investigating the change in $\gamma_{t}$ and its effect on the amount of charge which get accelerated to high energies where wave breaking condition is also implemented with selfconsistency restored. In this case number of particles with $E$ above 750, 500 and 250 are shown. 


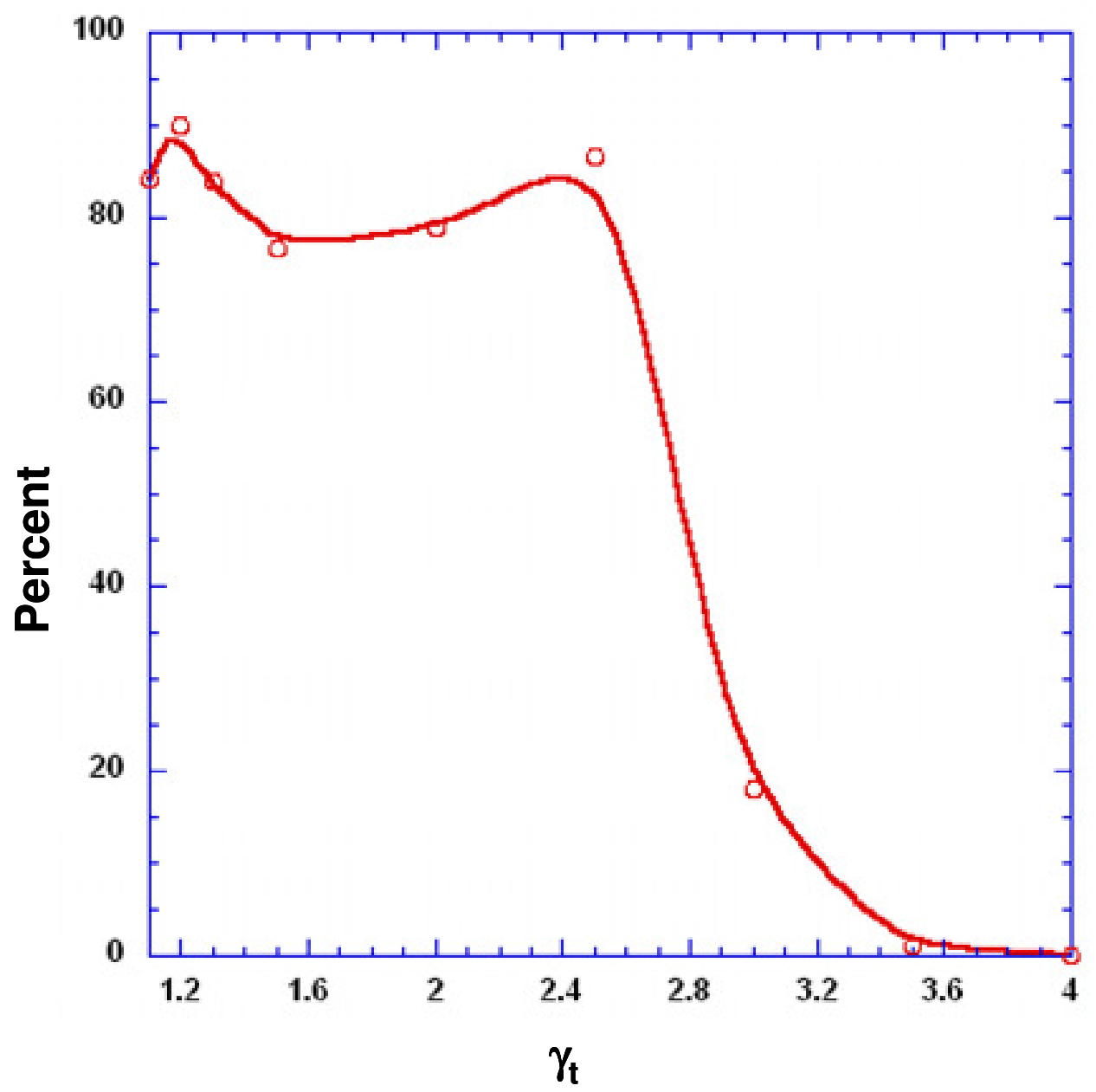

Fig.2.20) Taking the percentage of ratio of black lines (above $750 \mathrm{MeV}$ ) in figure 2.16 with respect to figure 2.19 . 


\section{Chapter 3: Comparing WAKE to OSIRIS simulation}

\subsection{The Conditions of OSIRIS simulation}

To benchmark our new WAKE code, we will compare our simulation with the OSIRIS code. This benchmarking is done in conditions which the original WAKE could not handle even for short distances. The reason being, in this case a very powerful laser (200 TW) is used and from the beginning of the simulation high energy particles are produced which should be promoted to beam status.

W.Lu et al. [4] have done full 3D OSIRIS simulations near the complete blowout regime. This is when the ponderomotive force or the radiation pressure of the ultra short laser pulse expels all the electrons from the path of propagation of laser over the interaction distance. The laser pulse propagates for many Rayleigh lengths without significant diffraction, because of total electron cavitation. The result of cavitation is a channel that guides the laser pulse.

The simulation is done for a $0.8 \mu \mathrm{m}$ wavelength, $200 \mathrm{TW}, 30$ fs laser pulse with

spot size $r_{s}=19.5 \mu m$ in a fully ionized $n=1.5 \times 10^{18}\left(\mathrm{~cm}^{-3}\right)$ plasma with the total axial length of close to $0.75 \mathrm{~cm}$. In this case the laser spot size is matched to the maximum blow out radius to create the best spherical wakefield. 


\subsection{Comparison of the results}

First we start by comparing the charge density distribution of the two codes. Figure (3.1) shows color images of the electron density in the $r-\xi$ plane for the two codes after $0.3 \mathrm{~mm}$ of propagation. The upper half of the image is the OSIRIS result and the lower half of the image is the WAKE result. (the WAKE result has been inverted so that it can be compared with OSIRIS result.) The electron density shows three regions of cavitation (black region), and the OSIRIS and WAKE simulations are in good agreement as to the period and shape of the cavitated regions. The bright filaments are high electron density regions where energetic beams of electrons are produced.

Figure (3.2) compares the longitudinal wakefield $\mathrm{E}_{\mathrm{z}}$ along the $\xi$ axis at $r=0$ for the two simulations (and after $0.3 \mathrm{~mm}$ of propagation). Recall, the laser is propagating to the right in these plots. Plotted are the electric fields from OSIRIS as well as the electric field from WAKE using two different promotion schemes. In figure (3.2) the red curve shows the simulation when only the trapping threshold $\left(\gamma_{t}=1.2\right)$ has been used to promote particles. The blue curve shows the electric field when both the energy threshold $\left(\gamma_{t}=1.2\right)$ and the wave breaking criteria $\left(r_{w b}=0.65(\mu m)\right)$ are used.

In this case the 200TW laser is too powerful and from the beginning of the simulation we need to promote the plasma particles which can not be treated with the quasi-static approximation. This causes a large number of particles being removed from plasma calculations and although many of these particles may not become trapped in order for the governing equations of the code to be valid they have to be promoted. As 
mentioned before this causes us to account for the transverse current density of these

promoted particles in the calculations $j_{\perp}=j_{\perp}^{\text {plasma }}+j_{\perp}^{\text {beam }} \cdot$

The implementation of wave breaking condition can address this issue without increasing the number of time steps. The blue curve in figure (3.2) shows how the addition of the wave breaking condition has improved the result. It should be noted that because we promote fewer unnecessary particles, the code also runs more efficiently. So the blue curve in figure (3.2) shows a much better agreement with the full PIC code, which is a very remarkable result.

A final comparison is done with the energy spectrum of the accelerated particles after $7.5 \mathrm{~mm}$ propagation in the plasma. Figure (3.3) shows two isolated spikes in energy spectrum using normalized units, from OSIRIS simulations. Each spike represents the first and the second bubble in the density profile. One with nearly $0.3(\mathrm{nC})$ of charge around $1.5(\mathrm{GeV})$ and the other with $50(\mathrm{pC})$ charge at $700(\mathrm{MeV})$.

WAKE results in figure (3.4) shows the same maximum spike at $1.5(\mathrm{GeV})$ and also small bucket around $700(\mathrm{MeV})$ the total charge under the curve is about $0.25(\mathrm{nC})$ which is smaller than OSIRIS and can be attributed to the fact that by trapping only the particles which were crossing the axis some of the potentially energetic electrons were ignored. Still the simulation accurately shows the energy spectrum. The other point is that this code is capable of doing long channel simulations in very short times which is nearly impractical to do on 3D full PIC codes. 


\subsection{Summary of the chapter}

In this chapter another comparison to validate the results from WAKE simulation is done. The results from WAKE a 2D cylindrically symmetric code with the OSIRIS full PIC code in 3D are evaluated. We found very good agreements between our results. The fact that after $7.5 \mathrm{~mm}$ of propagation the results are very comparable is remarkable since the process of promotion of particles and consequently omission of them from background plasma is a noise generating process. Even with all the provisions we make to minimize the noise it accumulates as the simulations advances through the length of the channel.

It needs to be noted, all the simulations in this chapter (and the whole thesis) has been done on a single desktop computer with 1 processor in the matter of hours, in comparison with the 3D codes which need cluster of CPU's and days of calculations. 


\subsection{Figures}

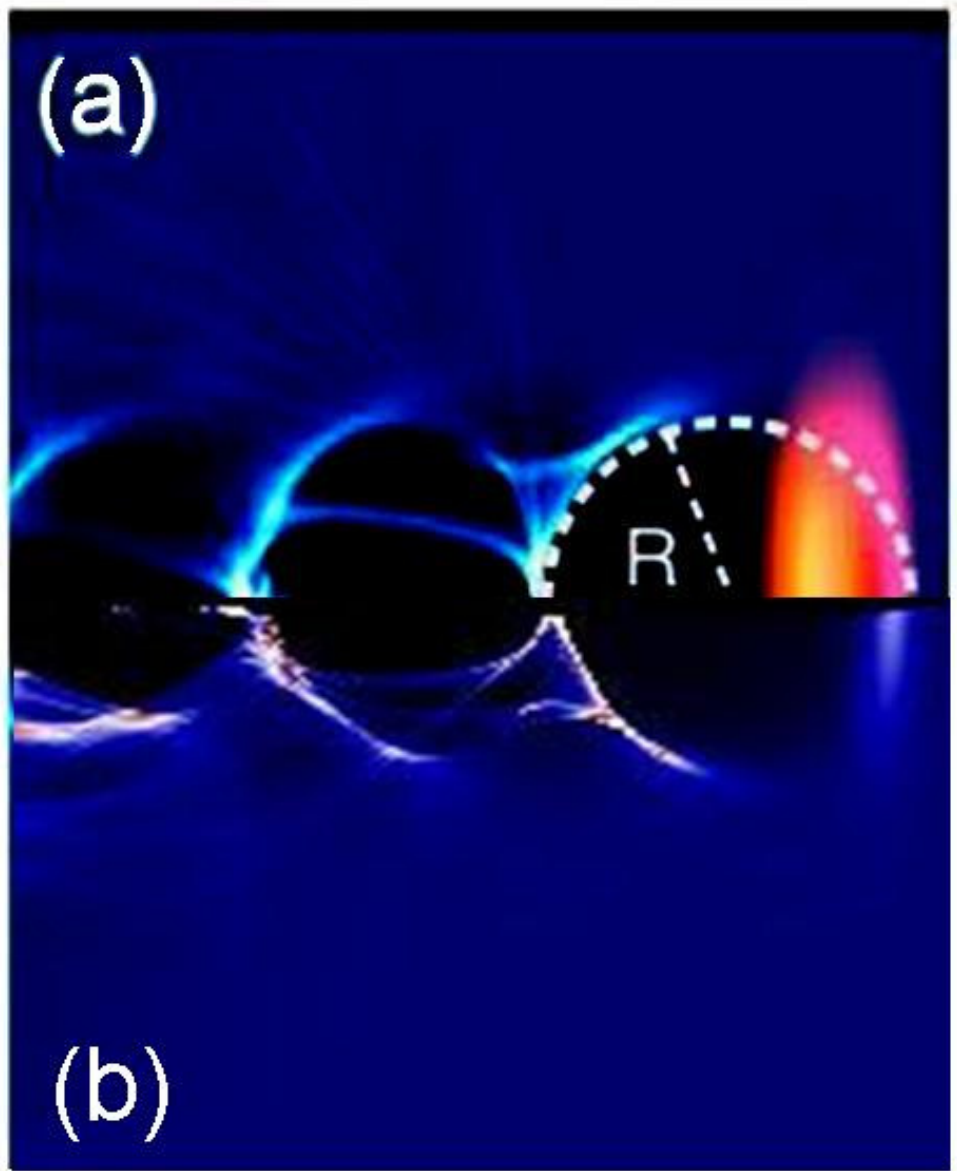

Fig.3.1) (a) shows OSIRIS simulation [2] after $z=0.3 \mathrm{~mm}$ propagation. The laser pulse propagating to the right is depicted in orange. (b) shows the same simulation using WAKE and clear buckets that are formed. 


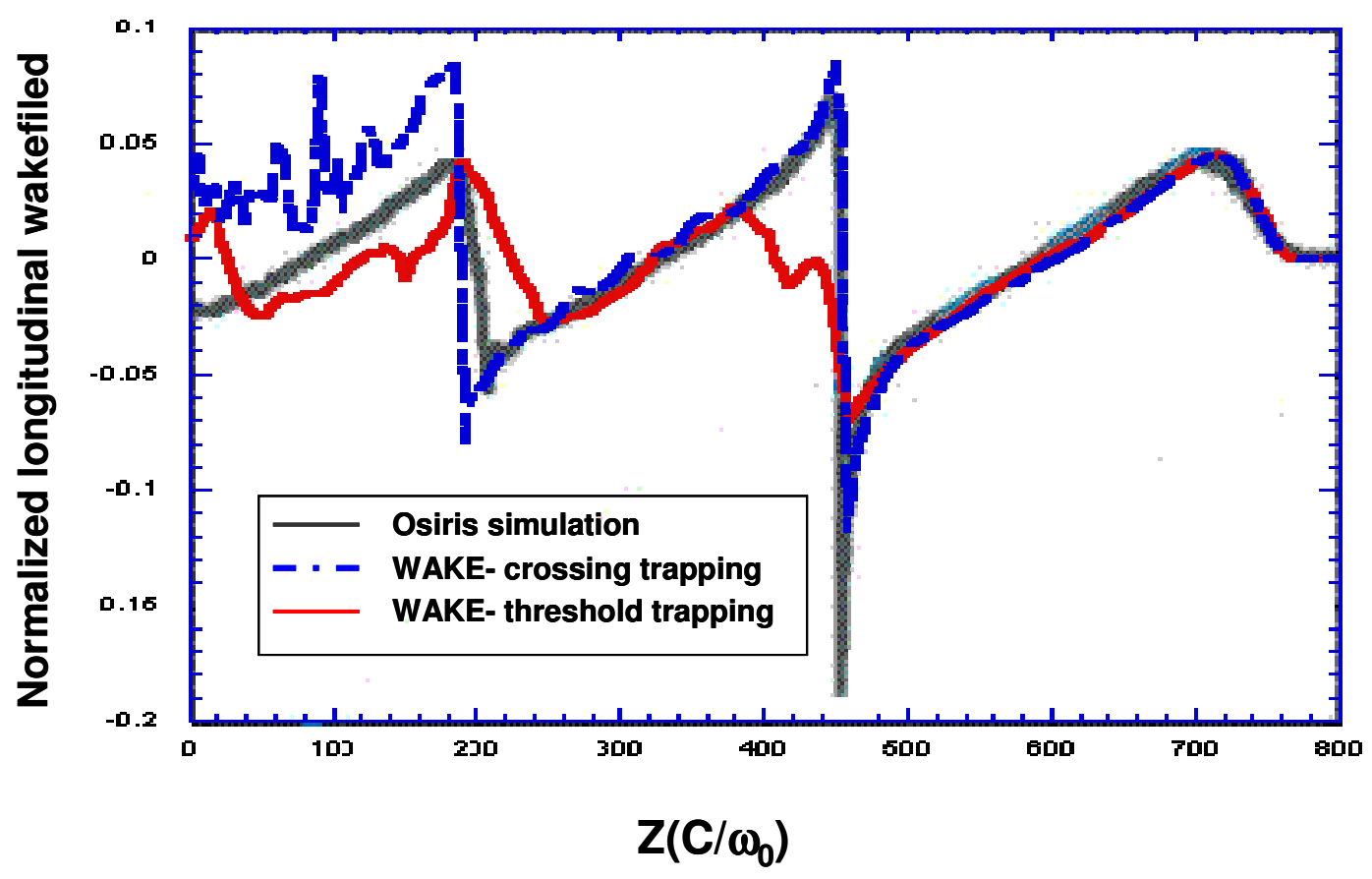

Fig.3.2) The gray curve is $E_{z}$ after $0.3 \mathrm{~mm}$ propagation with OSIRIS (the benchmark). Red shows WAKE simulation of the same conditions using only threshold trapping and blue shows addition of wave breaking condition to the promotion algorithm. 


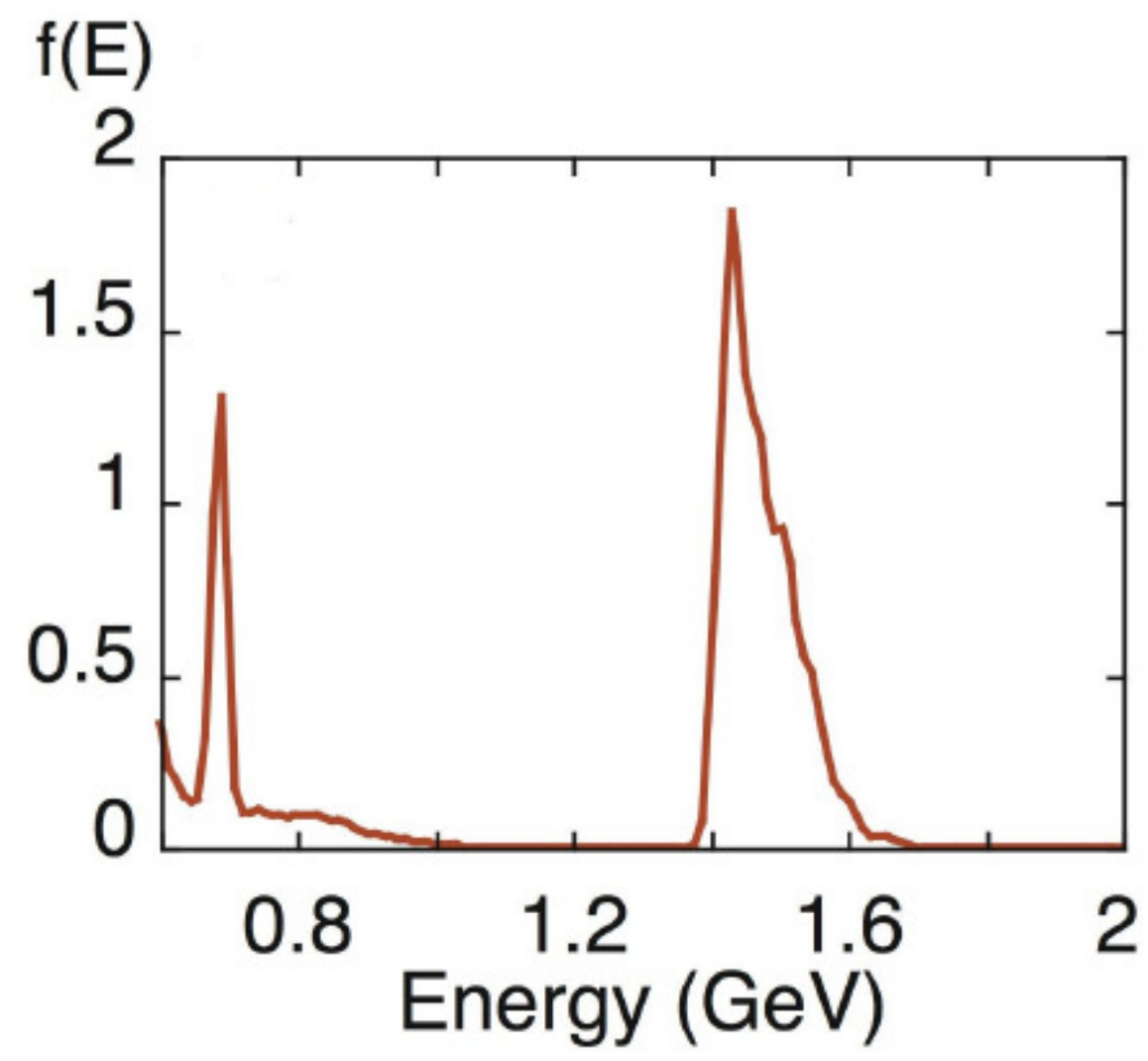

Fig.3.3) The energy spectrum after $7.5 \mathrm{~mm}$ propagation using OSIRIS and the peak energy power around $1.5 \mathrm{GeV}$ 


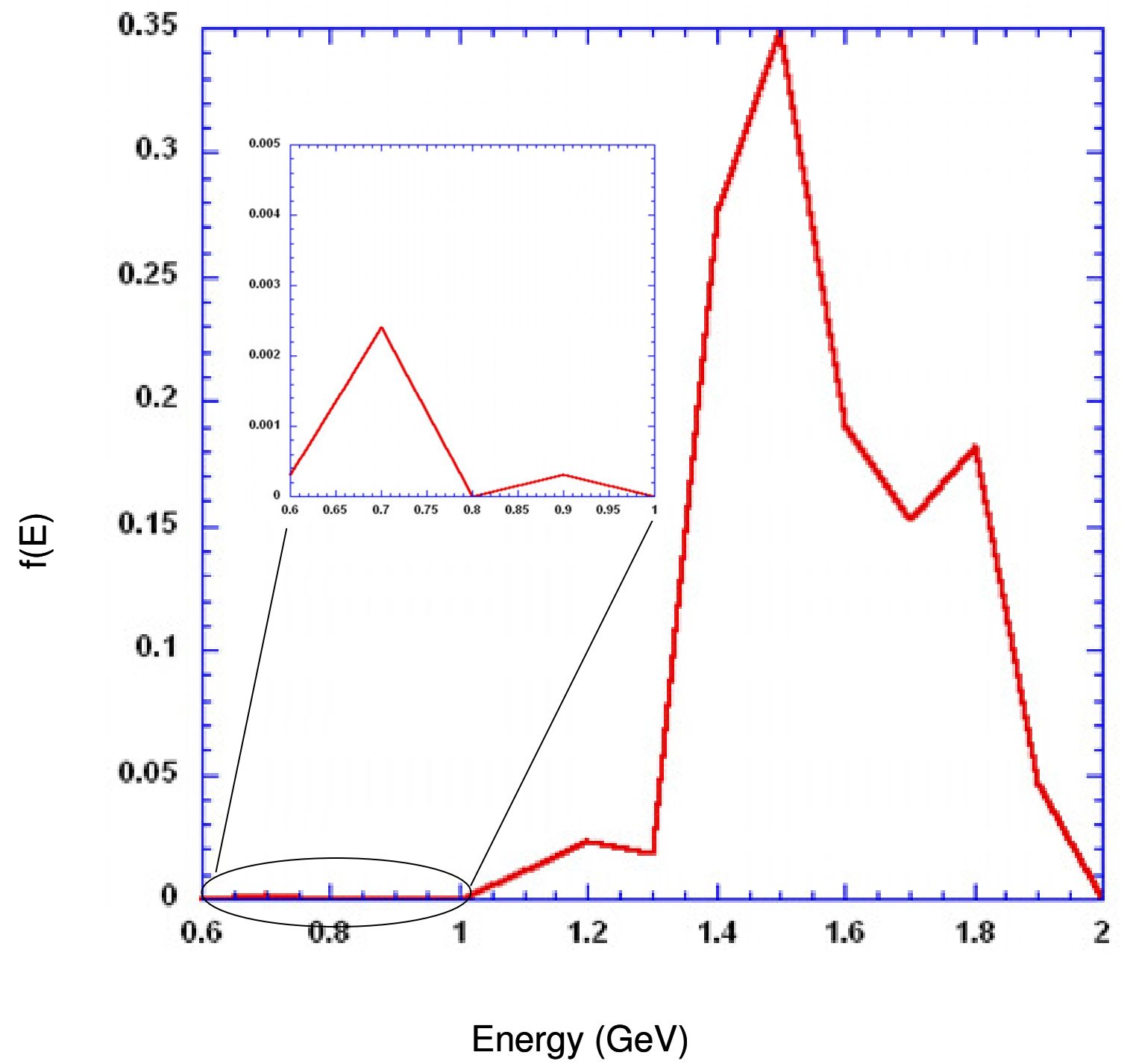

Fig.3.4) The same simulation as figure 3.3 with WAKE and showing the same principal behavior as OSIRIS. 


\section{Chapter 4: Simulation of LNBL experiment for $1 \mathrm{GeV}$ electron beam}

\subsection{The experiment}

In order to achieve laser driven $\mathrm{GeV}$ acceleration two methods are proposed:

1) Using very powerful lasers in petawatt (PW) scale with large sport size to increase the Rayleigh length $\left(z_{R}=\omega_{l} r_{s}^{2} / 2 c\right)$ in the uniform plasma channel.

2) Use channel guided laser beam in a centimeter scale channel with less power, in about ten's of terawatt (TW).

In the first case the propagation is limited to Rayleigh range and only self focusing can increase the interaction length. Even self focusing because of erosion of leading edge of laser is limited. By increasing the spot size and effectively the Rayleigh range the laser power should increase to petawatt scale.

The channel guided method with today's laser technology is the more practical approach. Even in the channel guided mode, one has to note that the dephasing length will limit the length that particle can be accelerated. This happens because as the particles become relativistic and the wake is travelling with the phase velocity $v_{p}<c$ eventually the particles will outrun the wake and move into decelerating phase. Experiments [22-24] 
have shown when the accelerating length was matched to the dephasing length it lead to low energy spread electron beam production.

Leemans et al. [3] reported the first observation of $\mathrm{GeV}$ electron beam acceleration from centimeter scale experiment using a gas-filled capillary discharge waveguide in 2006. With laser pulse energy of 40 TW they produced a nearly mono energetic beam of about $30(\mathrm{pc})$ of electrons accelerated to $1 \mathrm{Gev}$. Figure (4.1) shows e-beam spectra of the capillary guided accelerator. In his figure 2 electorn beams are visible the bright one at $1 \mathrm{GeV}$ represents the main acceleration of particles and the second one at around $0.8 \mathrm{GeV}$ which shows the secondary acceleration of electrons.

\subsection{Using Externally injected particles to simulate the experiment}

In our first simulations of this experiment we injected externally the beam particles to determine the possible energies that could be achieved (at this time we do not promote plasma particles, only injected particles are used). The electron beam parameters such as width, length and charge of the bunch were varied and compared with experiment. The laser parameters are given in table 4.1 .

The beam was taken to have a spatial distribution that was Gaussian radially, and flat longitudinally. The simulation was done with 10,000 (nearly 3 (nC) of charge) beam particles. Figure (4.2) shows the placement of particles in the wakefield created by laser. This place has been shown theoretically to be where most of the trapping occurs.

We can now plot the distribution of these particles after propagation in the capillary and examine the possibility of achieving $1 \mathrm{GeV}$ acceleration with the presented parameters. The histogram in figure (4.3), shows the number of particles which are 
accelerated to $1 \mathrm{GeV}$ energies after about $1.4 \mathrm{~cm}$ propagation in capillary This plot shows that a wakefield sufficient to accelerate electrons to a $\mathrm{GeV}$ is created by the laser pulse in the simulation.

\subsection{Self-trapping using only threshold trapping condition}

Our next step was to use the promotion criteria defined in chapter 2 to simulate the self-trapping process in the LNBL experiments. In this simulation the parameters of LNBL experiment that are implemented in WAKE are listed in table 4.1.

\begin{tabular}{|c|c|}
\hline Capillary diameter & $310(\mu \mathrm{m})$ \\
\hline Laser spot size & $\mathrm{r}_{\mathrm{s}}=25(\mu \mathrm{m})$ \\
\hline Quadratic plasma density profile & $\mathrm{n}=\mathrm{n}_{0}+\Delta \mathrm{n} \mathrm{x}_{\perp}[\mu \mathrm{m}]^{2}$ \\
\hline Electron plasma density & $\mathrm{n}_{0}=4.3 \times 10^{18}\left(\mathrm{~cm}^{-3}\right)$ \\
& $\Delta \mathrm{n}=2.9 \times 10^{14}$ \\
\hline Laser pulse length & $\tau=40(\mathrm{fs})$ \\
\hline \#plasma particles per cell in simulation & 4 \\
\hline
\end{tabular}

Table 4. 1) Parameters which used to simulate LNBL experiment with WAKE.

Figure (4.4) shows after $1.4 \mathrm{~cm}$ propagation in the capillary the energy distribution of particles versus their position in $\xi$. For this simulation the energy threshold was $\gamma_{t}=2.5$. Figure (4.4) shows particles accelerated to near $1 \mathrm{GeV}$ energies 
but the energy distribution is far from monoenergetic, which is also shown in the histogram of energy of particles in figure (4.5). We notice from figure (4.4) that the promotion process does not stop even after long distance of traveling of the laser pulse in the capillary. Since, after $1.4 \mathrm{~cm}$ propagation in capillary there are still very low energetic particles in the simulation as it can be seen from the histogram in figure (4.5).

To advance this point further, the same simulation is done for $\gamma_{t}=4.5$ with the exact conditions as before to evaluate if we can get near monoenergetic particles. As it can be seen from figure (4.6) and its histogram in (4.7) the promotion process stops for these high values of threshold, since there is no very low energy particles remained in the simulation and the system is closer to having low energy spread. But these values for threshold are not justifiable according to theory presented in previous chapter.

Another point to consider is the amount of laser energy absorbed by the wakefield. Figure (4.8(a)) shows the laser field intensity at the beginning of simulation and figure (4.8(b)) shows the laser field intensity at the end of capillary, which shows pulse depletion and strong diffraction of the laser. In figure (4.9) the energy of laser pulse is plotted vs. the distance of propagation. We can see that energy of the laser pulse depletes very fast and after $1.5 \mathrm{~cm}$ propagation of laser it nearly reaches $10 \%$ of its total energy. These figures show the evolution of laser pulse and we have to follow these changes closely since as energy of the laser gets absorbed, $\omega_{l}$ becomes smaller and at some point the conditions for validity of envelope approximation $\omega_{l}^{2}>\omega_{p}^{2}$ deteriorates. In our simulation by examining for different axial number of grid points (200 to 400 and then to 800 points) we did not see any change in our results, suggesting we have not approached these limits. 


\section{$\underline{4.4 \text { Self-trapping with implementation of all trapping conditions }}$}

With the implementation of both the energy threshold and the wave breaking condition, we have simulated the LNBL experiment.

Figure (4.10) shows the particles energies versus their position in $\xi$. These results are obtained with $\gamma_{t}=1.2$ and radius for wave breaking of $r_{w b}=0.46(\mu m)$ at time $t=4.25 t_{R}$ (or distance $1.4 \mathrm{~cm}$ ) where $t_{R}$ is the Rayleigh time based on a $25(\mu \mathrm{m})$ spot size. It can be seen there are two buckets of accelerated electrons. The first one corresponds to the self trapped electrons in the first accelerating bucket and the second one, which has lower energy, corresponds to the second acceleration bucket. The energies of the electrons in the second bucket are lower, because they experience smaller electrical field in this bucket.

As one would expect when all the promotion conditions are present there are fewer particles that are promoted to beam particles, about $2.2(\mathrm{nC})$ as oppose to only threshold condition with nearly than $10(\mathrm{nC})$. Also after a while in the simulation there are no more plasma particles which reach the promotion conditions, because the wakefield is not strong enough to create such energetic particles, so the process of promotion of plasma particles to beam status stops. We have particles which have energies around $1 \mathrm{GeV}$ with smaller energy spread than those in the figure (4.4) where $\gamma_{t}=2.5$ 
To further investigate we can examine figure (4.11), which is the same as figure (4.10) only here $\gamma_{t}=2.5$ (the radius for wave breaking condition is also the same as figure (4.10). It shows the fact that at time $t=4.25 t_{R}$ there is no more trapping of new particles and more confinement of accelerated particles in physical space.

The histogram is plotted in figure (4.12) and shows that there are no particles left in lower energies. They are either accelerated near $\mathrm{GeV}$ energies or have left the simulation window. In this case the amount of charge around $1 \mathrm{GeV}$ is about $125(\mathrm{pC})$.

\subsection{Summary of the chapter}

In order to benchmark new WAKE we need to compare it to some experimental results in addition to other simulations. In this chapter we did implement the LNBL experimental conditions to best of our knowledge. We can never be sure that we have all the parameters of the experiment and all the physics are present in the code. But the closeness of our results to the experiment at the end, suggests we are very close to achieving this goal.

There are also considerations about the speed of our simulations. WAKE with QSA approximation is a very fast and reliable code. On the other hand the promotion process has some drawbacks. As we promote more and more particles and solve the exact equations the software slows down considerably. Comparing two cases of $\gamma_{t}=5$ and $\gamma_{t}=1.1$ shows for the former case it takes about 14 hours to simulate $1.4 \mathrm{~cm}$ propagation in the capillary on a single desktop CPU whereas for $\gamma_{t}=1.1$ it takes nearly 18 hours to run the simulation on the same CPU. Still it is orders of magnitude faster than codes like 
OSIRIS and others and can be used on a single machine to do simulations and get results with remarkable accuracy. 


\subsection{Figures}

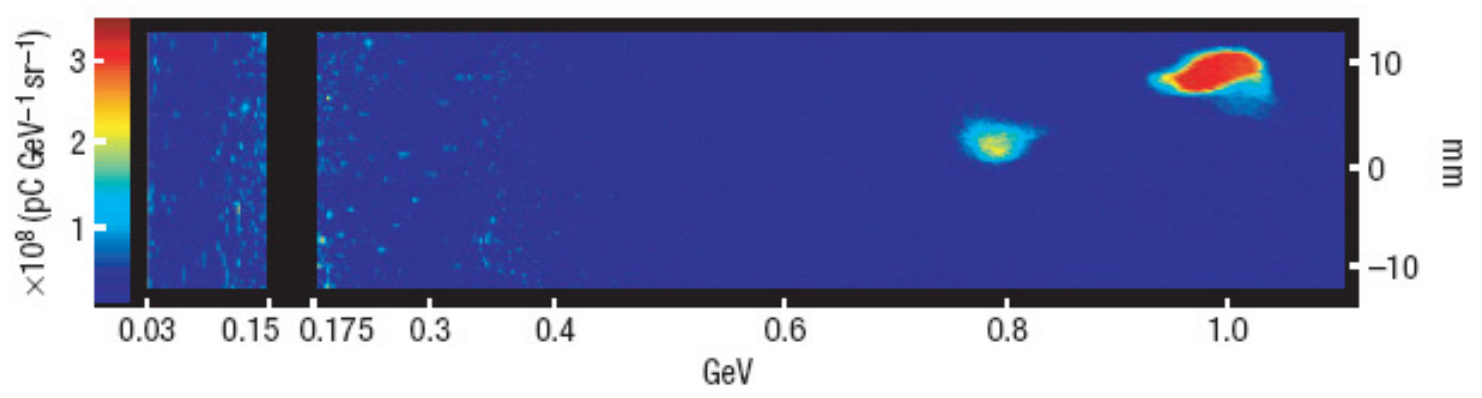

Fig.4.1) Leemans et al. [3] results for 40TW laser. About 30pc of electrons are accelerated to $1 \mathrm{GeV}$. The horizontal axis is beam energy and the vertical axis is the beam size. 


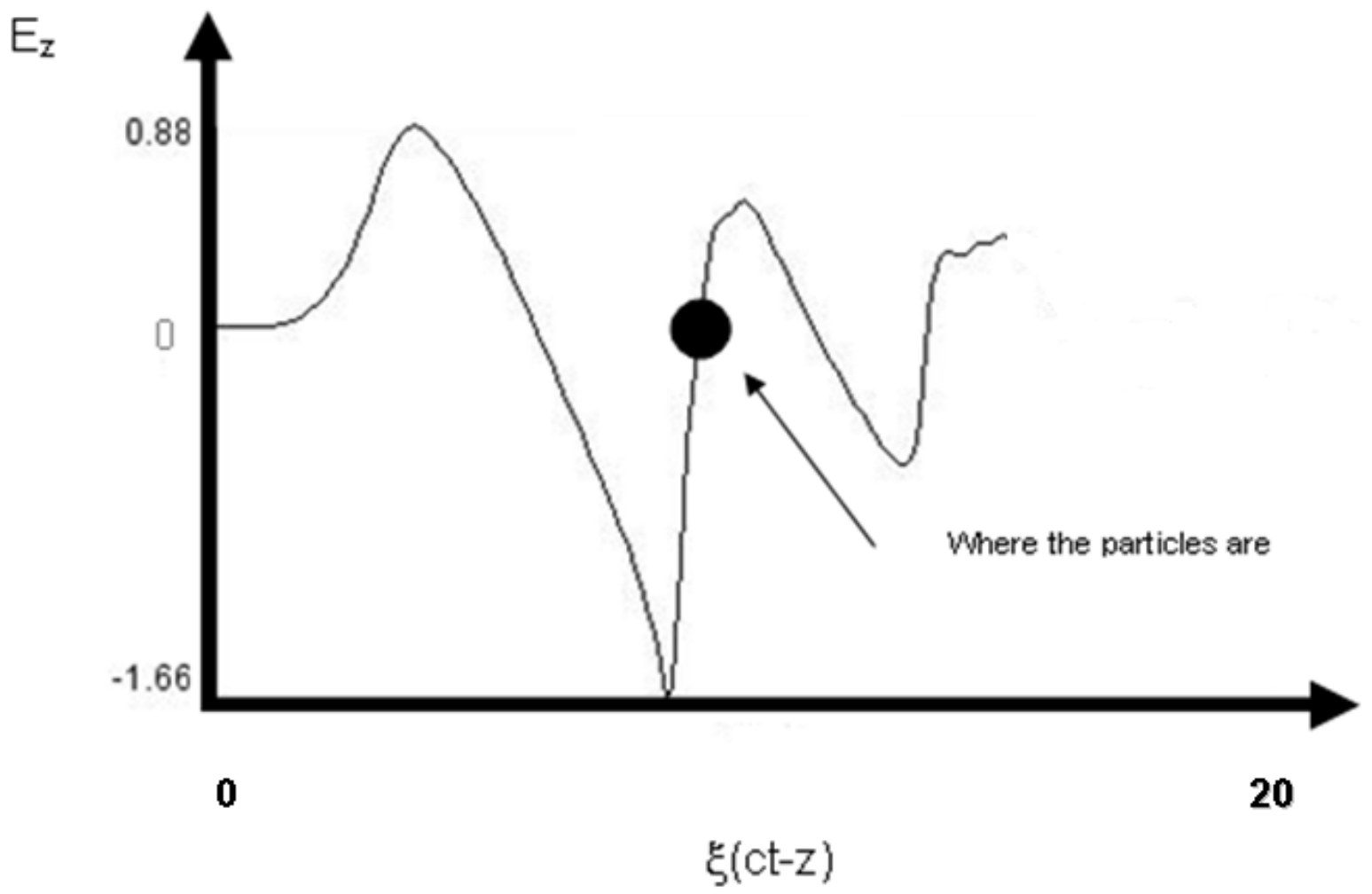

Fig. 4.2) The placement of particles in the wakefield created by laser, in the case of externally injected particles. 


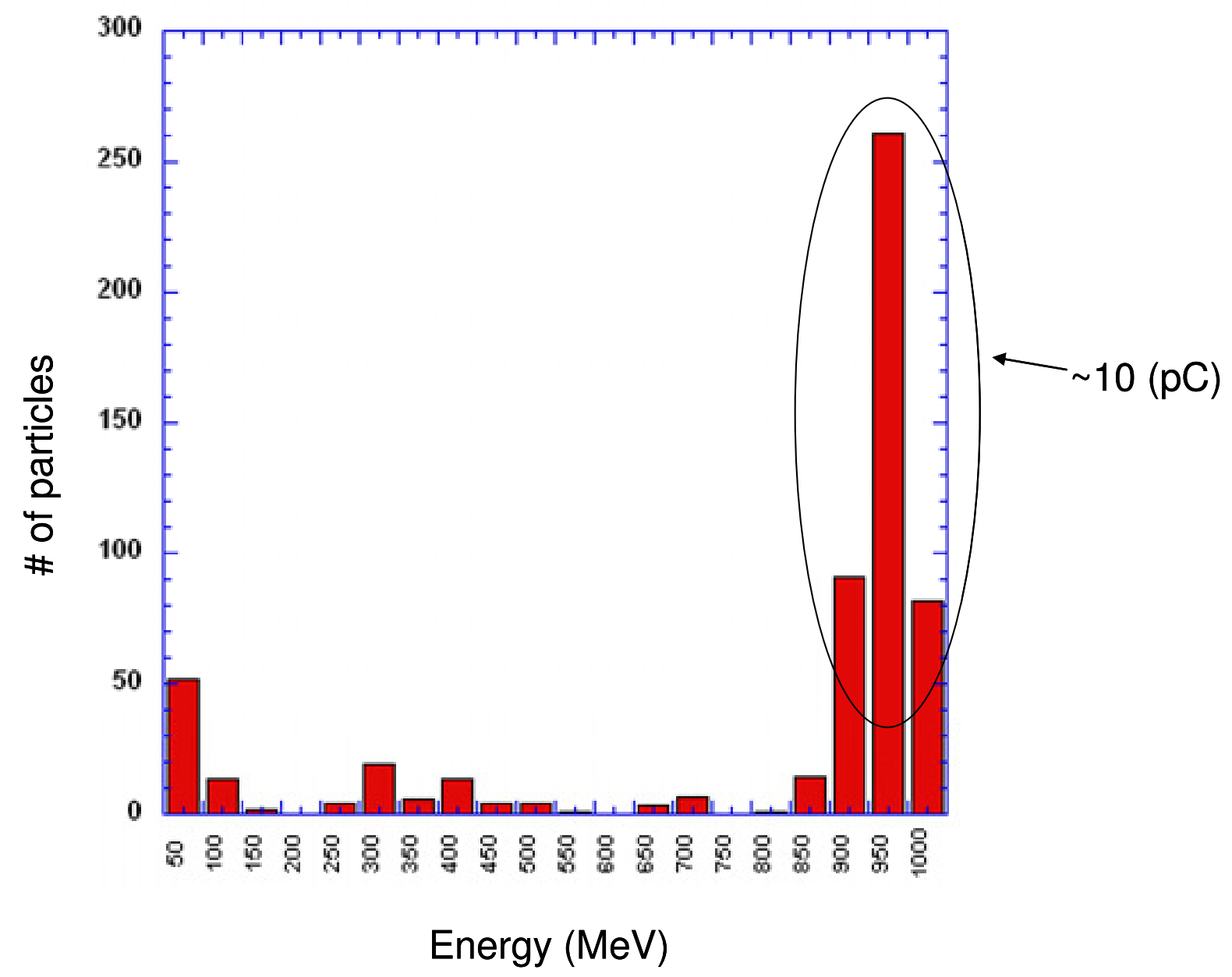

Fig.4.3) Histogram of energy of the externally injected particles after $1.4 \mathrm{~cm}$ propagation in the channel. 


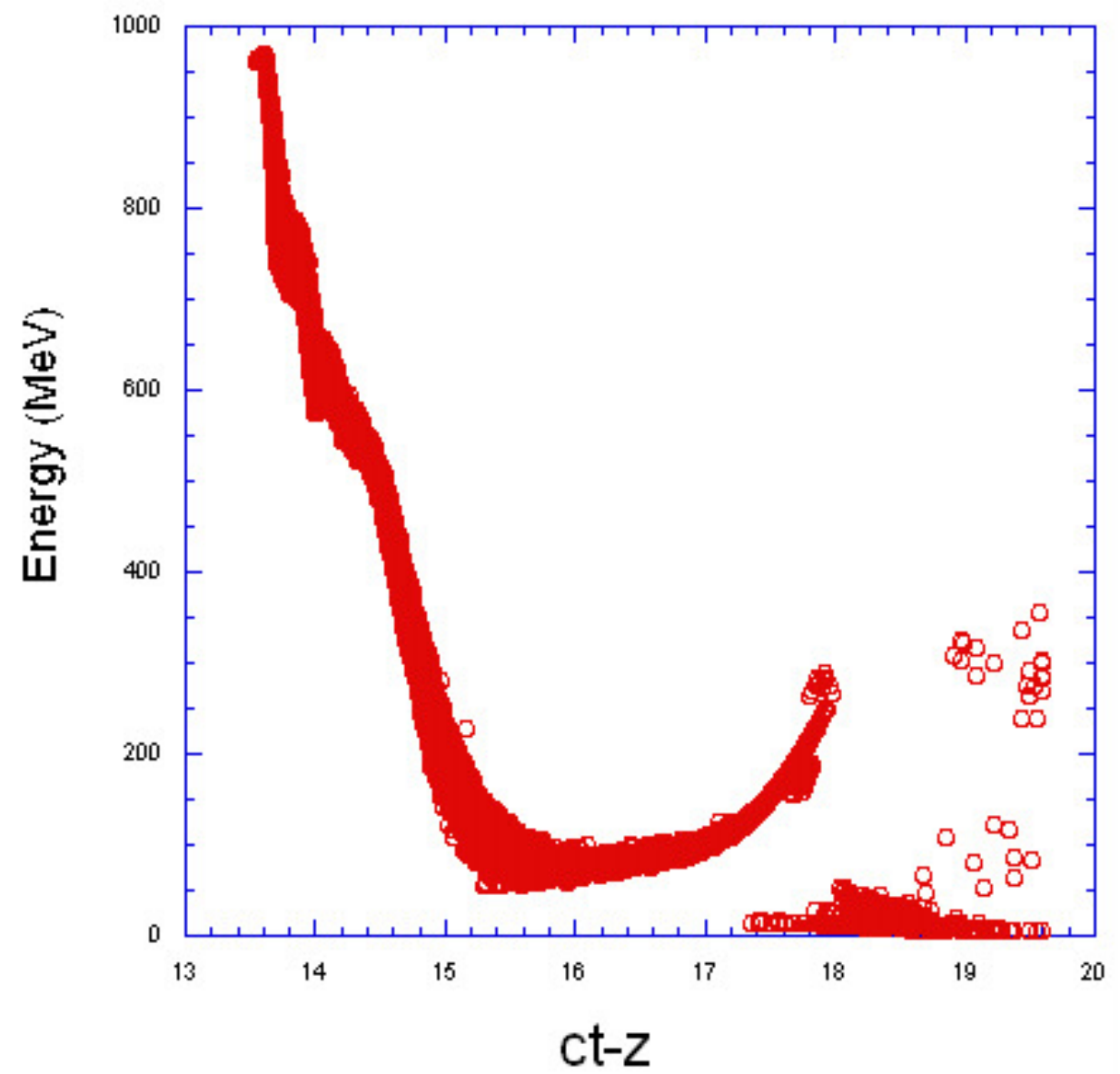

Fig. 4.4) Energy distribution of particles in $\xi$ with $\gamma_{t}=2.5$ when only threshold trapping is implemented. 


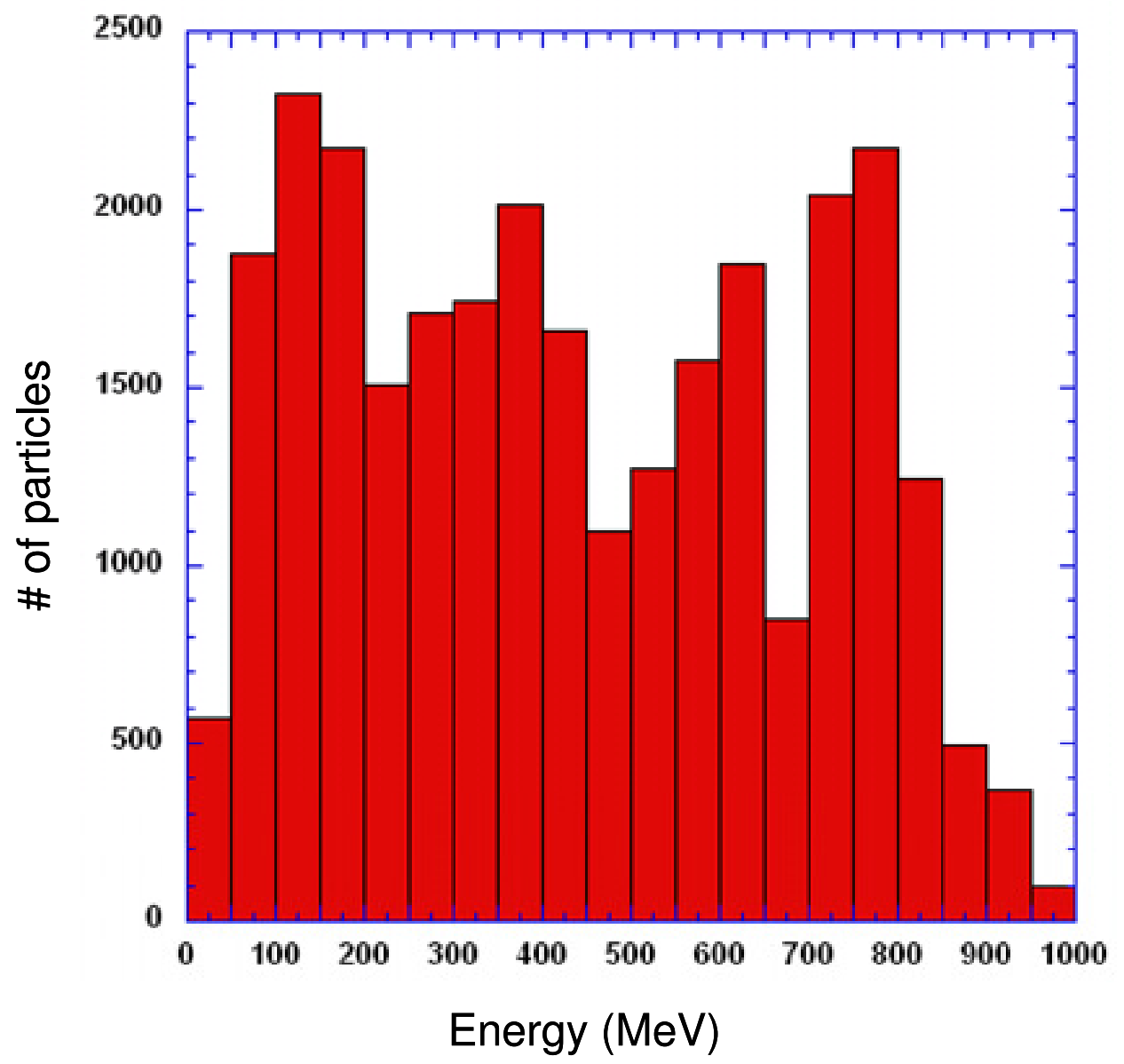

Fig. 4.5) Histogram of energy of beam particles in the case of $\gamma_{t}=2.5$ 


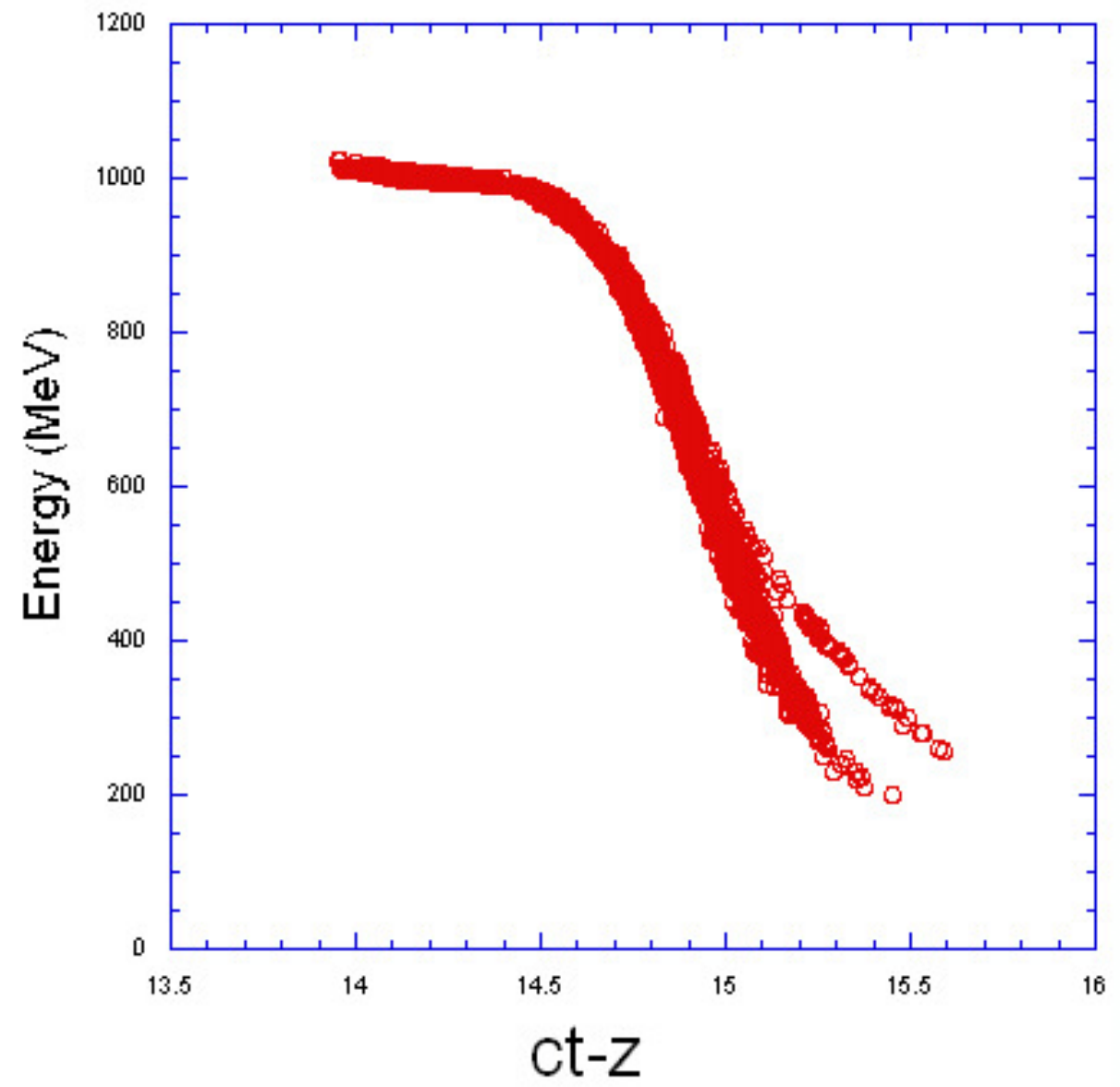

Fig. 4.6) Energy distribution of particles in $\xi$ with $\gamma_{t}=4.5$ when only threshold trapping is implemented. 


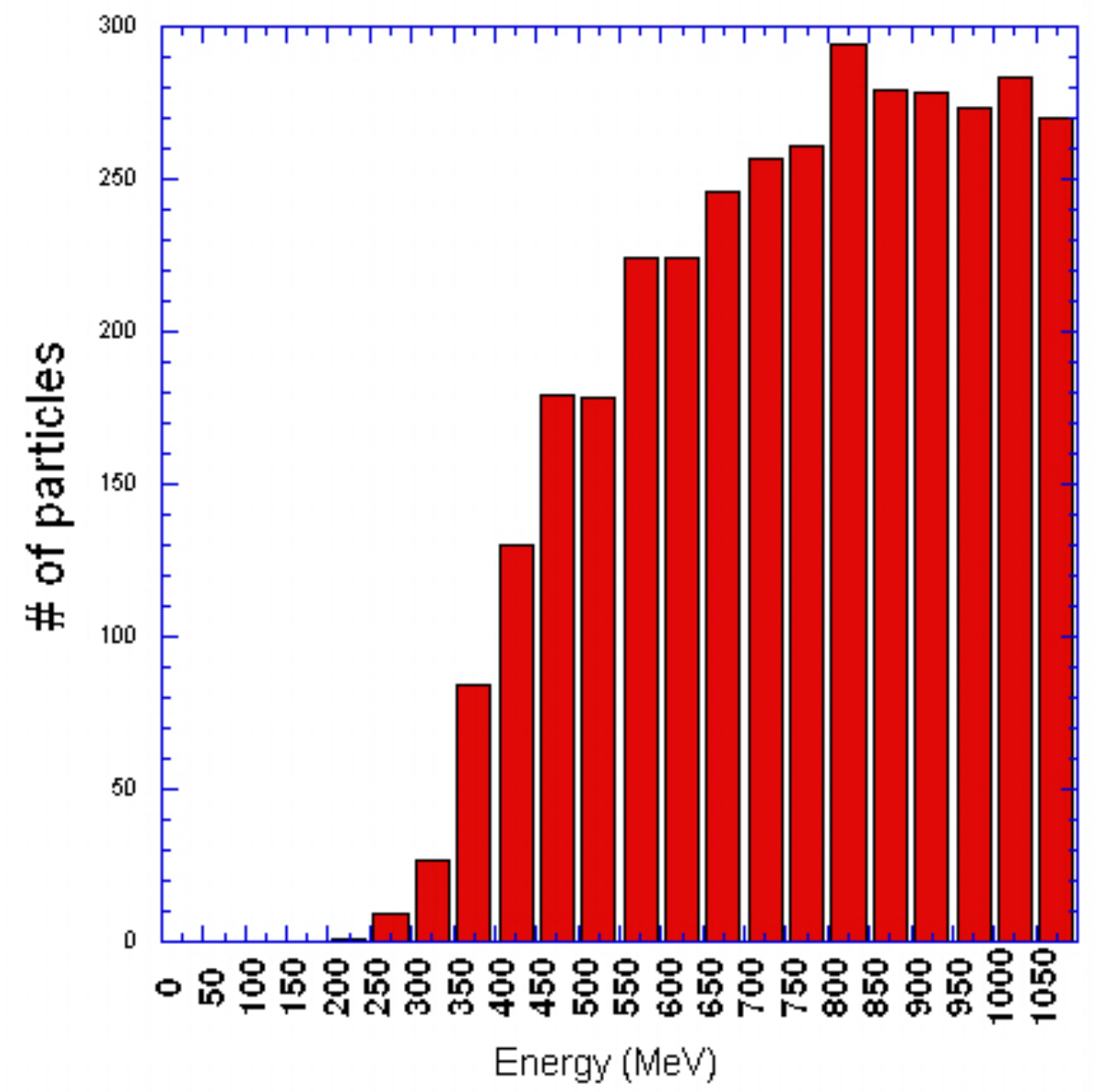

Fig. 4.7) Histogram of energy of beam particles in the case of $\gamma_{t}=4.5$ 

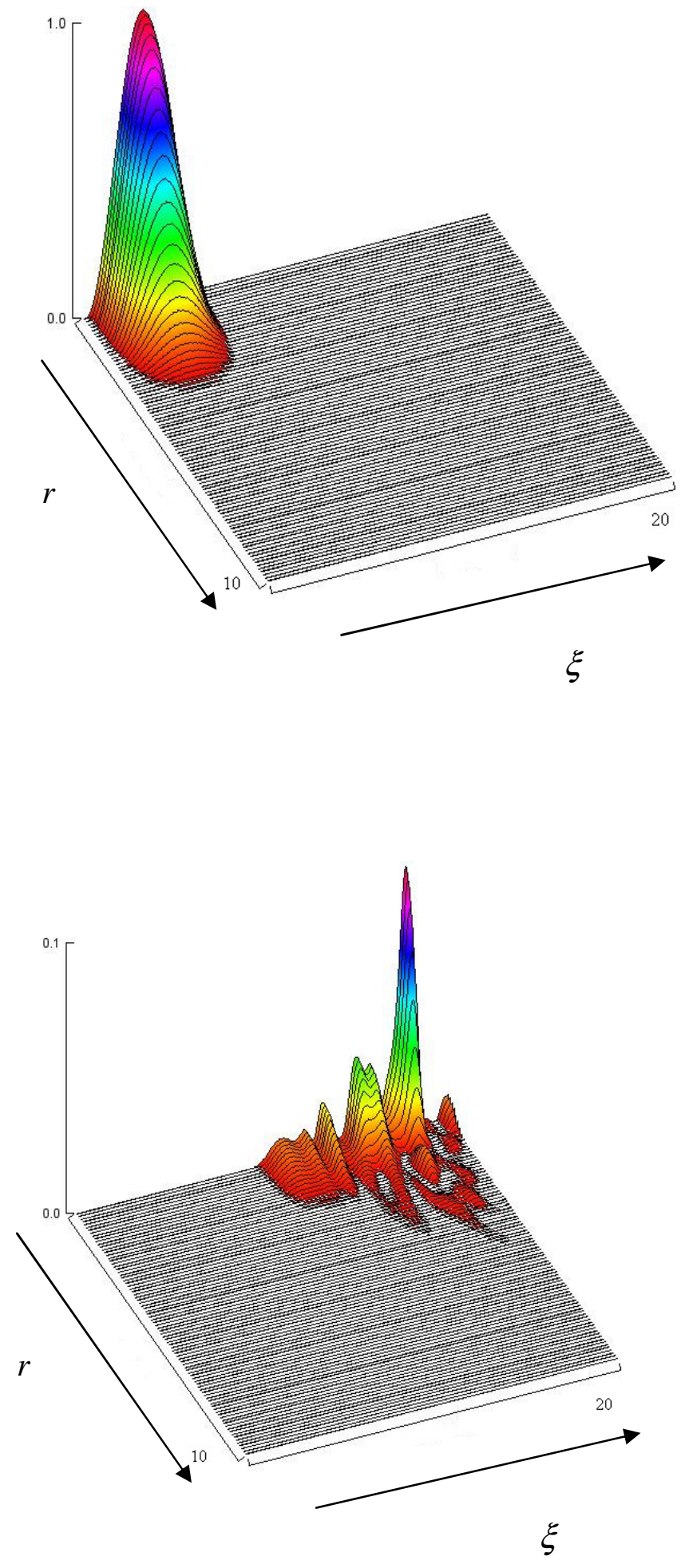

Fig. 3.8 (a) Laser intensity as it is initialized at the beginning of the simulation
Fig. 3.8 (b) Laser intensity at the end of simulation after $3.3 \mathrm{~cm}$ propagation in the channel. 


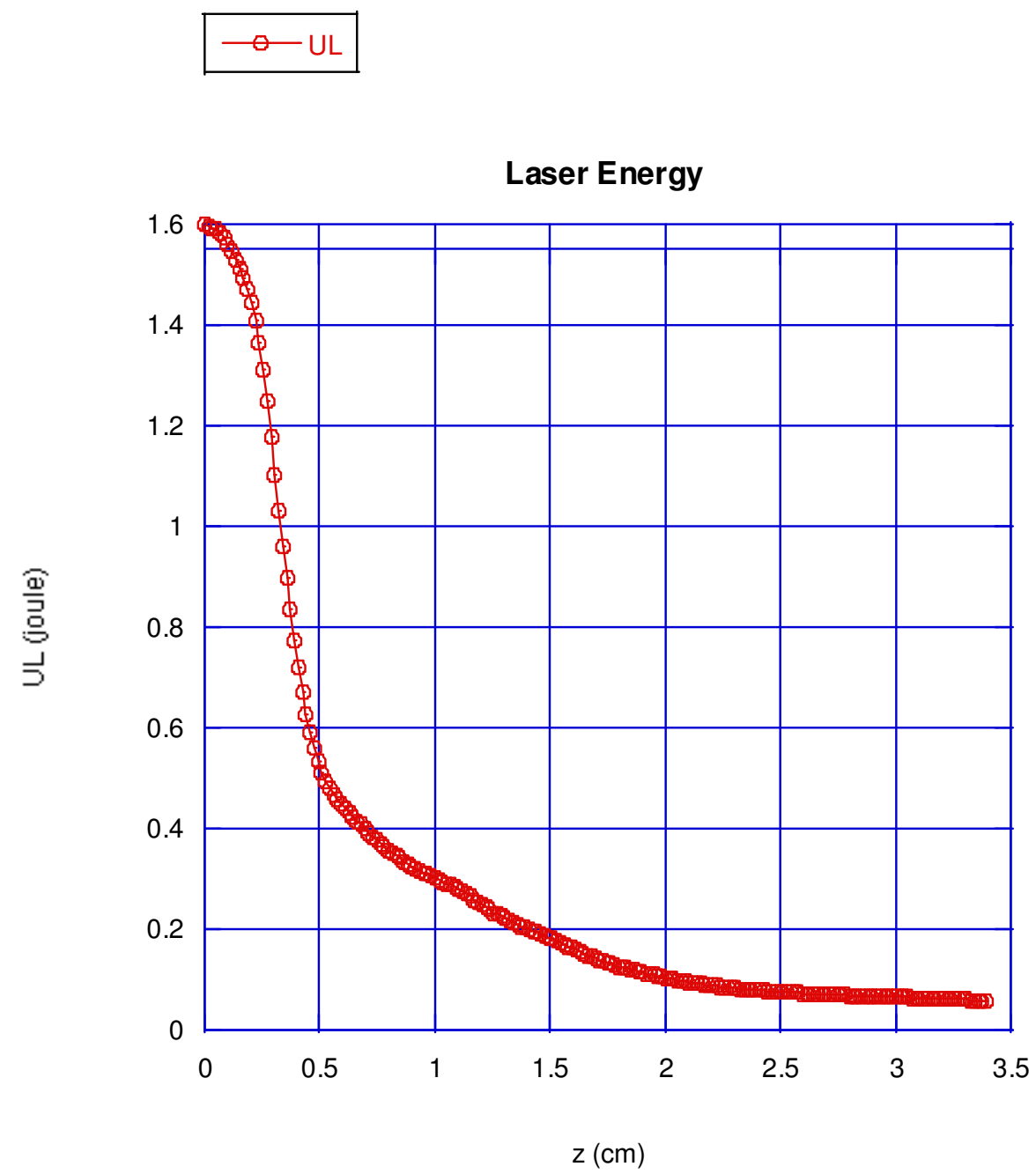

Fig. 4.9) Absorption of laser energy (in units of Joule) as it propagates in the channel. 


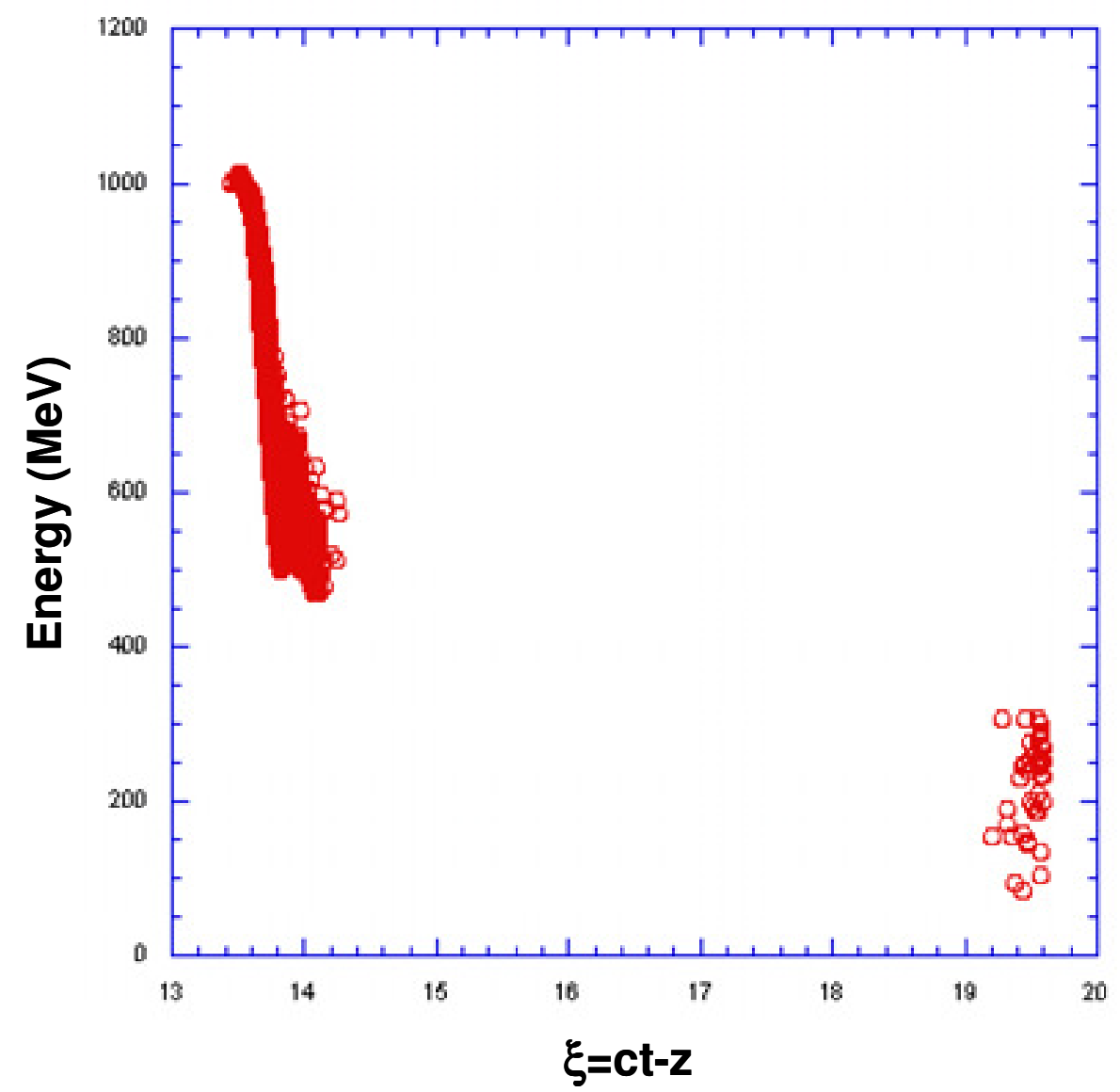

Fig. 4.10) Energy distribution of particles in $\xi$ with $\gamma_{t}=1.2$ in the LNBL experiment simulation with complete set of promotion conditions. 


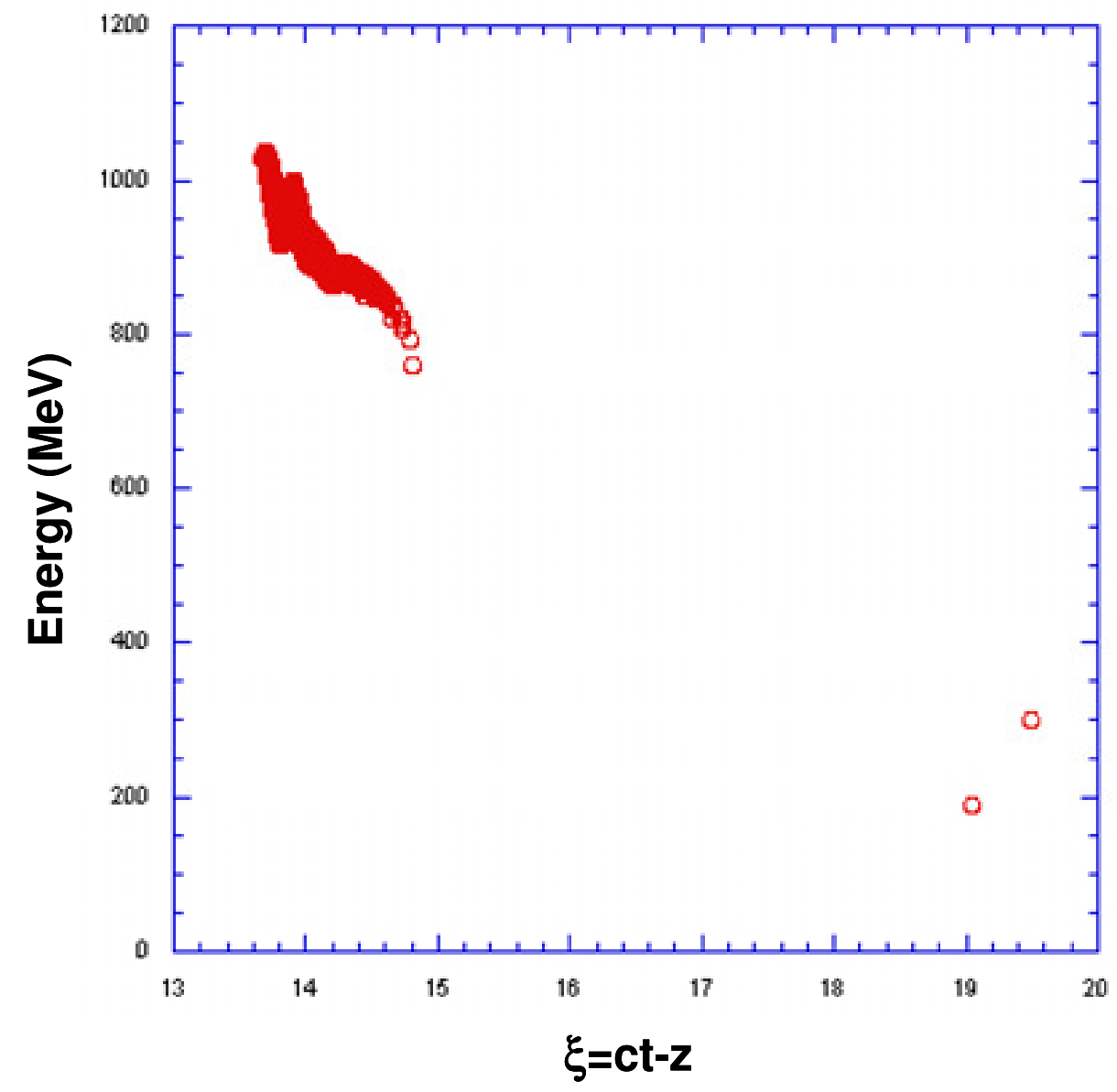

Fig. 4.11) Energy distribution of particles in $\xi$ with $\gamma_{t}=2.5$ in the LNBL experiment simulation with complete set of promotion conditions. 


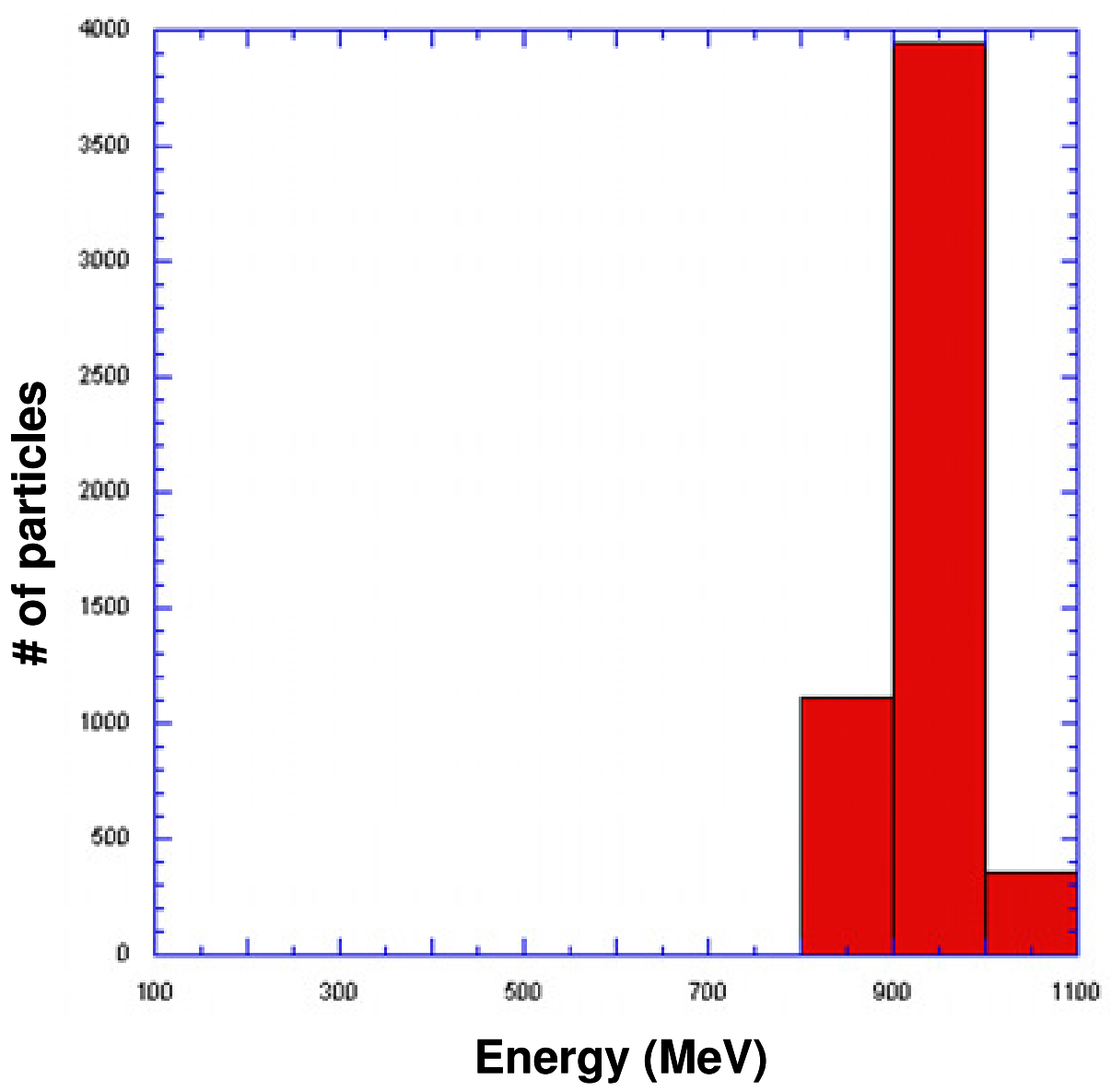

Fig. 4.12) Histogram of energy of beam particles in the case of $\gamma_{t}=2.5$. The graph shows very good agreement with the experimental data. 


\section{Chapter 5: Conclusion and Summary}

We have shown in this thesis the development of the code WAKE for simulating both PWFA and LWFA schemes. The Code has been modified from its original capability of modeling the propagation of short pulse lasers into under dense plasma to be able to simulate the self-trapping of plasma electrons.

To create a new code which has capability of dealing with energetic particles, one needs to overcome the assumption of QSA in code, which assumes that the driver and its wakefields are undisturbed during the transit time of plasma electrons. This however does not hold for energetic particles. The solution came about by defining two sets of particles, the plasma particles for which the QSA is valid, and the beam particles, which travel in the driver frame. In order to transit from plasma particle to beam particle status a set of promotion conditions were proposed. These conditions are, monitoring the radial position of particles and also having an energy threshold.

We presented theoretical work to estimate the range of values for the energy threshold. Also simulations were performed for different values of threshold to better understand the process. At the end it was shown lowering the threshold to the smallest possible value is the most physical assumption we can have. But this causes a large number of plasma particles to be promoted to beam status. It was noted the increased number of promoted particles to the beam status, and removing them from background plasma, consequently produces some amount of noise in the data which accumulates over long time, and had to be overcome. So a complementary method of promoting the particles to beam status has been developed. In this method one follows the trajectory of 
plasma particles in addition to their energy threshold and when a particle comes close to the axis (wave breaking) then it is promoted to beam status. This was confirmed by observation of trajectories of particles which are trapped in the wakefield. The outcome showed less unnecessary promotion (nearly by a factor of 4) and much faster and smoother simulations.

The results have been compared with experimental results and also full PIC code simulations, which showed good agreement with the maximum energy gained in the simulations from WAKE but with slightly less desirable energy spread. One can attribute the differences to the unimplemented and also unknown dynamics of the experimental results or as mentioned before some level of noise in the system.

The code WAKE is a very useful tool to get fast results with very high accuracy on a single desktop machine to explore different methods of acceleration before exploring much more expensive method of going into doing full 3D PIC codes on cluster of processors for days and even weeks. All the results in this thesis have been obtained on simulations which took no longer than one day on a single processor desktop computer. 


\section{Bibliography}

[1] M. Tinger “Does Accelerator-Based Particle Physics Have a Future?” Physics Today, vol.54, No. 1 (2001).

[2] P. Mora and T. Antonsen "Kinetic modeling of intense, short laser pulses propagating in tenuous plasmas” Phys. Plasmas 4, pp217-229 (1997).

[3] W. P. Leemans, et al. "GeV electron beams from a centimetre-scale accelerator" nature physics vol 2 ( 2006).

[4] W. Lu et al. "Generating multi GeV electron bunches using single stage laser wakefield acceleration in a 3D nonlinear regime” Phys. Rev. ST Accel. Beams 10, $061301(2007)$.

[5] E. Esarey et al. "Optically guided laser wake-field acceleration” Phys. Fluids B Vol.5 (7), pp 2690-2697, (1993).

[6] P. Sprangle and E. Esarey "Interaction of ultrahigh laser fields with beams and plasmas” Phys. Fluids B Vol.4 (7), pp 2241-2248, (1992).

[7] P. Sprangle et al. "Propagation and guiding of intense laser pulses in plasmas" PRL, Vol. 69 (15), (1992).

[8] K. Krushelnick et al. "Laser plasma acceleration of electrons: Towards the production of monoenergetic beams” Phys. Of Plasmas, Vol. 12, 056711 (2005).

[9] C. Joshi "Review of Beam Driven Plasma Wakefield Accelerators" Advance accelerator concepts, $11^{\text {th }}$ workshop, (2004).

[10] P. Muggli et al. “Meter-Scale Plasma-Wakefield Accelerator Driven by a Matched Electron Beam” PRL, Vol. 93 (1), (2004). 
[11] M.J. Hogan et al. "Multi-GeV Energy Gain in a Plasma-Wakefield Accelerator" PRL, Vol. 95, 054802, (2005).

[12] E. Kallos et al. "High-Gradient Plasma-Wakefield Acceleration with Two Subpicosecond Electron Bunches” PRL, Vol. 100, 074802, (2008).

[13] Tajima, T. \& Dawson, J. M.” Laser electron accelerator" Phys. Rev. Lett. 43, pp267-270 (1979).

[14] C. Coverdale et al. "Electron acceleration from the breaking of relativistic plasma waves" Nature, vol. 337, pp. 606-6-8, (1995).

[15] J. M. Dawson, "Nonlinear electron oscillations in a cold plasma" Phys. Rev., vol. 133, pp. 383-387, (1959).

[16] E. Esarey et al. "Overview of Plasma-based Accelerator Concepts" IEEE Trans. Plasma Science, 24, pp 252-288 (1996).

[17] C. Huang et al. "QUICKPIC: A highly efficient particle-in-cell code for 3 modeling wakefield acceleration in plasmas" J. computational Physics 217, pp.658-679, (2006).

[18] J. B. Rosenzweig et al. "Experimental measurement of nonlinear plasma wakefields," Phys. Rev. A, vol. 39, pp. 1586-1589, (1989).

[19] P. Chen et al. "Acceleration of electrons by the interaction of a bunched electron beam with a plasma," Phys. Rev. Lett., vol. 54, pp. 693-696, (1985).

[20] K. Nakajima et al. "Proof-of-principle experiments of laser wakefield acceleration using a 1 ps 10 TW Nd:glass laser” AIP Advanced Accelerator Concepts, vol. 335 Ed. New York: Amer. Inst. Phys., pp. 145-155, (1995).

[21] P. Sprangle et al. "Laser wakefield acceleration and relativistic optical guiding," Appl. Phys. Lett., vol. 53, pp. 2146-2148, (1988). 
[22] T. Katsouleas et al. "Laser wakefield acceleration with highly relativistic pumps” in AIP Advanced Accelerator Concepts, vol. 193, C. Joshi, Ed. New York: Amer. Inst. Phys., pp. 165-171, (1989).

[23] C.G.R. Geddes et al. "High-quality electron beams from a laser wakefield accelerator using plasma-channel guiding” Nature, Vol. 431, (2004).

[24] S.P.D. Mangles et al. "Monoenergetic beams of relativistic electrons from intense laser-plasma interactions” Nature, Vol. 431, (2004).

[25] J. Faure et al "A laser-plasma accelerator producing monoenergetic electron beams” Nature, Vol. 431, (2004).

[26] T. Katsouleas “Electrons hang ten on laser wake” Nature, Vol. 431, (2004).

[27] Y. Kitagawa et al. "Beat-wave excitation of plasma wave and observation of accelerated electrons” Phys. Rev. Lett., Vol. 68, pp. 48-51, (1992).

[28] K. Nakajima et al. “Observation of ultrahigh gradient electron acceleration by a self-modulated intense short laser pulse” Phys. Rev. Lett., Vol. 74, pp. 44284431, (1995).

[29] E. Esarey et al. "Synchrotron radiation from electron beams in plasma-focusing channels” PRE, Vol. 65, 056505, (2002).

[30] R. Bingham et al. "Plasma based charged-particle accelerators" Plasma Phys. Control. Fusion, Vol. 46 (2004).

[31] J. R. Cary and C. L. Bohn "Computational Accelerator Physics Working Group Summary" Advance accelerator concepts, $11^{\text {th }}$ workshop, (2004).

[32] T.M. Antonsen and P. Mora "Self-focusing and Raman scattering of laser pulses in tenuous plasmas” Phys. Fluids B, Vol.5 (5), (1993). 
[33] S. V. Bulanov et al. "Nonlinear depletion of ultrashort and relativistically strong laser pulses in an underdense plasma" Phys. Fluids B Vol.4 (7), pp 1935-1942, (1992).

[34] D.J. Spence et al. "Gas-filled capillary discharge waveguides" J. Opt. Soc. Am. B, Vol. 20 (1), (2003).

[35] C. Joshi "Laser Plasma Accelerators: a status report" Advance accelerator concepts, $9^{\text {th }}$ workshop, (2001).

[36] S. Wilks et al. "Beam Loading Efficiency in Plasma Accelerators" IEEE, PAC, pp100-102, (1987).

[37] R.P. Sharma et al "Effect of relativistic and ponderomotive nonlinearities on stimulated Raman scattering in laser plasma interaction" Phys. Of Plasmas, Vol. 13, 113109 (2006).

[38] D. Umstadter et al. "Laser Injection of Ultrashort Electron Pulses into Wakefield Plasma Waves” PRL, Vol. 76 (12), (1996).

[39] W.B. Mori "Advances in simulation capability: A path towards modeling 10-100 GeV plasma accelerator stages" Advance accelerator concepts, $11^{\text {th }}$ workshop, (2004).

[40] N. Hafz et al. "Numerical Simulation for Plasma Electron Acceleration by $12 T W 50$ Fs Laser Pulse” Advance accelerator concepts, $9^{\text {th }}$ workshop, (2001).

[41] L.M. Garbunov et al. "Laser wakefield acceleration by petawatt ultrashort laser pulses” Phys. Of Plasmas, Vol. 12, 033101 (2005).

[42] P. Sprangle et al. "Stable Laser-Pulse Propagation in Plasma Channels for GeV Electron Acceleration” PRL, Vol. 85 (24), (2000).

[43] P. Sprangle et al. "Nonlinear interaction of intense laser pulses in plasma" Phys. Rev. A, Vol. 41 (8), (1990). 
[44] S.Y. Kalmykov et al. "Injection, trapping, and acceleration of electrons in a threedimensional nonlinear laser wakefield” Phys. Of Plasmas, Vol. 13, 113102, (2006).

[45] R.F. Hubbard et al. "Scaling of Accelerating Gradients and Dephasing Effects in Channel-Guided Laser Wakefield Accelerators" IEEE Transactions on Plasma Science, Vol. 28 (4), (2000).

[46] J. Palastro et al. "Pulse propagation and electron acceleration in a corrugated plasma channel” PRE Vol. 77, 036405, (2008).

[47] I. Kostyukov " Phenomenological theory of laser-plasma interaction in "bubble” regime” Phys. Plasmas, Vol. 11 (11), pp.5256-5264, (2004).

[48] Y. Ehrlich et al. "Guiding of High Intensity Laser Pulses in Straight and Curved Plasma Channel Experiments” Phys. Rev. Lett. 77, 4186 - 4189 (1996).

[49] S. Jackel et al. "Channeling of terawatt laser pulses by use of hollow waveguides" Opt. Lett. 20, 1086, (1995).

[50] C. G. Durfee and H. M. Milchberg. "Light pipe for high intensity laser pulses" Phys. Rev. Lett. 71, 2409 - 2412 (1993).

[51] W. Leemans et al. “Laser guiding for GeV laser-plasma Accelerators” Phil. Trans. R. Soc. A 364, pp585-600, (2006).

[52] J. Fuchs et al. "Laser-driven proton scaling laws and new paths towards energy increase” Nature Phys. Vol. 2, (2006).

[53] N.H. Matlis et al. "Snapshots of laser wakefields” Nature Phys. Vol. 2, (2006).

[54] J.V. Tilborg et al. "Terahertz radiation as a bunch diagnostic for laser-wakefieldaccelerated electron bunches” Phys. Of Plasmas, Vol. 13, 056704, (2006). 
[55] P. Michel et al. "Radiative damping and electron beam dynamics in plasma-based accelerators" PRE, Vol. 74, 026501, (2006).

[56] P. Sprangle et al. "Nonlinear theory of intense laser-plasma interaction" PRL, Vol. 65 (17), (1990).

[57] S. Gordienko et al. "Scalings for ultrarelativistic laser plasmas and quasimonoenergetic Electrons" Phys. Of Plasmas, Vol. 12, 043109, (2005).

[58] W. Lu et al. "A nonlinear theory for multidimensional relativistic plasma wave wakefields" Phys. Of Plasmas, Vol. 13, 056709, (2006).

[59] S. Wang et al. "X-Ray Emission from Betatron Motion in a Plasma Wiggler" PRL, Vol. 88 (13), (2002).

[60] D. Whittum et al. "Electron Hose-Instability in the Ion-Focused regime" PRL, Vol. 67 (8), (1991).

[61] J. Osterhoff et al. "Generation of Stable, Low-Divergence Electron Beams by LaserWakefield Acceleration in a Steady-State-Flow Gas Cell" PRL, Vol. 101, 085002, (2008).

[62] V. Malka, et al. "Electron Acceleration by a Wake Field Forced by an Intense Ultrashort Laser Pulse” Science 298, 1596 (2002).

[63] A. Pukhov et al. "Laser wake field acceleration: the highly non-linear broken-wave regime” Appl. Phys. B 74, pp355-361 (2002).

[64] A. Ting et al. "Nonlinear Wakefield Generation and Relativistic focusing of Intense Laser Pulses in Plasmas" Phys. Fluids B, Vol.2 (6), (1990).

[65] H.M.Milchberg et al. "Development and applications of a plasma waveguide for intense laser pulses”. Physics of Plasmas, Vol. 3(5): pp2149-2155, (1996). 
[66] G. Mourou “The ultrahigh-peak-power laser: Present and future” Applied Physics B-Lasers and Optics” Vol. 65(2): pp 205-211, (1997).

[67] J.R. Penano et al. "Raman forward scattering and self-modulation of laser pulses in tapered plasma channels” Physical Review E, Vol. 66(3), (2002).

[68] C.E. Max et al. "Self-Modulation and Self-Focusing of Electromagnetic-Waves in Plasmas” PRL ,Vol. 33(4), pp209-212, (1974).

[69] Gordon et al. "A Ponderomotive Guiding Center Particle-in-Cell Code for Efficient Modeling of Laser-Plasma Interactions, IEEE Transactions on Plasma Science, Vol. 28(4), pp1224-1232, (2000).

[70] A. Solodov. et al. "Simulation of photon acceleration in a plasma wake” Phys. Of Plasmas, Vol. 6 (503), (1999).

[71] A. Solodov "Interaction of ultra short, ultra intense laser pulse with underdense plasma” Dissertation thesis, Ecole Polytechnique (2000).

[72] R.A. Fonseca et al "OSIRIS: A three-dimensional, fully relativistic particle-in-cell code for modeling plasma based accelerators" Computational Science, pp. 342-351, (2002). 
